(Aus dem pharmakologischen Institut der Cniversität Zürich.)

\title{
Über die Wirkung der Schilddrüse auf den Blutkreislauf.
}

\author{
Von
}

\section{Adolr Oswald.}

(Mit 40 Textfiguren.)

Die Wirkung der Schilddrüse auf den Blutkreislauf ist in den letzten 20 Jahren vielfach Gegenstand der Untersuchung gewesen. Die erreichten positiven Resultate stehen aber in keinem Verhältnis zum Umfang der darauf verwendeten Arbeit, denn tatsächlich herrscht. sozusagen noch in keinem einzigen Punkte Einigkeit unter den Experimentatoren. Während die einen bei intravenöser Zufuhr von Schilddrüsenprodukten eine Wirkung auf den Blutdruck fanden, wird sie von anderen in Abrede gestellt, oder die einen fanden eine Drucksteigerung und die anderen eine Erniedrigung. Die einen konstatierten Pulsbeschleunigung, die anderen Verlangsamung, andere wiederum keine Veränderung des Rhythmus. Die einen stellten eine fördernde Wirkung anf die Ansprechbarkeit des Vagus und des Depressor fest und die anderen verneinten jede Beeinflussung nach dieser Richtung, oder aber man sprach von einer nicht spezifischen, vom Jodgehalt abhängigen Wirkung, die auch mit anderen jodhaltigen Eiweissstoffen oder -produkten erreichbar sei. Eine befriedigende Erklärung für diese Divergenzen ist bisher nicht zu finden.

Durchgeht man aufmerksam die Literatur, so wird man allerdings alsbald gewahr, dass durchaus nicht alle Arbeiten untereinander vergleichbar sind. Während die einen Experimentatoren Press saft aus der Schilddrüse oder wässerige Extrakte (mit oder ohne Glyzerinzusatz) der frischen oder getrockneten Drüse verwandten, gebrauchten andere das Baumann'sche Jod othyrin. Weder die einen noch die anderen können aber ein endgültiges Resultat liefern. Denn in den Presssaft sowie in ein Vollextrakt gehen infolge Zer- 
malmung des Drüsenkörpers Substanzen über, die in vivo nicht oder wenigstens nicht in der gleichen Menge in den Säftestrom gelangen, so dass Effekte ausgelöst werden können, die im lebenden Organismus gar nicht zustandekommen. Andererseits kommt das Jodothyrin als solches in der Schilddrüse nicht vor, sondern stellt ein künstliches Spaltungs- und teilweise auch Umwandlungsprodukt des eigentlichen Schilddrüsensekretes, des Jodthyreoglobulins ${ }^{1}$ ) dar. Es kann daher ebensogut physiologische Eigenschaften besitzen, die seiner Muttersubstanz nicht zukommen, wie auch solche eingebüsst haben, die dieser anhaften. Das letztere ist in der Tat iu einer gewissen Beziehung nachgewiesen. So konnten Pick und Pineles ${ }^{2}$ ) ihre experimentell myxödematös gemachten Zicklein mit Jodothyrin nicht heilen, wohl aber mit Jodthyreoglobulin. Untersuchungen, die mit Jodothyrin angestellt sind, sagen somit nichts aus über das genuine Schilddrüsensekret. Den Physiologen und auch Pathologen interessiert aber das Drüsensekret, nicht ein künstliches, im Organismns nicht vorkommendes Spaltungsund Umwandlungsprodukt desselben. Trotzdem nun aber das Jodthyreoglobulin uns jetzt schon seit 16 Jahren bekannt ist, werden die experimentellen Untersuchungen immer noch mit dem Baumann'schen Jodothyrin angestellt, und die damit gewonnenen Resultate übertönen die wenigen mit Jodthyreoglobulin erbobenen Befunde; ist man doch nur zu rasch bei der Hand, Beobachtungsresultate vom Jodothyrin auf das Jodthyreoglobulin ohne weiteres zu übertragen.

Ein zweiter Punkt, der beim Studium der Literatur auffällt, ist, dass für die Entscheidung der Frage der Wirksamkeit der Schilddrüse gegenüber den deu Blutdruck und den Herzrhythmus beeinflussenden Nerven ganz verschiedenartige Methoden angewandt wurden, deren Gleichwertigkeit gar nicht erwiesen war.

Endlich will ich gleich vorwegnehmen, dass sich aus meinen Untersuchungen ergeben hat, dass bei den Versuchstieren individuelle und auch Rassenunterschiede bestehen, welche ein abweichendes Verhalten bedingen. Hierauf sind, wie wir sehen werden, manche unter-

1) Ad. Oswald, Die Eiweisskörper der Schilddrüse. Zeitschr. f. physiol. Chemie Bd. 27 S. 14.1899.

2) E. P. Pick und F. Pineles, Untersuchungen über die physiologisch wirksame Substanz der Schilddrüse. Zeitschr. f. exp. Path. u. Therap. Bd. 7 S. 518.1909. 
schiedliche Befunde und in gewisser Hinsicht entgegengesetzt lautende Resultate namentlich von Autoren, die nur eine Tierart oder Rasse zu ihren Versuchen verwendet haben, zurückzuführen.

Einen Modus besonderer Art schlugen die Arbeiten A'sher's und seiner Mitarbeiter ${ }^{1}$ ) ein, die die Wirkung des : Schilddrüsensekretes dadurch festzustellen suchten, dass sie die die Drüse versorgenden Nerven reizten und die danach beobachteten Effekte studierten. Wenn diese Untersuchungsart den Vorteil hat, die Verhältnisse im lebenden Körper möglichst nachzuahmen und durch Benützung der physiologischen Reizbahnen die Tätigkeit der Zelle nach Möglichkeit in der normalen Richtung zu belassen, somit die Absonderung eines möglichst phy siologische,n Sekretes zu gewährleisten, so ist damit nichts ausgesagt über die Natur dieses Sekretes, das heisst wir wissen nicht, welchem Bestandteil des Sekretes eine bestimmte Wirkung zuzuschreiben ist. Das Endziel der Physiologie wie der Pharmakologie ist aber, mit chemisch reinen Stoffen zu arbeiten und komplexe Wirksamkeiten in einzelne Glieder zu zerlegen. Diesem Postulat kann die Reizung eines Sekretionsnerven niemals genügen - so grossen Wert diese Methode auch nach anderer Richtung hat. Gleichviel will ich bemerken, dass die von Asher und seinen Mitarbeitern nach ihrer Methode gewonnenen Resultate mit den meinen übereinstimmen, was bei der grossen Verwirrung, die in der Schilddrüsenliteratur herrscht, gewiss als erfreulich zu bezeichnen ist.

Auf die Historik der Frage will ich nicht eingehen. Sie ist in vielen Abhandlungen und zusammenfassenden Darstellungen eingehend gewürdigt. Ausserdem kommt es mir weniger auf eine geschichtliche Darlegung der Tatsachen an als auf eine kritische Abwägung des bisher Erreichten. Diese werde ich zweckmässig jeweilen der Mitteilung meiner eignen Beobachtungen folgen lassen.

\section{Herkunft und Herstellung des Versuchsmaterials.}

Das für meine Versuche verwendete Jodthyreoglobulin stammte aus Schilddrüsen von $\mathrm{Hammeln}$, Schweinen, Kälbern

1) L. A sher und M. Fla ck, Beiträge zur Physiologie der Drüsen. XVI. Mitteilung: Die innere Sekretion der Schilddrüse und die Bildung des inneren Sekretes unter dem Einfluss von Nervenreizung. Zeitschr. f. Biol. Bd. 55 S. 83. 1911. - L. A sher und W. v. Rodt, Die Wirkungen von Schilddrüsen- und Nebennierenprodukten und die sekretorische Innervation der Schilddrüse. Zentralbl. f. Physiol. Bd. 26 Nr. 5. 1912. 
und vom Menschen. Die Tierschilddrüsen erhielt ich aus dem hiesigen Schlachthause. Herrn Tierarzt Dr. Schellenberg bin ich für gewissenhafte Lieferung zu besonderem Danke verpflichtet, den ich auch hier abzustatten nicht versäumen möchte. Die Drüsen vom Menschen hatte ich von verschiedenen Kollegen und auch vom hiesigen Pathologischen Institut erhalten. Allen Herren, die durch bereitwilliges Entgegenkommen meine Untersuchungen förderten, sei an dieser Stelle mein aufrichtiger Dank ausgesprochen. Vom Mensehen wurden normale Schilddrüsen und Kröpfe verwendet und von letzteren solche mit und ohne Kropfherz- oder Basedowsymptome. Von Tieren kamen ausserdem Kolloidkröpfe und einfach hyperplastische, kolloidfreie Strumen zur Verwendung. Die Herstellung des Jodthyreoglobulins erfolgte nach dem von mir seinerzeit ausgearbeiteten Verfahren $^{1}$ ).

Da unter den Experimentatoren vielfach die Meinung zu herrschen scheint, die Herstellung sei mit besonderer Schwierigkeit verbunden - wenigstens erkläre ich mir nur so, dass auch noch bis $i^{n}$ die letzte Zeit hinein Drüsenvollextrakte oder Presssaft oder das käufliche Ba umann'sche Jodothyrin verwendet werden -, soll die Beschreibung des Verfahrens kurz wiederholt werden, wenn auch nur als Beleg dafür, dass es sehr einfach ist und auch dem chemisch weniger Geübten wenig Schwierigkeit bereiten dürfte.

Die zu Brei zerhackten Schilddrüsen werden mit Leitungswasser angerührt, ausgepresst und die Prozedur vier- bis fünfmal wiederholt. Die zusammengegossenen Auszüge werden mit der gleichen Menge kaltgesättigter Ammonsulfatlösung versetzt, der flockige Niederschlag nach einigen Stunden abfiltriert, in Wasser gelöst und nochmals in der gleichen Weise mit Ammonsulfat gefällt. Der abfiltrierte Niederschlag wird abermals in Wasser gelöst und die Lösung behufs Filtration auf mehrere Filter verteilt. Werden die erstmals durchgegangenen Filtrate mehrmals zurückgegossen, so fliesst alsdann eine wasserklare, wie Blutserum aussehende Lösung hindurch. Das klare Filtrat kann dann in zweifacher Weise weiterbehandelt werden: a) Es wird nochmals mit Ammonsulfatlösung in gleicher Weise wie vorher behandelt. Dabei entsteht ein schneeweisser, flockiger Niederschlag, der abfiltriert, in Wasser gelöst und die Lösung in Pergamentschläuchen gegen anfangs fliessendes, später destilliertes Wasser (bis

1) A. Oswald, l. c. 
zur Sulfatfreiheit des Dialysates) dialysiert wird. Diese Lösung dient zu den experimentellen Versuchen am Tier. Will man die Substanz als solche herstellen, so versetzt man die wässerige, salzfreie Lösung mit starkem Alkohol, filtriert nach einigen Stunden die ausgeschiedenen, zu Boden gesetzten weissen Flocken, trocknet und verreibt sie zu Pulver. Dieses kann beliebig lange aufbewahrt werden. b) Die Lösung wird mit sehr wenig verdünnter Essigsäure gefällt. Dabei entsteht ein weissflockiger Niederschlag, der abfiltriert und in mit wenig verdünnter Natronlauge versetztem Wasser gelöst wird. Diese Prozedur wird noch einmal wiederholt. Zuletzt wird abfiltriert und die Substanz getrocknet und zu Pulver zerrieben.

Das in Pulverform erhaltene Jodthyreoglobulin wird, wenn man es zu Versuchszwecken in Lösung braucht, mit schwach mit Natronlauge alkalisiertem Wasser versetzt (auf $1 \mathrm{~g}$ ca. $60 \mathrm{ccm}$ Wasser und etwa 5 Tropfen 10\% ige Natronlauge) und 1-2 Stunden in den Brutofen gestellt. Dabei resultiert eine braune Lösung, die man mit verdünnter Salzsäure möglichst neutralisiert, ohne dass es zur Fällung kommt. Ist zu viel Säure zugesetzt worden, so bringt man so viel pulverisiertes Natriumbikarbonat hinzu, bis die Lösung sich klärt.

Zur Aufbewahrung eignen sich auch die durch Ammonsulfat erhaltenen Flocken. Sie können in verschlossener Flasche unter entsprechend verdünnter Ammonsulfatlösung beliebig lange aufbewahrt werden. Zum Gebrauch können sie selbst nach jahrelangem Stehen abfiltriert, in Wasser gelöst und vom Ammonsulfat durch Dialyse befreit werden.

$\mathrm{Zu}$ meinen Versuchen wurde selbstdargestelltes, unter Ammonsulfat konserviertes oder in Pulverform trocken aufbewahrtes Jodthyreoglobulin verwendet, ferner ein Präparat, das ich der Liebenswürdigkeit der Farbenfabriken Fr. B a y er \& $\mathrm{Co}$. in Leverkusen bei Köln verdanke, welche mir ein grösseres Quantum für anderweitige Versuche hergestellt haben. Das B a u mann'sche Jodothyrin wurde mir ebenfalls freigebig von der gleichen Firma zur Verfügung gestellt, ebenso eine wasserlösliche Natronverbindung eines Jodothyrinpräparates. Ich spreche den Herren auch an dieser Stelle meinen Dank aus.

Es sei ausdrücklich noch darauf hingewiesen, dass dureh Dialyse die Präparate von eventuell mitgerissenen, wirksamen, kristalloiden Körpern befreit wurden und dass durch diesen Umstand der Einwand 
von vornherein beseitigt wurde, als sei nicht das Jodthyreoglobulin Träger der zu schildernden physiologischen Eigenschaften, sondern eine anhaftende kristalloide Substanz. Die Dialyse dauerte $3-5 \times 24$ Stunden gegen anfangs fliessendes, später destilliertes Wasser.

\section{Methodik.}

Als Versuchstiere wurden Kaninchen, Hunde und Katzen herangezogen. Zur Narkose wurde bei Kaninchen ausschliesslich Urethan verwendet, das den Tieren $1-1^{1 / 2}$ Stunden vor Beginn des Versuches in einer Menge von $1-2 \mathrm{~g}$, je nach der Grösse, in $25 \%$ iger Lösung unter die Rückenhaut beigebracht wurde. Die Hunde und Katzen erhielten Paraldehyd mit der Schlundsonde.

Da bei der. Präparierung der Halsnerven (bei Verwendung von Urethan) öfters hochgradige Cyanose eintritt, wurde bei allen Tieren und Tierarten die Tracheotomie vorgenommen und mittels einer tief in die Trachea eingeführten Glaskanüle Sauerstoff eingeleitet.

Die Narkose war durchweg sehr ruhig. Nur in ganz seltenen Fällen erfolgten Zuckungen. Diese Fälle wurden, wenn sie das Versuchsbild trübten, nicht verwendet. Einige wenige Male kam es vor, dass ohne ersichtlichen Grund plötzlich ein starker Tonusfall in den Gefässen eintrat. Auch diese Versuche wurden nicht verwendet.

Der Blutdruck wurde von der Carotis aus geschrieben. Als gerinnungshemmende Flüssigkeit wurde eine $28 \%$ ige Magnesiumsulfatlösung gebraucht. Die Zeichnung der Kurven auf dem berussten Kymographion geschah in der üblichen Weise unter Einschaltung eines Quecksilbermanometers. Als Sekundenschreiber wurde die Jaquet'sche Ubr verwendet.

Die Jodthyreoglobulinlösungen wurden bei den Kaninchen in eine Ohrvene injiziert, beim Hund und der Katze in die Vena jugularis oder femoralis. Was die übrigen Injektionen anbetrifft (Adrenalin, Suprarenin), so soll die Technik im betreffenden Abschnitt geschildert werden.

Zur Nervenreizung diente der durch ein mit einem Leclanchéelement gespeisten Schlitteninduktorium erzeugte faradische Strom. Um sichere Vergleichswerte vor und nach den Jodthyreoglobulininjektionen zu erhalten, wurde stets gleich lang gereitzt, und zwar meist 10 Sekunden, welche an einem Chronographen abgelesen wurden. Es soll auch ausdrücklich betont werden, dass bei allen 
Versuchen stets Sorge getragen wurde, dass die Elektroden (Platinhandelektroden) vor und nach der Zufuhr der zu prüfenden Substanz an der gleichen Stelle des Nerven und in gleicher Weise angelegt wurden. Der intakt belassene oder durchschnittene Nerv wurde an der Schlinge oder am abgebundenen Stumpf möglichst schonend zwischen den Muskeln emporgehoben und behufs Behinderung der Eintrocknung nach erfolgter Reizung sogleich in den Wundspalt zurückgebettet. Es wurde nach möglichst kurzer Vorprüfung eine Stromstärke gewählt, die einen eben wahrnehmbaren Effekt auslöste. Erwies sich dann die zu prüfende Substanz wirksam im Sinne einer Steigerung der Ansprechbarkeit des Nerven, so musste nach der Zufuhr der Ausschlag grösser sein. Dieser Modus hat sich am besten bewährt und ist auch der schonendste. Nicht geeignet, sichere Resultate zu geben ist der Modus, welcher darin besteht, dass man mit einer gegebenen Stromstärke einen bestimmten Effekt auslöst und nachher prüft, um wieviel schwächer der Strom gewählt werden muss, um den gleichen Effekt zu erzielen.

\section{Wirkung auf den Blutdruck.}

Zunächst sollte die Frage entschieden werden, ob Jodthyreoglobulin einen Einfluss auf den Blutdruck ausübe. Gelegenheit zum Studium eines Effektes nach dieser Richtung gab jeder der in dieser Abhandlung beschriebene Versuch.

Intravenöse Injektion von $0,2-0,5 \quad g$ Jodthyrenglobulin in $10-30 \mathrm{ccm}$ Wasser rief bei Tieren, die mit auf den Blutdruck wirkenden Substanzen nicht 'vorbehandelt worden waren, weder beim Kaninchen noch beim Hunde oder der Katze eine Blutdruckveränderung hervor ${ }^{1}$ ). Als Beispiele sollen folgende fünf Protokollauszüge hier angeführt werden.

Das Verhalten von Tieren, die mit auf den Blutdruck wirkenden Substanzen vorbehandelt worden waren; soll später zur Sprache kommen.

1) Dieser Befund stimmt nicht in allen Teilen zu den Angaben, die in einer früheren von E. v. Cy o $n$ und $m$ ir veröffentlichten Abhandlung (Über die physiologischen Wirkungen einiger aus der Schilddrüse gewonnener Produkte. Dieses Arch. Bd. 89 S. 199. 1901) niedergelegt sind. Doch trage ich für jene Publikation die Verantwortung nur für den chemischen Teil. 
Tabelle 1.

Versuch II. 19. Juli 1912. Kaninchen, Gewicht 2280 g. $8^{1 / 2}$ Uhr 2 g Urethan subkutan. Vagi und Depressores intakt.

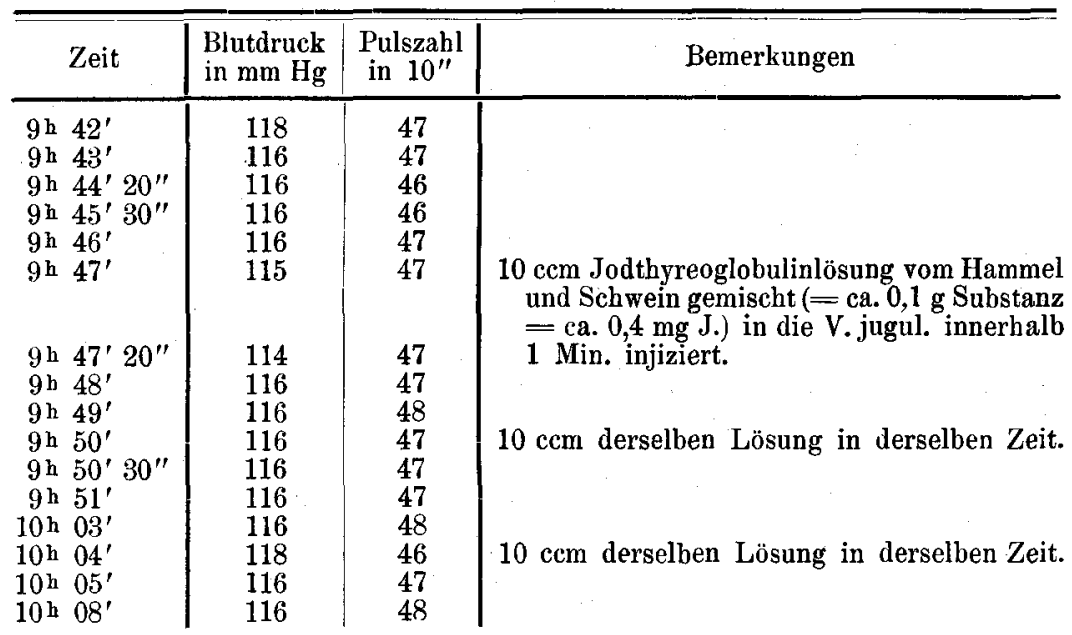

Tabelle 2 .

Versuch XVII. 4. Juli 1913. Kaninchen, Gewicht 2500 g. $9^{3 / 4}$ Uhr $2 \mathrm{~g}$ Urethan subkutan. Vagi und Depressores intakt.

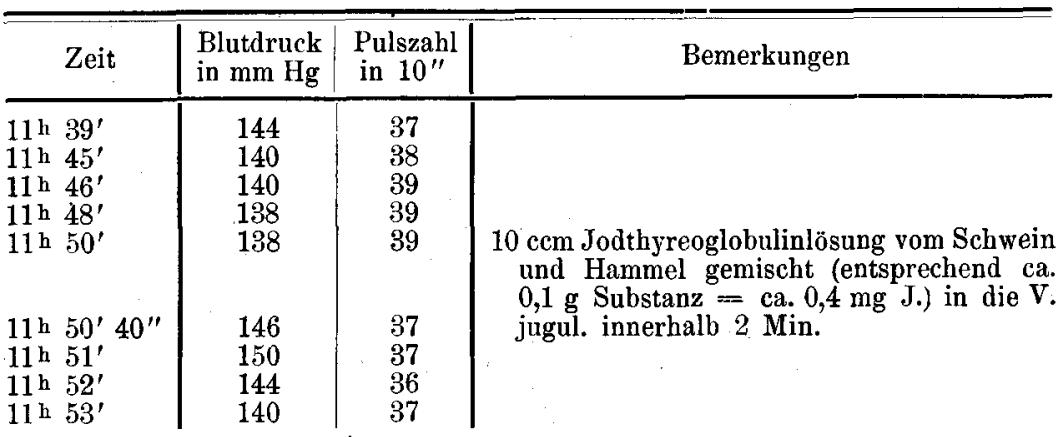

Tabelle 3 .

Vessuch XVIII. 5. Juli 1913. Kaninchen, Gewicht 2150 g. 101/4 Uhr $2 \mathrm{~g}$ Urethan subkutan. Vagi und Depressores intakt.

\begin{tabular}{|c|c|c|c|}
\hline Zeit & $\begin{array}{l}\text { Blutdruck } \\
\text { in } \mathrm{mm} \mathrm{Hg}\end{array}$ & $\begin{array}{l}\text { Pulszahl } \\
\text { in } 10^{\prime \prime}\end{array}$ & Bemerkungen \\
\hline $\begin{array}{l}12 \mathrm{~h} 02^{\prime} \\
12 \mathrm{~h} 05^{\prime}\end{array}$ & $\begin{array}{l}104 \\
104\end{array}$ & $\begin{array}{l}34 \\
35\end{array}$ & $\begin{array}{l}8 \mathrm{ccm} \text { Jodthyreoglobulinlösung vom Hammel } \\
\text { und Schwein gemischt (entsprechend ca. } \\
0,1 \mathrm{~g} \text { Substanz }=0,4 \mathrm{mg} \mathrm{J.)} \mathrm{in} \mathrm{die} \mathrm{Ohr-}\end{array}$ \\
\hline $\begin{array}{l}12 \mathrm{~h} 05^{\prime}, 20^{\prime \prime} \\
12 \mathrm{~h} 05^{\prime} 40^{\prime \prime} \\
12 \mathrm{~h} 06^{\prime} \\
12 \mathrm{~h} 06^{\prime}, 20^{\prime \prime} \\
12 \mathrm{~h} 06^{\prime}, 40^{\prime \prime} \\
12 \mathrm{~h} 07^{\prime}\end{array}$ & $\begin{array}{l}106 \\
108 \\
106 \\
100 \\
108 \\
108\end{array}$ & $\begin{array}{l}35 \\
34 \\
33 \\
34 \\
34 \\
34\end{array}$ & vene innerhalb 1 Min. \\
\hline
\end{tabular}




\section{Tabelle 4.}

Versuch XLII. 10. März 1914. Katze, Gewicht 2900 g. 31/2 Uhr $4 \mathrm{~g}$ Paraldehyd. Während der Operation Äthernarkose. Vagi und Depressores intakt.

\begin{tabular}{l|c|c|c}
\hline \hline Zeit & $\begin{array}{c}\text { Blutdruck } \\
\text { in mm Hg }\end{array}$ & $\begin{array}{c}\text { Pulszahl } \\
\text { in 10" }\end{array}$ & Bemerkungen \\
\hline 5 b $26^{\prime}$ & 83 & 30 & $\begin{array}{c}\text { 10 ccm Hammelsjodthyreoglobulin (entspre- } \\
\text { chend ca. 0,1 g Substanz =0,4 mg J.) } \\
\text { intravenös in 36". }\end{array}$ \\
5 h $26^{\prime}, 30^{\prime \prime}$ & 102 & 30 & \\
5 h $26^{\prime}, 40^{\prime \prime}$ & 104 & 30 & \\
5 h $27^{\prime}$ & 100 & 30 & \\
5 h 27' $30^{\prime \prime}$ & 99 & 30 & \\
5 h $28^{\prime}$ & 98 & 31 & \\
5 h $32^{\prime}$ & 96 & 30 &
\end{tabular}

Tabelle 5 .

Versuch XLIV. 13. Mai 1914. Hund, Gewicht $20 \mathrm{~kg}$. Paraldehyd. Vagi und Depressores intakt.

\begin{tabular}{|c|c|c|c|}
\hline Zeit & $\begin{array}{l}\text { Blutdruck } \\
\text { in } \mathrm{mm} \mathrm{Hg}\end{array}$ & $\begin{array}{l}\text { Pulszahl } \\
\text { in } 10^{\prime \prime}\end{array}$ & Bemerkungen \\
\hline $10^{\mathrm{h}} 51^{\prime}$ & 159 & 39 & $\begin{array}{l}10 \mathrm{ccm} \text { Jodthyreoglobulinlösung }(=0,2 \mathrm{~g} \\
\text { Substanz }=0,75 \mathrm{mg} \text { J.) intravenös in } 1^{?} \text {. }\end{array}$ \\
\hline $\begin{array}{l}10^{\mathrm{h}} 58^{\prime} \\
10^{\mathrm{h}} 59^{\prime}\end{array}$ & $\overline{170}$ & $\begin{array}{l}40 \\
40\end{array}$ & \\
\hline $10^{\text {h }} 59^{\prime} 30^{\prime \prime}$ & 180 & 41 & $\begin{array}{l}10 \mathrm{ccm} \text { derselben Jodthyreoglobulinlösung } \\
\text { in } 20^{\prime \prime} \text {. }\end{array}$ \\
\hline $11^{\text {h }} 00^{\prime} 30^{\prime \prime}$ & 187 & 40 & $\begin{array}{l}10 \mathrm{ccm} \text {,derselben Jodthyreoglobulinlösung } \\
\text { in } 35^{\prime \prime} \text {. }\end{array}$ \\
\hline $\begin{array}{l}11^{\text {h }} 01^{\prime}, 05^{\prime \prime} \\
11^{\text {h }} 08^{\prime}\end{array}$ & $\begin{array}{l}188 \\
198\end{array}$ & $\begin{array}{l}39 \\
41\end{array}$ & \\
\hline
\end{tabular}

Um die Angaben verschiedener Autoren [Oliver und Schäfer ${ }^{1}$ ) Haškovec ${ }^{2}$ ), Georgiewsky ${ }^{3}$ ), Fennyvessy ${ }^{4}$, Kraus und

1) E. A. Schäfer, On internal secretion. Brit. Med. Journ. vol. II p. 343. 1895. Siehe auch Oliver und $\mathrm{Sch}$ äfer, On the physiological action of extract of pituitary body and certain other glandular organs. Journ. of Physiol. vol. 18 p. 277. 1895 .

2) L. Haškovec, Über die Einwirkung des Schilddrüsensaftes auf den Kreislauf. Wiener med. Blätter Bd. 19 S. 111. 1896.

3) K. Georgiewsky, Über die Wirkung der Schilddrüsenpräparate auf den tierischen Organismus. Zeitschr. f. klin. Med. Bd. 33 S. 153. 1897.

4) B. v. Fennyvessy, Über die Wirkung des Schilddrüsensaftes anf die Zirkulation und Atmung nebst einem $\Lambda$ nhange über Beziehungen zwischen Jodothyrin und Jodnatrium, bzw. Atropin. Wiener klin. Wochenschr. 1900 S. 125. 
Friedenthal ${ }^{1}$ ) nachzuprufen, welche alle eine (geringe) Blutdrucksenkung (als konstante Erscheinung) nach intravenöser Injektion yon Schilddrüsen extrakten (aus frischen oder trockenen Drüsen) bei Hunden und Kaninchen angeben, habe ich wässerigen Extrakt aus frischen Hammel- und Hundeschilddrüsen Kaninchen intravenös injiziert. Die Extrakte wurden so hergestellt, dass gleiche Gewichtsteile Organbrei und physiologische Kochsalzlösung vermengt, 2 Stunden im Eisschrank gehalten wurden und danach die Lösung durch ein Tuch geseit und abgepresst wurde. 5 ccm solchermaassen dargestellte Extrakte führten bei den Versuchstieren keine oder nur eine unbedeutende Depression herbei. Wohl aber ergab sich eine solche (in geringem Umfang) bei der Verwendung enteiweisster Extrakte. Das ist wohl so zu erklären, dass die durch Kochen und Essigsäurezusatz eiweissfrei gemachten Filtrate vor ihrer Anwendung etwas eingeengt wurden, also konzentrierter waren. Wichtig dabei ist jedenfalls, dass eine depressorische Wirkung beobachtet werden konnte, dass diese aber dem eiweissfreien Extrakte innewohnte. Erwähnt soll werden, dass auch Oliver und Schäfer ihre Beobachtung am eiweissfreien Extrakte gemacht haben.

Da bekannt ist, dass auch andere (drüsige) Organe blutdruckerniedrigende Substanzen enthalten, wurden zum Vergleich Extrakte aus solchen (Nieren, Milz vom Hund) in gleicher Weise hergestellt und appliziert. Es zeigte sich dort der gleiche Effekt, und zwar kam auch dort eine Wirkung erst nach der Enteiweissung und dem schwachen Einengen deutlich zum Ausdruck. Sie war für die Nieren und Milz etwas stärker als für die Schilddrüse. Folgende Tabelle gibt die ziffernmässige Darstellung der gewonnenen Resultate wieder.

Tabelle 6.

Versuch LII. 11. Dézember 1914. Kaninchen, Gewicht 3050 g.

\begin{tabular}{|c|c|c|c|}
\hline Nr: & Zeit & $\begin{array}{l}\text { Blutdruck } \\
\text { in mm Hg }\end{array}$ & Bemerkungen \\
\hline 1 & $\begin{array}{l}4^{\text {h }} 55^{\prime} \\
4 \text { h } 55^{\prime} 10^{\prime \prime} \\
4^{\text {h }} 55^{\prime}, 20^{\prime \prime} \\
4^{\text {h }} 55^{\prime}, 30^{\prime \prime} \\
4^{h} 55^{\prime} 40^{\prime \prime}\end{array}$ & $\begin{array}{l}82 \\
82 \\
82 \\
79 \\
80\end{array}$ & $\begin{array}{l}5 \text { ccm wässeriger Extrakt aus frischen Hammels- } \\
\text { schild drüsen intravenös in } 32^{\prime \prime} \text {. }\end{array}$ \\
\hline
\end{tabular}

1) F. Kraus und Friedenthal, Über die Wirkung der Schilddrüsenstoffe. Berliner klin. Wochenschr. 1908 Nr. 38. 


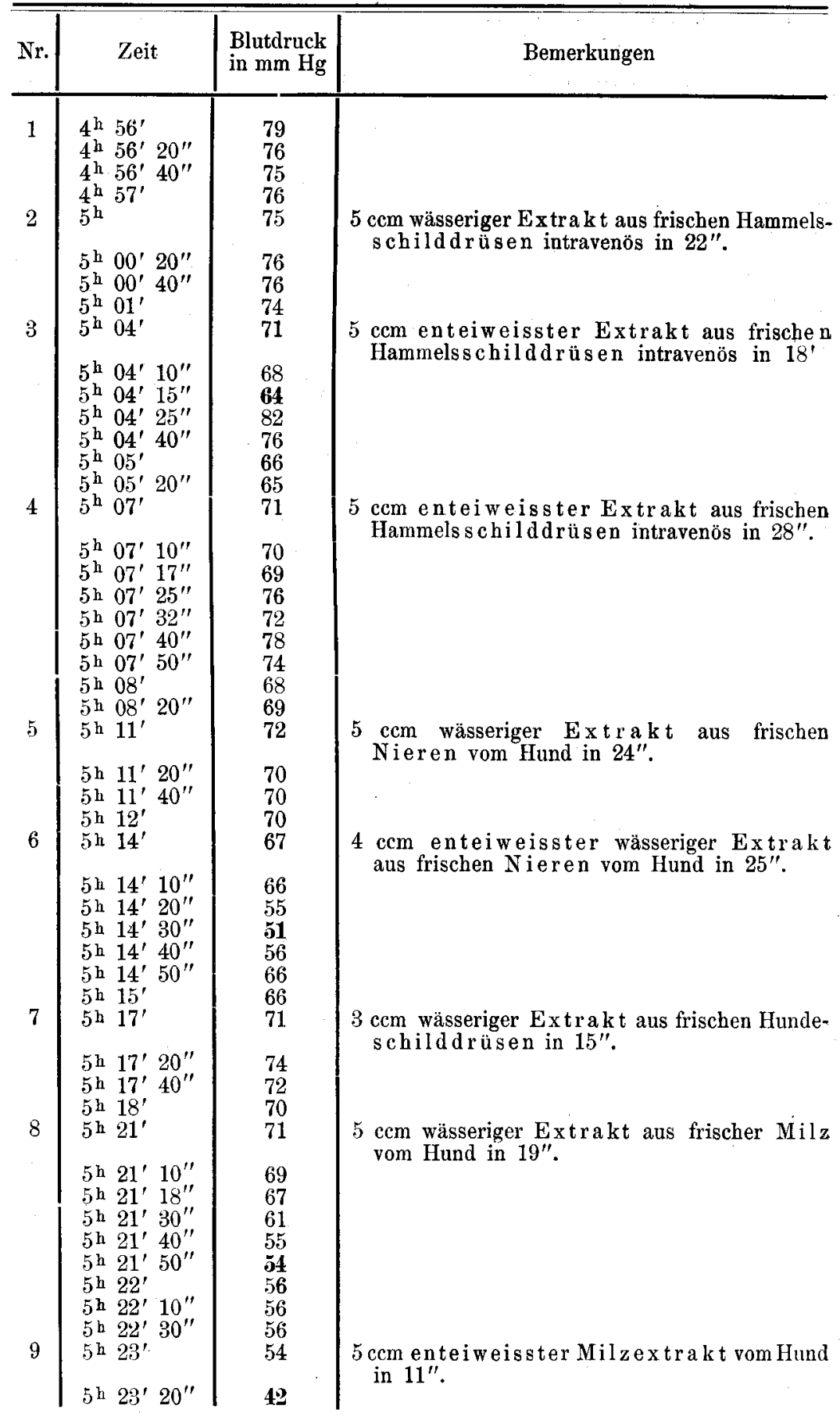




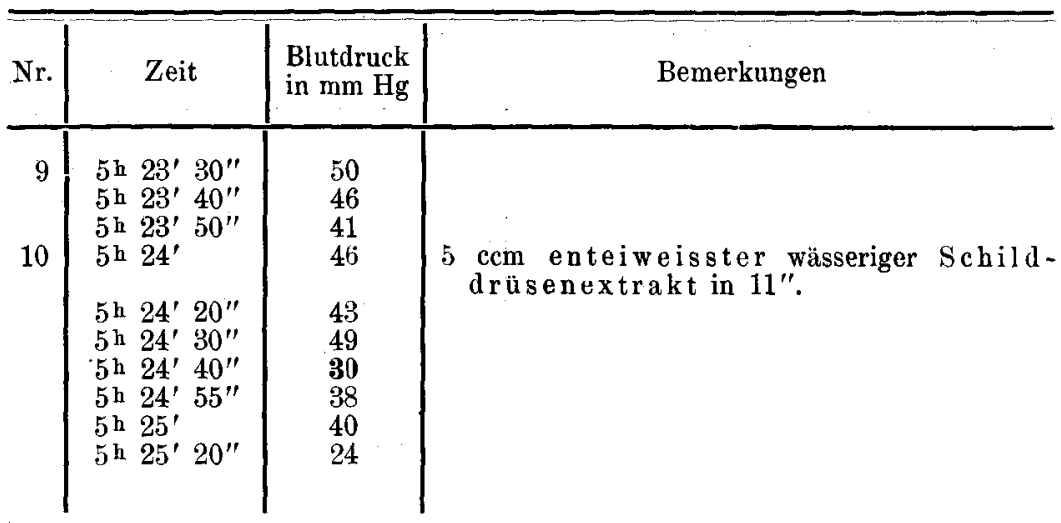

Fig. 1 gibt die Wirkung des enteiweissten Schilddrüsenextraktes beim Kaninehen wieder. In Fig. 2 ist zum Vergleich die Wirkung des enteiweissten Nierenextraktes abgebildet.

Aus dem Mitgeteilten ersehen wir, dass die depressorische Wirkung des Schilddrüsenextraktes nichts Charakte. ristiscbes für dieses Organ darstellt und dass sie nicht dem Jodthyreoglobulin innewohnt.

In Übereinstimmung mit diesen Schlussfolgerungen haben v. Fürth und Schwarz ${ }^{1}$ ), Lohmann ${ }^{2}$ ) und Gautrelet ${ }^{3}$ ) im Extrakt der Schilddrüse wie auch anderer drüsiger Organe enthaltenes Cholin dafür verantwortlich gemacht. Mit welchem Recht dies geschieht, soll in einer anderen Mitteilung dargetan werden.

Entsprechend diesen Feststellungen habe ich auch das Ba umann' sche Jodothyrin als unwirksam auf den Blutdruck befunden. Die Beobachtungen wurden an Kaninchen angestellt. Folgende Tabellen geben zwei Versuchsprotokolle wieder.

1) v. Fürth und Schwarz, Über die Einwirkung des Jodothyrins auf den Zirkulationsapparat. Dieses Arch. Bd. 124 S. 113. 1908.

2) A. L o h mann, Zur Physiwlogie der Schilddrüse, Sitzungsber. d. Gesellsch. z. Förderung d. ges. Naturw. in Marburg. 25. Mai 1908.

3) J. Gautrelet, Présence de la choline dans certaines glandes. Compt. rend. Soc. Biol. 1908 p. 174. - Mécanisme de l'action hypotensive de certaines glandes. Ibid. p. 174. 

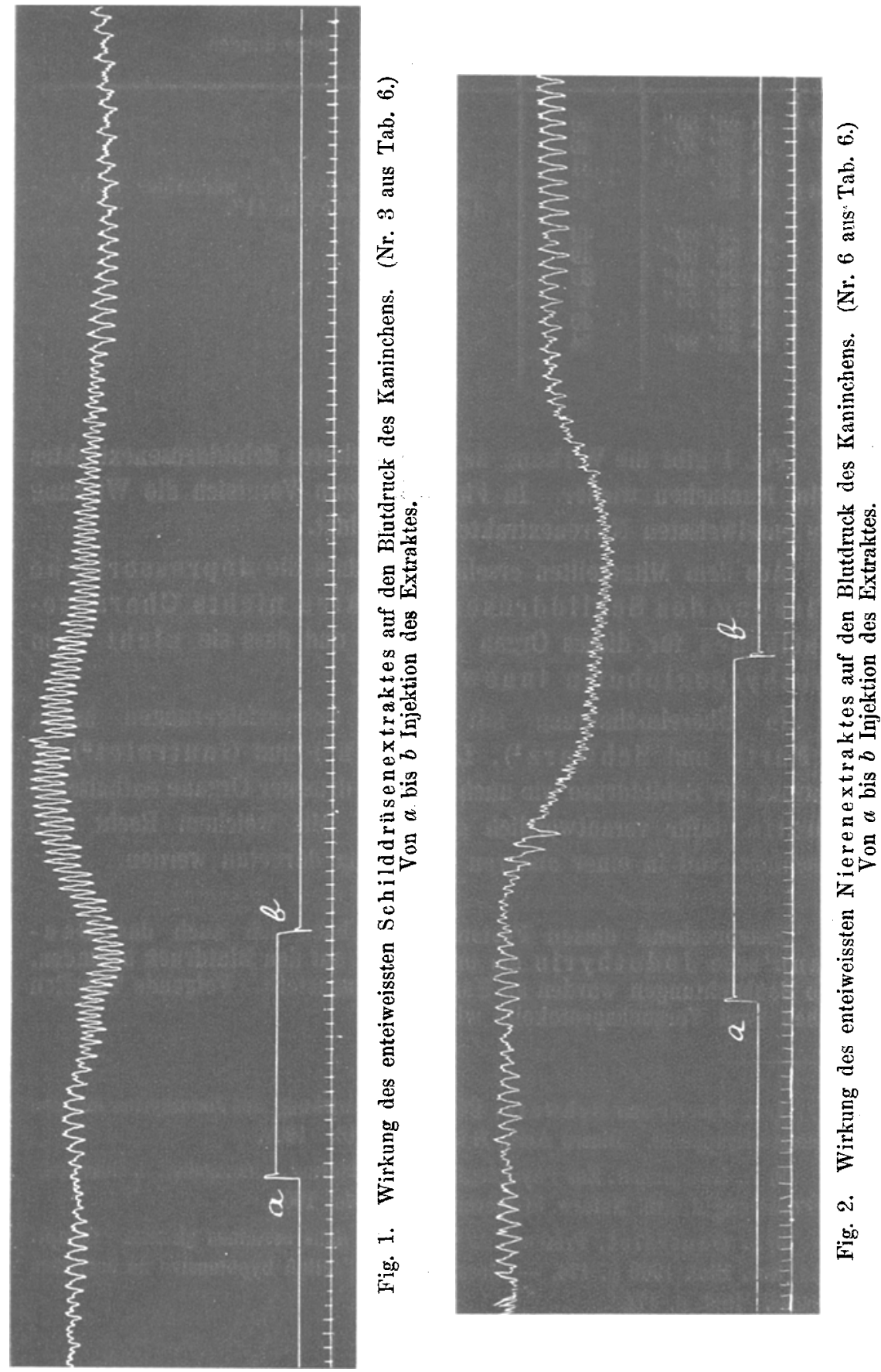
Tabelle 7.

Versuch VII. 27. Mai 1913. Kaninchen, Gewicht 2250 g. 3 Ubr 1,5 g Urethan sublutan.

\begin{tabular}{|c|c|c|c|c|}
\hline $\mathrm{Nr}$ & Zeit & $\begin{array}{l}\text { Blutdiruck } \\
\text { in } \mathrm{mm} \mathrm{Hg}\end{array}$ & $\begin{array}{l}\text { Pulszahl } \\
\text { in } 10^{\prime \prime}\end{array}$ & Bemerkungen \\
\hline 1 & 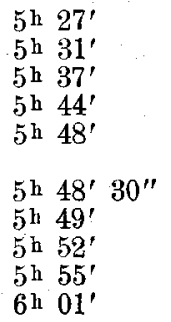 & $\begin{array}{l}64 \\
65 \\
64 \\
64 \\
65 \\
\\
70 \\
70 \\
68 \\
64 \\
66\end{array}$ & $\begin{array}{l}37 \\
37 \\
36 \\
35 \\
36 \\
\\
36 \\
36 \\
35 \\
35 \\
36\end{array}$ & $\begin{array}{l}6 \text { ccm Jod o th y rin lösung (=-1,8 mg } \\
\text { J.) intravenös in } 1^{\prime} \text {. }\end{array}$ \\
\hline
\end{tabular}

Tabelle 8 .

Versuch X. 5. Juni 1913. Kaninchen, Gewicht 2780 g. 3 Uhr 2 g Urethan subkutan.

\begin{tabular}{|c|c|c|c|c|}
\hline $\mathrm{Nr}$ & Zeit & $\begin{array}{l}\text { Blutdruck } \\
\text { in } \mathrm{mm} \mathrm{Hg}\end{array}$ & $\begin{array}{l}\text { Pulszahl } \\
\text { in } 10^{\prime \prime}\end{array}$ & Bemerkungen \\
\hline 1 . & $\begin{array}{l}\text { 6h } 29^{\prime}, 30^{\prime \prime} \\
6 \text { h } 30^{\prime} \\
\text { 6h } 30^{\prime} 30^{\prime \prime} \\
6 \text { h } 31^{\prime} \\
\text { 6h } 31^{\prime}, 30^{\prime \prime} \\
6 \text { h } 32^{\prime} \\
6 \text { h } 33^{\prime}\end{array}$ & $\begin{array}{r}100 \\
99 \\
\\
100 \\
108 \\
103 \\
104 \\
103\end{array}$ & $\begin{array}{l}37 \\
39 \\
\\
39 \\
38 \\
39 \\
38 \\
39\end{array}$ & $\begin{array}{l}5 \mathrm{ccm} \text { Jo d o thy rin lösung }(=1,5 \mathrm{mg} \\
\text { J.) intravenös in } 2^{\prime} \text {. }\end{array}$ \\
\hline
\end{tabular}

Diese Beobachtungen stimmen mit denen Kobert's ${ }^{1}$ ), v. Vamossy's und Vas's), Fennyvessy's ${ }^{3}$ ), v. Fürth's und Schwarz's ${ }^{4}$ ) überein, die alle eine Wirkung des Jodothyrins auf den Blutdruck in Abrede stellten. Nur v. Cyon ${ }^{5}$ ) beobachtete Blutdrucksenkung. Er verwendete $\mathrm{zu}$ seinen Versuchen eine Auflösung von Jodothyrin in Natriumkarbonatlösung. Fennyvessy konnte bei Wiederholung seiner Versuche jedoch den Befund nicht bestätigen. Wohl konnte er feststellen, dass bei Verwendung sehr grosser Mengen der gebrauchten (natriumkarbonathaltigen) Jodothyrinlösung eine Druck-

1) Siehe Schuster, Diskussion zu dem Referat: Über die therapeutische Anwendung der Schilddrüsenpräparate. Verhandl. d. Kongr. f. inn. Med. 1896 S. 153.

2) v. Vamossy und Vas, Experimentelle Untersuchungen über die Wirkung des Jodothyrins. Münchener med. Wochenschr. $1897 \mathrm{Nr} .25$.

3) $1 . \mathrm{c}$.

4) $1 . \mathrm{c}$.

5) v. Cyou, Beitrag zur Physiologie der Schilddrüse und des Herzens. Dieses Arch. Bd. 70 S. 165. 
senkung sich einstellte, die aber auch eintrat, wenn an Stelle der genannten Lösung die gleiche Menge einer entsprechend starken, wässerigen Lösung von Natriumkarbonat verwendet wurde. Somit handelte es sich nicht um eine Jodothyrinwirkung.

In die oben angeführte Aufzählung der Autoren, welche nach Schilddrüsenextrakt eine Blutdruckemiedrigung beobachtet haben, ist die Untersuchung Guinard's und Martin's (Actions cardio-vasculaires du suc thyrö̈dien. Compt. rend. de la soc. de biol. 1899 p. 161, siehe auch Contribution à l'étude des effets du suc surrénal. Journ. de Physiol. et Pathol. génér. t. 1 p. 748. 1899) nicht aufgenommen worden. Die beiden Autoren beobachteten nach der Injektion von $2 \mathrm{ccm}$ wässerigem Extrakt aus einer einem frisch hingerichteten Menschen entnommenen Schilddräse beim Hund plötzliche Blutdrucksenkung von $173 \mathrm{~mm} \mathrm{Hg}$ auf $110 \mathrm{~mm}$ unter Beschleunigung (von 138 auf 312) und Kleinwerden des Pulses. Ein so starker und plötzlicher Sturz ist nicht das Gewöhnliche. Dieses Verhalten erinnert an die Wirkung von Thymusextrakt, doch fällt auf, dass mehrere nachfolgende Injektionen von $4-14 \mathrm{ccm}$ des gleichen Extraktes keine erneute Depression erzeugten. Ein gleiches Verhalten wurde sonst von keinem anderen Experimentator beobachtet.

Gegenüber den angeführten, auf eine Blutdruckerniedrigung hinweisenden Befunden liegen Beobachtungen vor, welche eine Blutrirucks teig e r ung durch Schilddrüsenextrakt melden. [H e in a t $\mathrm{z}^{1}$ ), Livon ${ }^{2}$ ), Patta ${ }^{3}$ ).] Was die Resultate Heinatz' anbelangt, so sollen sie später besprochen werden, sie werden erst durch Verhältnisse geklärt, welche sich aus den nachfolgenden Schilderungen ergeben.

Die Befunde Livon's sind nur sehr summarisch mitgeteilt. Aus der kurzen Darlegung lässt sich nichts über die Methodik und den Verlauf der Experimente entnehmen. Livon teilt nur mit, dass er "hypertension et ralentissement (du pouls) très marqués" beobachtet habe und erwäbnt als Beispiel einen Fall, einen Hund betreffend, wo der Blutdruck von $200 \mathrm{~mm} \mathrm{Hg}$ auf $290 \mathrm{~mm}$ gestiegen sei (in wieviel Zeit wird nicht angegeben). Ein gleicher Befund (Drucksteigerung verbunden mit Pulsverlangsamung) ist von keinem anderen Experimentator erhoben worden. Auffallend ist, dass Livon auch Extrakte von Milz, Niere und, wie sich aus einer anderen Notiz ergibt ${ }^{4}$ ), von Parotis mit blutdrucksteigernden Eigenschaften begabt findet.

1) Hein a tz, Altes und Nenes über die Schilddrüse. Inaug.-Diss. (russisch). 1894.

2) Livon, Sécrétions internes; glandes hypertensives. Compt: rend. de la soc. de biol. 1898 p. 98 .

3) A. Patta, Contributo critico e sperimentale allo studio dell' azione degli estratti di organi sulla funzione circolatoria. Arch. di Farm. lib. 6 p. 102.1907.

4) Livon, Sécrétions internes; glandes hypotensives. Compt. rend. de la soc. de biol. 1898 p. 135. 
Die Abhandlung Patta's war mir im Original nicht zugänglich. Er fand, dass salzhaltige, wässerige Extrakte aus frischen Schilddrüsen oder aus Merck'schem Thyreoidin bei Hunden und Kaninchen bisweilen eine Drucksenkung, bisweilen eine Erhöhung oder aber erst eine Erhöhung und dann eine Senkung verursachen. Die Natur der Erhöhung kann ich ohne Kenntnis der Originalmitteilung nicht beurteilen.

\section{Wirkung auf den Herzrhythmus.}

Eine weitere Frage war die, ob Zufuhr von Jodthyreoglobulin eine Veränderung der Pulsgrösse und -zahl herbeiführt. Meine Versuche haben ergeben, dass die intravenöse Infusion von $10-30 \mathrm{ccm}$ Jodtbyreoglobulinlösung, enthaltend $0,2-0,5 \mathrm{~g}$ Substanz, bei Kaninchen, Hunden und Katzen in der Regel nach dieser Richtung wirkungslos waren. Tabelle $1-5$ geben aus den fünf schon angeführten Versuchen die Pulszahl vor und nach der Injektion wieder. Die Zahl ist nach wie vor die gleiche. Auch die Grösse der Pulswellen bleibt die gleiche. Letztere Ziffern wurden in die Tabellen nicht aufgenommen.

Genau das gleiche gilt auch für wässerige Vollextrakte aus der Schilddrüse. Folgende Tabelle gibt die Pulszahl vor und nach der Injektion von Schilddrüsenextrakt und zum Vergleich auch diejenige nach Injektion von Nieren- und Milzextrakten, alle vom Menschen.

$$
\text { Tabelle } 9 \text {. }
$$

Versuch LVI. 27. Oktober 1915. Kaninchen, Gewicht 2100 g. $91 / 2 \mathrm{Uhr}$ $1 \mathrm{~g}$ Urethan subkutan. Vagi und Depressores intakt.

\begin{tabular}{|c|c|c|c|}
\hline Nr. & Zeit & $\begin{array}{l}\text { Pulszahl } \\
\text { in } 10^{\prime \prime}\end{array}$ & Bemerkungen \\
\hline 1 & $\begin{array}{l}11^{\mathrm{h}} 03^{\prime} \\
11^{\mathrm{h}} 03^{\prime} \\
0^{\prime \prime}\end{array}$ & $\begin{array}{l}34 \\
33\end{array}$ & $\begin{array}{l}1 \mathrm{ccm} \text { wässeriger Schilddrüsenextrakt enteiweisst } \\
\text { in die Ohrvene. }\end{array}$ \\
\hline $\begin{array}{l}2 \\
3\end{array}$ & $\begin{array}{ll}11 \text { h } 04^{\prime}, 30^{\prime \prime} \\
11^{\mathrm{h}} 05^{\prime}\end{array}$ & $\begin{array}{l}32 \\
29\end{array}$ & $\begin{array}{l}3 \mathrm{ccm} \text { desselben Extraktes. } \\
4 \mathrm{ccm} \text { desselben Extraktes. }\end{array}$ \\
\hline 4 & $\begin{array}{l}11 \text { l } 00 \\
11 \text { h } 077^{\prime} \\
11 \text { h } 07^{\prime} 30^{\prime \prime}\end{array}$ & $\begin{array}{l}35 \\
32 \\
33\end{array}$ & $1 \mathrm{ccm}$ Nierenextrakt (enteiweisst). \\
\hline $\begin{array}{l}5 \\
6\end{array}$ & $\begin{array}{l}11 \mathrm{~h} 08^{\prime} \\
11 \mathrm{~h} 09^{\prime}, 30^{\prime \prime}\end{array}$ & $\begin{array}{l}32 \\
29 \\
31\end{array}$ & $\begin{array}{l}3 \mathrm{ccm} \text { desselben Extraktes. } \\
6 \mathrm{ccm} \text { desselben Extraktes. }\end{array}$ \\
\hline 7 & $\begin{array}{l}11 \text { h } 15^{\prime} \\
11 \text { h } 17^{\prime}\end{array}$ & 80 & $10 \mathrm{ccm}$ Milzextrakt (enteiweisst). \\
\hline
\end{tabular}


Wie sich daraus ergibt, ist die Pulszahl vor und nach der intravenösen Beibringung von Schilddrüsenextrakt bei Kaninchen die gleiche. Ausserdem soll bemerkt werden, dass die Injektion der Extrakte keinen Finfluss auf die Pulsgrösse hatte.

Im Gegensatz zu dem, Gesagten traten nach Jodthyreoglobulinzufuhr in seltenen Fällen (bei Kaninchen) vorübergehend grosse Pulse auf unter entsprechender Abnahme der Zahl. Folgender Protokollauszug veranschaulicht dieses Verhalten.

Tabelle 10.

Versuch III. 23. Juli 1912. Kaninchen, Gewicht $2550 \mathrm{~g}$. 8 Uhr $2 \mathrm{~g}$ Urethan subkutan. Vagi und Depressoren intakt.

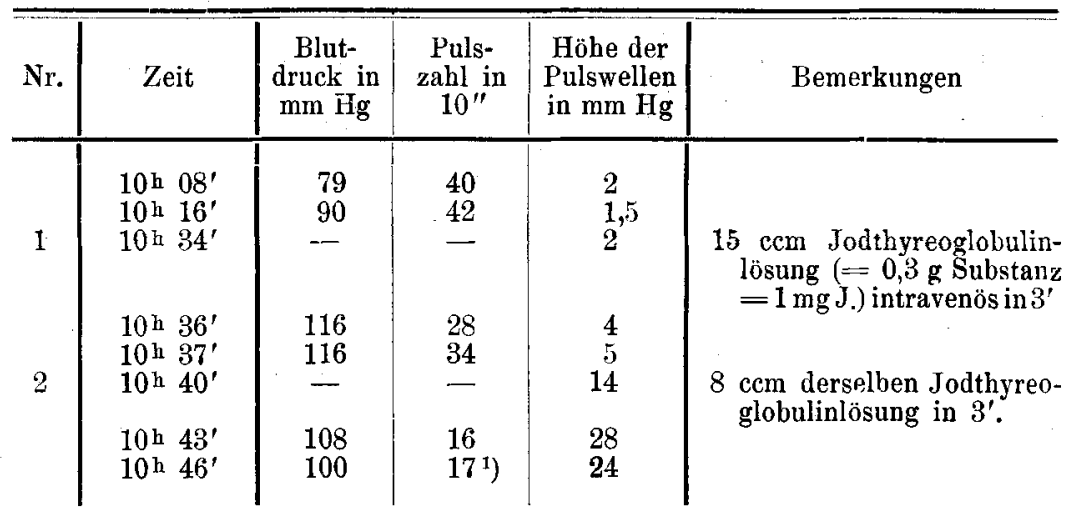

Zwei Kurvenausschnitte sind auf Fig. 3 und 4 abgebildet.

Das Auftreten solcher Pulse dürfte auf eine besondere Empfindlichkeit des Vagus hindeuten. Ein solcher individueller Unterschied gegenüber elektrischen und mechanischen Reizen ist dem Tierexperimentator schon lange bekannt und ist auch in den jüngsten Jahren am lebenden Menschen gegenüber Vagusmitteln festgestellt worden. Das abweichende Verbalten soll später noch zur Sprache kommen und wird aus dem später Mitgeteilten leichter verständlich sein.

1) Es soll bemerkt werden, dass der Jodthyreoglobulinzufuhr mehrmals eine Vagusreizung vorausgegangen war, deren manifeste Wirkung jedoch vor der Injektion ausgeklungen war. Ich will nicht behaupten, dass dieser Umstand am Anftreten der Vaguspulse schuld gewesen sei, sondern begnüge mich damit, die Tatsache anzuführen. Wir werden sehen, dass auch bei anderen Wirkungen die Antezedenzien durch das Jodthyreoglobulin zum Ausdruck gebracht werden. 
Auf dieses Verhalten ist wohl die bei mehreren früheren Autoren [Georgiewsky ${ }^{1}$ ), Livon ${ }^{2}$ ), Fennyvessy ${ }^{3}$ ), Ocaña ${ }^{4}$ ), Patta ${ }^{5}$ ), Haškovec $\left.{ }^{6}\right)$ ] zu findende Angabe einer Pulsverlangsamung nach intravenöser Zufuhr von Schilddrüsenextrakt zurückzuführen. Leider ist bei den meisten nichts über die Grösse der Pulswellen ausgesagt, wenngleich bei einigen der Hinweis auf eine Vaguswirkung zu finden ist.

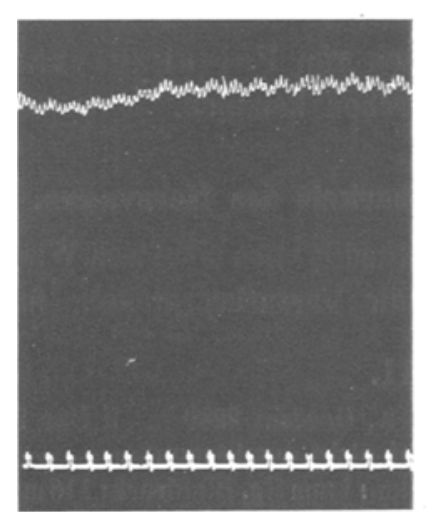

Fig. 3. Kaninchen. Pulskurve vor der Jodthyreoglobulininjektion.

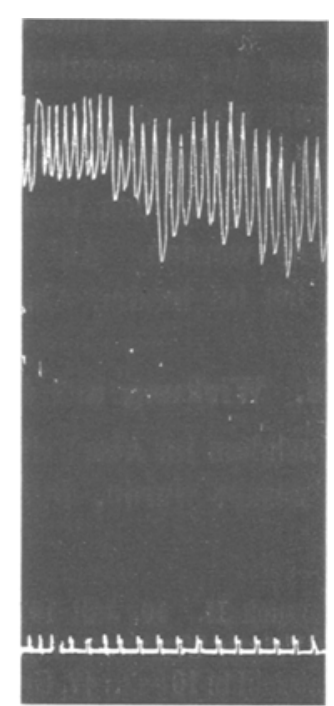

Fig. 4. Kaninchen. Pulskurve nach der Jodthyreoglobulininjektion.

Im Gegensatz $\mathrm{zu}$ diesen Angaben stehen andere [Heinat ${ }^{7}$ ), Haškovec ${ }^{8}$ ), Guinard und Martinn ${ }^{9}$ ), Svebla $\left.{ }^{10}\right)$ ], welche

1) l. c.

2) $1 . \mathrm{c}$.

3) l. c.

4) G. Ocaña, Sur les sécrétions internes des glandes avec et sans canal excréteur et même des organes non-glandulaires. Physiologen-Kongress, Turin 1902.

5) l. $\mathrm{c}$.

6) L. Hašovec, Über Wirkung des Thyreoidalextraktes. Wiener klin. Wochenschr. $1911 \mathrm{Nr} .31$ S. 1117.

7) l. c.

8) l. c.

9) l. c.

10) K. Svehla , Experimentelle Beiträge zur Kenntnis der inneren Sekretion der Thymus, der Schilddrüse und der Nebennieren von Embryonen und Kindern. Arch. f. exp. Pathol. u. Pharmak. Bd. 53 S. 321. 1900. 
eine Pulsbeschleunigung betreffen. Eine solche habe ich (bei Kaninchen) nie (in ca. 60 Versuchen) beobachtet, auch bei den wenigen Versuchen, die ich an Hunden und Katzen angestellt habe, nicht. Doch sind, was letztere beide Tierarten anbetrifft, meine Versuche zu wenig zahlreich, um abschliessend zu sein. Die bestimmten Angaben der Autoren sind durchaus glaubwürdig, sie stehen auch im Einklang mit dem, was wir über die Pathologie der Schilddrüse wissen. Es kommt hier jedenfalls auf die Tiergattung bzw. die Rasse an, namentlich dürfte es Hundearten geben, die leichter reagieren als andere, dafür haben wir schon bestimmte Anhaltspunkte ${ }^{1}$ ). Leider ist bisher zu wenig auf die Arten und Rassen geachtet und in den Protokollen der vorliegenden Arbeiten darüber vermerkt worden. Auf das Vorkommen von Pulsakzeleration werde ich im letzten Abschnitt zu sprechen kommen.

\section{Wirkung auf die Ansprechbarkeit des Herzvagus.}

Nachdem im Abschnitt über die Methodik alles technisch Wissenswerte gesagt wurde, lasse ich gleich die Versuchsprotokolle folgen.

Tabelle 11.

Versuch II. 19. Juli 1912. Kaninchen, Gewicht 2280 g. 9 Uhr $2 \mathrm{ccm}$ einer 20\% igen Urethanlösung subkutan. Vagi nicht durchschnitten.

Pulszahl in 10 Sek.: 47, Grösse der Pulswellen: $1 \mathrm{~mm} \mathrm{Hg}$, Blutdruck: $116 \mathrm{~mm} \mathrm{Hg}$.

\begin{tabular}{|c|c|c|c|c|c|c|c|}
\hline $\mathrm{Nr}$. & Zeit & 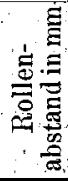 & 苞 & 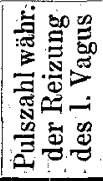 & 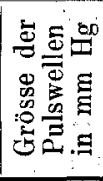 & 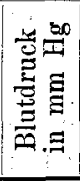 & Bemerkungen \\
\hline 1 & 9 h $44^{\prime}$ & 200 & $10^{\prime \prime}$ & 21 & 32 & - & \multirow{10}{*}{$\begin{array}{l}10 \text { ccm Jodthyreoglobulin- } \\
\text { lösung }(=0,2 \mathrm{~g} \text { Substanz, } \\
\text { entspr. } 0,7 \mathrm{mg} \text { J.) in } 1 \text {. } \\
10 \text { ccm der gleichen Jod- } \\
\text { thyreoglobulinlösung in } 1^{\prime} \text {. }\end{array}$} \\
\hline & 9 h $44^{\prime} 30^{\prime \prime}$ & & - & & 1 & 116 & \\
\hline 2 & $9 \mathrm{~h} 45^{\prime}$ & 200 & $9^{\prime \prime}$ & 15 & 13 & - & \\
\hline 3 & $\begin{array}{l}9 \text { h } 45^{\prime} 30^{\prime \prime} \\
9 \text { h } 46^{\prime}\end{array}$ & $\overline{200}$ & $\bar{g}^{\prime \prime}$ & $\overline{13}$ & $\begin{array}{r}1 \\
22\end{array}$ & 116 & \\
\hline & 9 h $47^{\prime}$ & & $\div$ & & 1 & - & \\
\hline & $9^{\mathrm{h}} 50^{\prime}$ & - & - & - & 1 & 一 & \\
\hline & 9 h $51^{\prime}$ & & $\overrightarrow{10 \prime \prime}$ & $\overline{11}$ & 1 & 116 & \\
\hline 4 & $\begin{array}{l}9 \text { h } 54^{\prime} \\
9 \text { h } 55^{\prime}\end{array}$ & 200 & $10^{\prime \prime}$ & 11 & ${ }_{44-48}^{54}$ & 一 & \\
\hline $\begin{array}{l}5 \\
6\end{array}$ & $\begin{array}{l}9 \mathrm{~h} 55^{\prime} \\
10 \mathrm{~h}\end{array}$ & $\begin{array}{l}200 \\
200\end{array}$ & $\begin{array}{l}10^{\prime \prime \prime} \\
7 "\end{array}$ & $\begin{array}{r}10 \\
8\end{array}$ & $\begin{array}{c}44-48 \\
42\end{array}$ & - & \\
\hline 7 & $10^{\mathrm{h}} 03^{\prime}$ & 200 & $10^{\prime \prime}$ & 10 & $46-50$ & 一 & \\
\hline
\end{tabular}

1) Guinard und Martin haben bei manchen Hunden Pulsakzeleration, bei anderen Retardation gefunden. Wahrscheinlich war das bei verschiedenen Rassen. 
Wie wir sehen, fällt die Pulszahl, die bei einem Rollenabstand von $200 \mathrm{~mm}$ bei 10 Sekunden langer Reizung in 10 Sekunden 21 betrug, nach der Injektion von $0,2 \mathrm{~g}$ Jodthyreoglobulin auf 10 und die Pulsgrösse wächst von $32 \mathrm{~mm} \mathrm{Hg}$ auf $50 \mathrm{~mm} \mathrm{Hg}$. Die Wirkung war nach 16 Minuten noch nicht erloschen.

Fig. 5 und 6 geben zwei Ausschnitte aus der Kurve wieder, den einen vor, den anderen nach der Jodthyreoglobulinzufuhr.

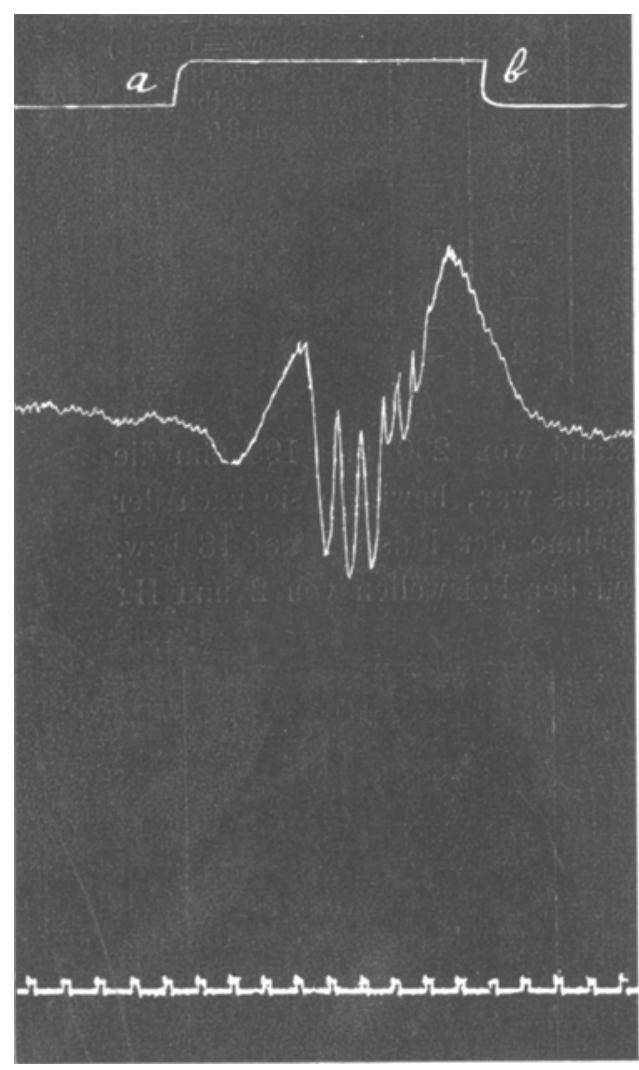

Fig. 5. Vagusreizung vor der Jodthyreoglobulinzufuhr. Nr. 1 aus Tab. 11. Von $a-b$ Dauer der Vagusreizung.

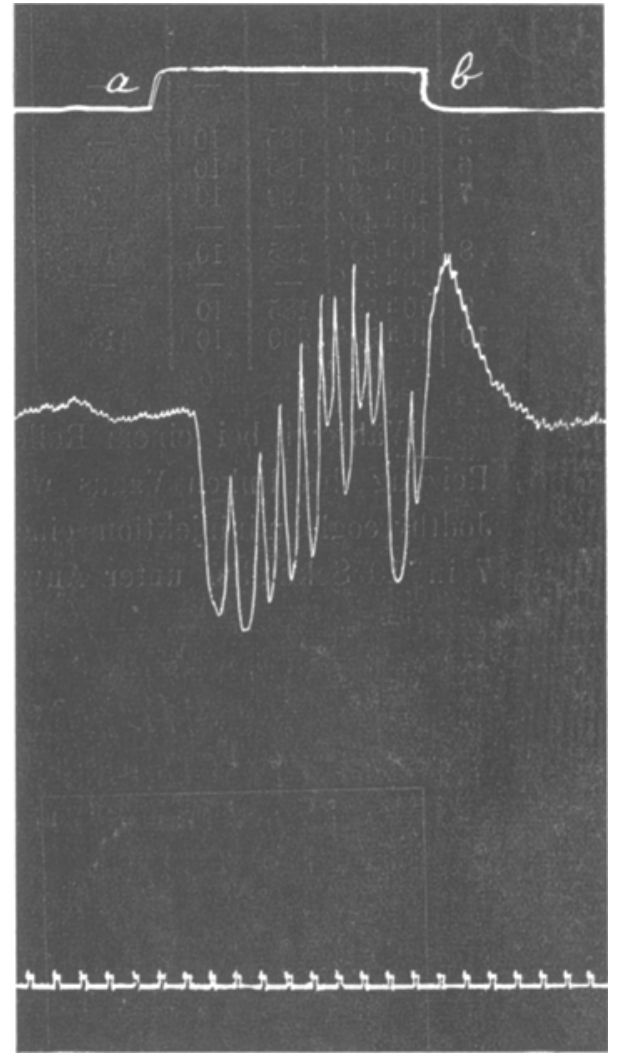

Fig. 6. Vagusreizung nach der Jodthyreoglobulinzufuhr. Nr. 5 aus Tab. 11 . Von $a-b$ Dauer der Vagusreizung.

\section{Tabelle 12.}

Versuch III. 23. Juli 1912. Kaninchen, Gewicht $2250 \mathrm{~g}$. 81/2 Uhr $2 \mathrm{~g}$ Urethan subkutan. 9 Uhr 50 Min. Beginn des Versuches. Vagi nicht durchschnitten. Pulszahl in 10 Sek.: 40, Grösse der Pulswellen: $2 \mathrm{~mm} \mathrm{Hg}$, Blutdruck: $84 \mathrm{~mm} \mathrm{Hg}$. 


\begin{tabular}{|c|c|c|c|c|c|c|c|c|}
\hline \multirow{2}{*}{$\mathrm{Nr}$. } & \multirow{2}{*}{ Zeit } & \multirow{2}{*}{ 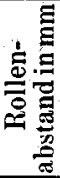 } & \multirow{2}{*}{ 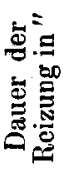 } & \multicolumn{2}{|c|}{$\begin{array}{l}\text { Pulszahl während } \\
\text { der Reizdauer }\end{array}$} & \multirow{2}{*}{ 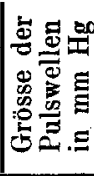 } & \multirow{2}{*}{ 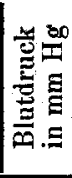 } & \multirow{2}{*}{ Bemerkungen } \\
\hline & & & & $\begin{array}{c}\text { d. linken } \\
\text { Vagus }\end{array}$ & $\begin{array}{c}\text { d.rechten } \\
\text { Vagus }\end{array}$ & & & \\
\hline 1 & $10 \mathrm{~h} 03^{\prime}$ & 200 & 10 & Kaine Wirknng & - & 2 & & \\
\hline 2 & $10^{\mathrm{h} 04^{\prime}}$ & 200 & 10 & Keine Wirkning & - & 2 & 83 & \\
\hline 3 & $10^{\mathrm{h}} 05^{\prime}$ & 190 & 10 & Loichto Depress. & & 2 & 83 & \\
\hline 4 & $10^{\mathrm{h}} 07^{\prime}$ & 185 & 10 & - & Keinelirkang & 2 & 79 & \\
\hline & $10^{\mathrm{h}} 34^{\prime}$ & - & - & - & - & 2 & - & $\begin{array}{c}15 \text { ccm Jodthyreoglo- } \\
\text { bulinlösung }(=0,3 \mathrm{~g} \\
\text { Substanz }=1 \mathrm{mg} \mathrm{J} .) \\
\text { intravenös in } 3^{\prime} \text {. }\end{array}$ \\
\hline & $10^{\mathrm{h}} 40^{\prime}$ & - & - & - & - & 一 & - & 8 cem derselben Lö- \\
\hline 5 & $10^{\text {h }} 44^{\prime}$ & 185 & 10 & - & 13 & 42 & - & \\
\hline 6 & $10^{\text {h }} 47^{\prime}$ & 185 & 10 & - & 15 & 33 & - & \\
\hline 7 & $10 \mathrm{~h} 48^{\prime}$ & 190 & 10 & 7 & - & 39 & $\overline{78}$ & \\
\hline 8 & $10 \mathrm{~b} 50^{\prime}$ & 185 & 10 & 10 & - & 43 & 18 & \\
\hline & & & & 8 & . & & 89 & \\
\hline 9 & $10^{\mathrm{h}} 52^{\prime}$ & 185 & 10 & - & 17 & 23 & - & \\
\hline 10 & $10^{\mathrm{h}} 53^{\prime}$ & 200 & 10 & 18 & - & 24 & - & \\
\hline
\end{tabular}

Während bei einem Rollenabstand von 200 und $190 \mathrm{~mm}$ die Reizung des linken Vagus wirkungslos war, bewirkte sie nach der Jodthyreoglobulininjektion eine Abnahme der Pulszahl auf 18 bzw. 7 in 10 Sekunden unter Anwachsen der Pulswellen von $2 \mathrm{~mm} \mathrm{Hg}$

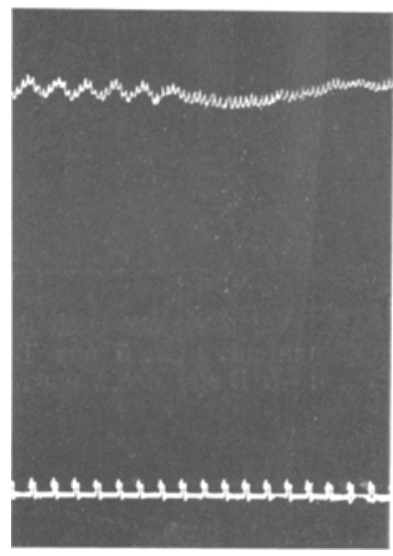

Fig. 7. Vagusreizung vor der Jodthyreoglobulininjektion. Nr. 3 aus Tab. 12.

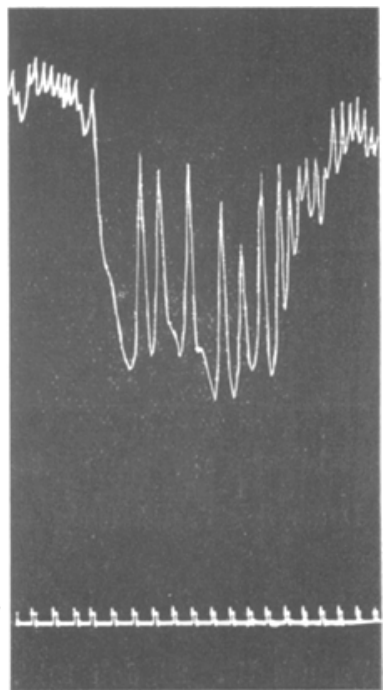

Fig. 8. Vagusreizung nach der Jodthyrenglobulininjektion. Nr. 8 aus Tab. 12. 
auf $24-39: \mathrm{mm} \mathrm{Hg}$. Rechterseits war die Reizung bei $185 \mathrm{~mm}$ Abstand erfolglos. Nach der Injektion fiel die Pulszahl auf 13 und 15 unter Zunahme der Pulsgrösse von $2 \mathrm{~mm} \mathrm{Hg}$ auf $33-42 \mathrm{~mm} \mathrm{Hg}$.

Fig. 7 und 8 geben zwei Aussehnitte ans der Kurve wieder, Fig. 7 vor, Fig. 8 nach der Jodthyreoglobulininjektion.

\section{Tabelle 13.}

Versuch IV. 25. Juli 1912. Kaninchen, Gewicht $2350 \mathrm{~g}$. 8 Uhr $2 \mathrm{~g}$ Urethan subkutan. Beginn des Versuches $10 \mathrm{Uhr} 5 \mathrm{Min}$. Vagi nicht durchschnitten. Pulszahl in 10 Sek.: 42, Grösse der Pulswellen: 1,7 mm Hg, Blutdruck: $65 \mathrm{~mm} \mathrm{Hg}$

\begin{tabular}{|c|c|c|c|c|c|c|c|c|}
\hline \multirow[b]{2}{*}{$\mathrm{Nr}$. } & \multirow[b]{2}{*}{ Zeit } & \multirow{2}{*}{ 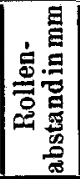 } & \multirow{2}{*}{ 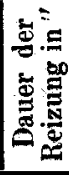 } & \multicolumn{2}{|c|}{$\begin{array}{c}\text { Pulszahl während } \\
\text { der Reizung }\end{array}$} & \multirow{2}{*}{ 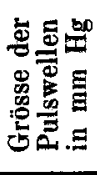 } & \multirow{2}{*}{ 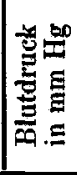 } & \multirow[b]{2}{*}{ Bemerkungen } \\
\hline & & & & $\begin{array}{l}\text { des } \\
\text { linken } \\
\text { Vagus }\end{array}$ & $\begin{array}{c}\text { des } \\
\text { rechten } \\
\text { Vagus }\end{array}$ & & & \\
\hline 1 & $10^{\mathrm{h}} 22^{\prime}$ & 200 & 10 & 26 & - & $3-15$ & - & \\
\hline 2 & $10^{\text {h } 22^{\prime} 30^{\prime \prime}}$ & 200 & 10 & - & 30 & $3-7$ & - & \\
\hline 3 & $10^{\mathrm{h}} 29^{\prime}$ & 200 & 10 & 16 & - & 18 & - & \\
\hline 4 & $10^{\mathrm{h}} 44^{\prime}$ & - & - & - & - & - & 84 & $\begin{array}{l}5 \text { ccm Jodthyreoglo- } \\
\text { bulinlösung }(=0,1 \mathrm{~g} \\
\text { Substanz }(=0,4 \mathrm{gJ} .) \\
\text { intravenös. }\end{array}$ \\
\hline 5 & $10 \mathrm{~h} 48^{\prime}$ & 200 & 10 & 2 & - & 22 & $\overline{05}$ & \\
\hline 6 & $\begin{array}{l}10^{\mathrm{h}} 48^{\prime} \\
10^{\mathrm{h}} 49^{\prime}\end{array}$ & 200 & 10 & - & 10 & 23 & 85 & \\
\hline & $10^{\text {h }} 49^{\prime} 30^{\prime \prime}$ & & & & & - & 82 & \\
\hline 7 & $10^{\mathrm{h}} 50^{\prime}$ & 200 & 10 & 1 & - & 31 & 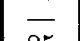 & \\
\hline 8 & $\begin{array}{l}10^{\mathrm{n}} 56 \\
10^{\mathrm{h}} 58^{\prime}\end{array}$ & 200 & 10 & - & $\overrightarrow{10}$ & 35 & 85 & \\
\hline
\end{tabular}

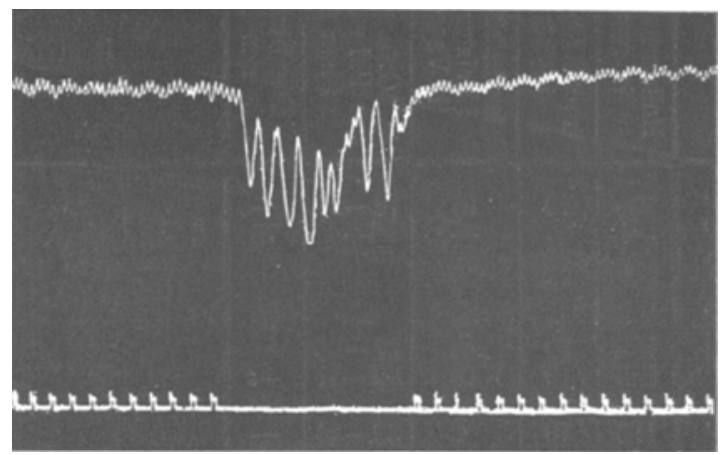

Fig. 9. Vagusreizung vor der Jodthyreoglobulinzufuhr. Nr. 3 aus Tab. 13.

Die Pulszahl sank bei gleichem Rollenabstand und gleicher Reizdauer in 10 Sekunden von 26 bzw. 16 bei Reizung des linken Vagus auf 2 und 1, bei Reizung dés rechten von 30 auf 10 . Zu- 
nahme der Pulsqrösse von $18 \mathrm{~mm} \mathrm{Hg}$ auf 31 , bzw. von 7 auf 35 . Nach 18 Minuten war die Wirkung nicht erloschen.

Fiy. 9 und 10 geben zwei Aussehnitte aus der Kurve.

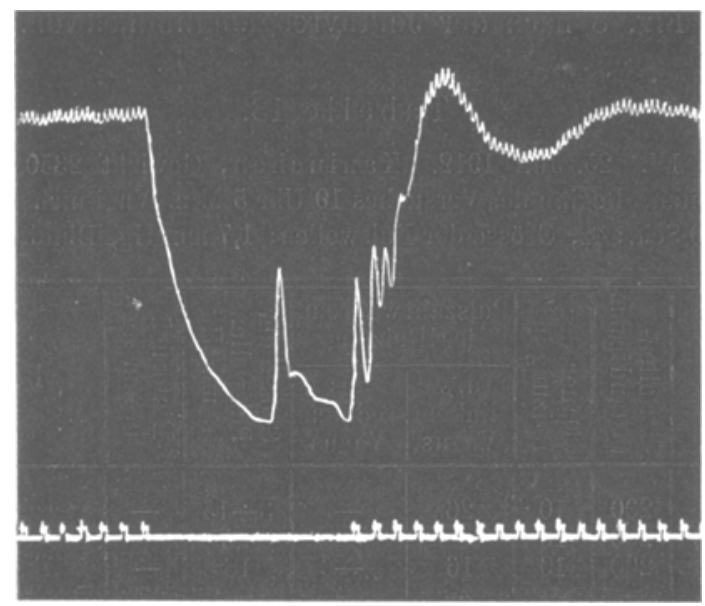

Fig. 10. Vagusreizung nach der Jodthyreoglobulinzufuhr. Nr. 7 aus Tab. 13.

$$
\text { Tabelle } 14 .
$$

Versuch XIII. 19. Juni 1913. Kan inchen, Gewicht 2750 g. 4 Uhr 2 g Urethan subkutan. Beginn des Versuches 5 Uhr 20 Min. Vagi intakt. Pulszahl in 10 Sek.: 44, Grösse der Pulswellen : $1 \mathrm{~mm} \mathrm{Hg}$.

\begin{tabular}{|c|c|c|c|c|c|c|c|}
\hline $\mathrm{Nr}$. & Zeit & 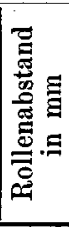 & 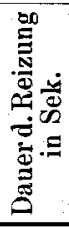 & 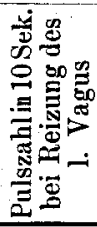 & 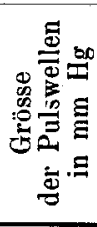 & 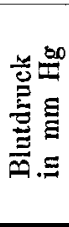 & Bemerkungen \\
\hline 1 & $\begin{array}{ll}5 \text { h } & 41^{\prime} \\
5 \text { h } & 49^{\prime}\end{array}$ & 185 & 10 & 16 & $\begin{array}{c}18-24 \\
-\end{array}$ & $\overline{76}$ & $\begin{array}{l}6 \mathrm{ccm} \text { Jodthyreoglobulinlösung } \\
(=0,15 \mathrm{~g} \text { Substanz } \\
0,5 \text { mg J.) intravenös in } 1^{\prime} .\end{array}$ \\
\hline 2 & $\begin{array}{ll}5 \text { h } & 50^{\prime} \\
5 \text { h } & 57^{\prime}\end{array}$ & $\overline{185}$ & $\overline{10}$ & $\overline{15}$ & $14-16$ & 76 & \\
\hline 3 & $\begin{array}{ll}6 \text { h } & 01^{\prime} \\
6^{\text {h }} & 06^{\prime}\end{array}$ & 185 & 10 & 12 & $\begin{array}{c}39-41 \\
-\end{array}$ & $\overline{90}$ & \\
\hline 4 & 6 h $07^{\prime}$ & 185 & 10 & 2 & $39-44$ & - & \\
\hline
\end{tabular}

Die Pulszahl sank von 16 auf 6 , die Pulsgrösse nahm zu von $18 \mathrm{~mm} \mathrm{Hg}$ auf $44 \mathrm{~mm}$. Die Wirkung war 18 Minuten nach der Injektion noch nicht erloschen.

Die Fig. 11 und 12 geben Ausschnitte aus der Kurve wieder. 


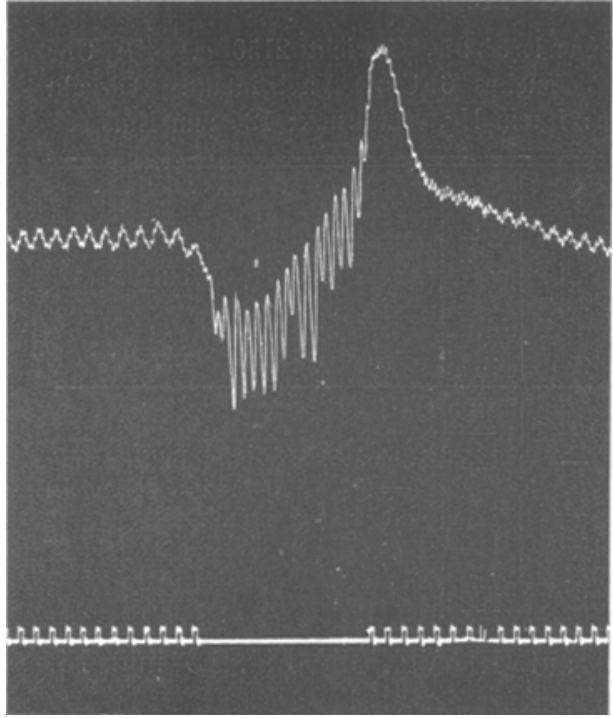

Fig. 11. Vaguswirkung vor der Jodthyreoglobulinzufuhr. Nr. 1 aus Tab. 14.

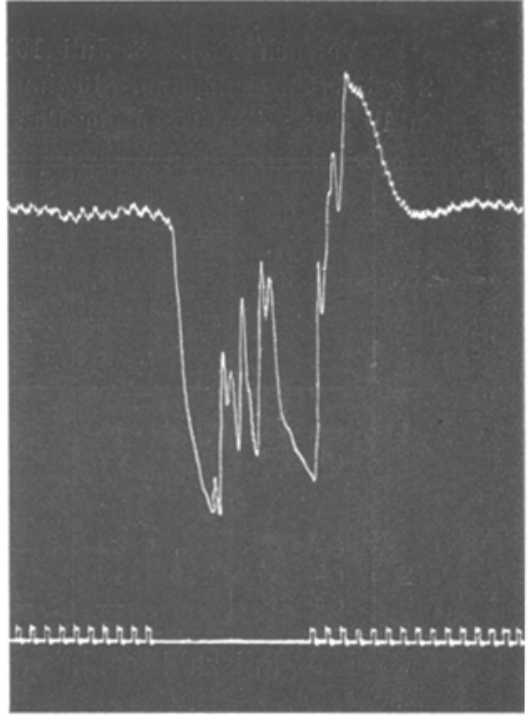

Fig. 12. Vaguswirkung nach der Jodthyreoglobulinzufuhr. Nr. 4 aus Tab. 14.

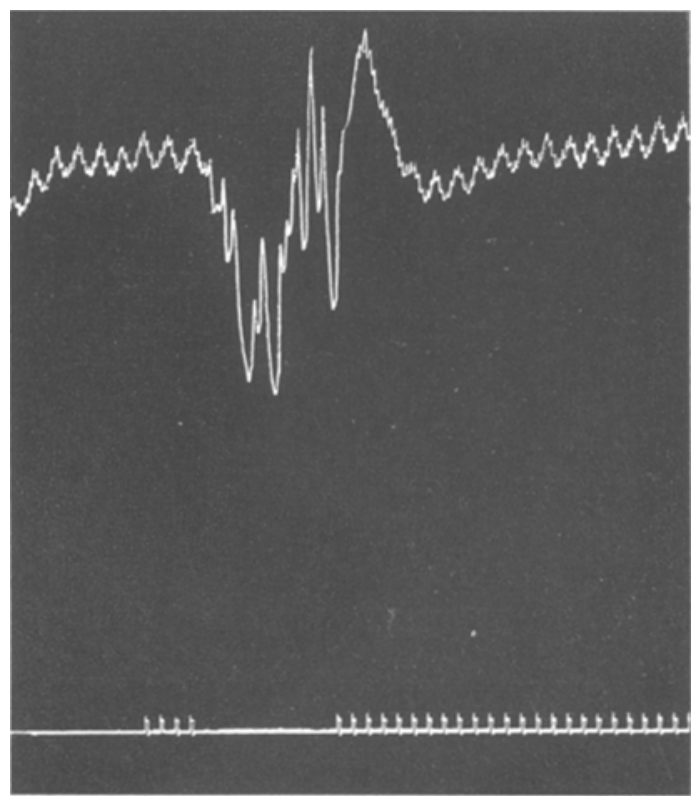

Fig. 19. Vaguswirkung vor der Jodthyreoglobulinzufuhr. Nr. 1 aus Tab. 15. 
Tabelle 15.

Versuch XVI. 3. Juli 1913. Kaninchen, Gewicht 2150 g. $4^{3 / 4}$ Uhr $2 \mathrm{~g}$ Urethan subkutan. Begirn des Versuches 6 Uhr. Vagi intakt. Pulszahl in 10 Sek.: 43, Grösse der Pulswellen: $1 \mathrm{~mm} \mathrm{Hg}$, Blutdruck: $125 \mathrm{~mm} \mathrm{Hg}$.

\begin{tabular}{|c|c|c|c|c|c|c|c|}
\hline Nr. & Zeit & 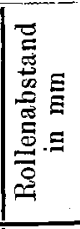 & 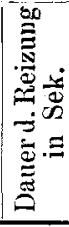 & 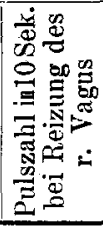 & 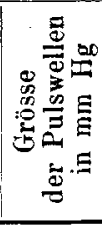 & 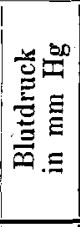 & Bemcrkungen \\
\hline 1 & $\begin{array}{ll}6^{\text {h }} & 15^{\prime} \\
6^{\text {h }} & 29^{\prime}\end{array}$ & 190 & 10 & 11 & $\begin{array}{c}30-41 \\
-\end{array}$ & $\overline{125}$ & $\begin{array}{l}10 \mathrm{ccm} \text { Jodthyreoglobulinlösung } \\
=0,2 \text { Substanz }=0,7 \mathrm{mg} \mathrm{J}, \\
\text { intravenös in } 2^{\prime} .\end{array}$ \\
\hline 2 & $\begin{array}{ll}6 \text { h } & 30 \\
6 \text { h } & 45^{\prime} \\
6 \text { h } & 46^{\prime}\end{array}$ & $\overline{190}$ & $\overline{10}$ & - & $\overline{41}$ & $\frac{125}{122}$ & \\
\hline
\end{tabular}

Die Pulszahl sank von 11 auf 1. Vgl. die Fig. 13 und 14.

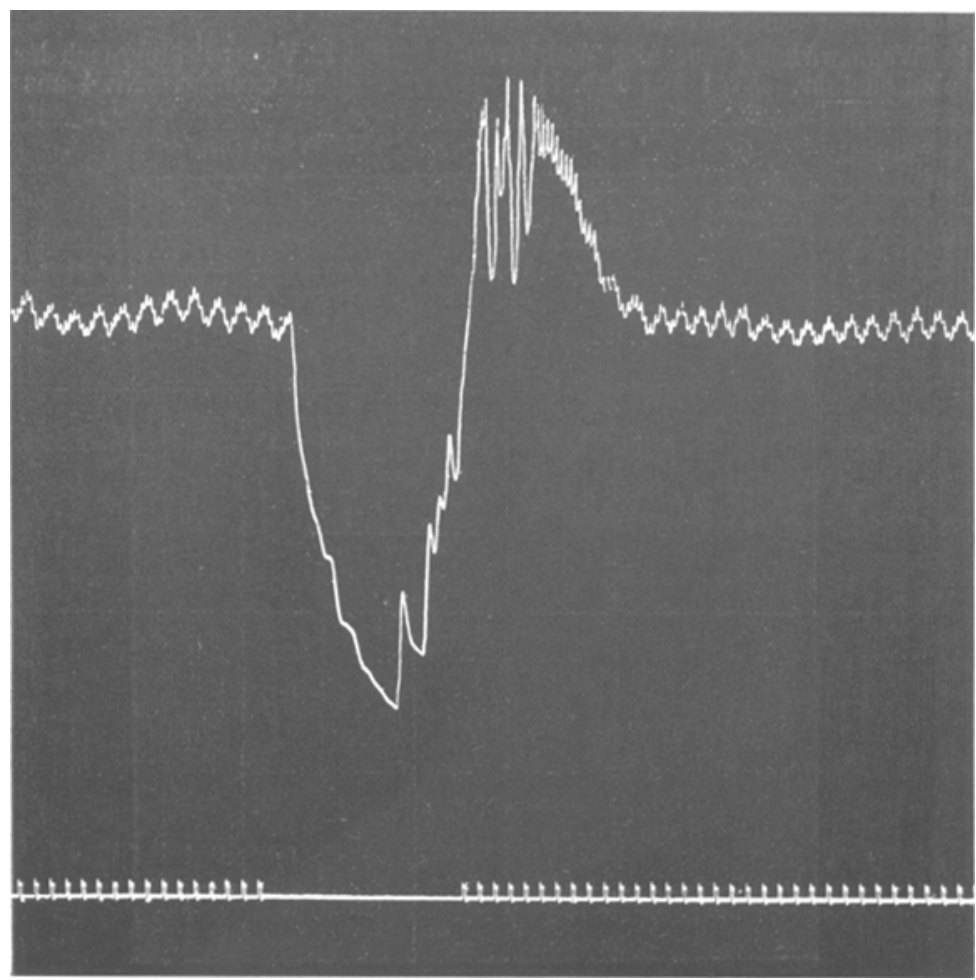

Fig. 14. Vaguswirkung nach der Jodthyreoglobulinzufuhr.

Nr. 2 aus Tab. 15. 
Folgende zwei Versuche beziehen sich auf die Katze.

Tabelle 16.

Versuch XLII. 10. März 1914. Katze, Gewicht 2900 g. $3^{1 / 2}$ Uhr $4 \mathrm{ccm}$ Paraldehyd. Während der: Dauer des Versuches Äther. Beginn des Versuches 4 Uhr 50 Min. Vagi intakt. Pulszahl in 10 Sek. : 31, Grösse der Pulswellen: $10 \mathrm{~mm} \mathrm{Hg}$, Blutdruck: $90 \mathrm{~mm} \mathrm{Hg}$.

\begin{tabular}{|c|c|c|c|c|c|c|c|c|}
\hline \multirow[t]{2}{*}{$\mathrm{Nr}$. } & \multirow[t]{2}{*}{ Zeit } & \multirow{2}{*}{ 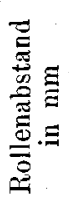 } & \multirow{2}{*}{ 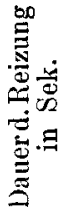 } & \multicolumn{2}{|c|}{$\begin{array}{c}\text { Depression } \\
\text { in } \mathrm{mm} \mathrm{Hg} \\
\text { während der } \\
\text { Reizung }\end{array}$} & \multirow{2}{*}{ 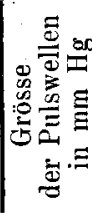 } & \multirow{2}{*}{ 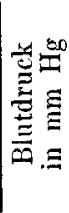 } & \multirow[t]{2}{*}{ Bemerktungen } \\
\hline & & & & $\begin{array}{l}\text { des } 1 . \\
\text { Vagus }\end{array}$ & $\begin{array}{l}\text { des } \mathbf{r} . \\
\text { Vagus }\end{array}$ & & & \\
\hline 1 & $5 \mathrm{~h} 17^{\prime}$ & 170 & 10 & 7 & - & 10 & - & \\
\hline 2 & 5 h $18^{\prime}$ & 160 & 10 & 16 & $\rightarrow$ & 10 & - & \\
\hline 3 & 5 h $24^{\prime}$ & 150 & 10 & 13 & - & 10 & - & \\
\hline 4 & $5 \mathrm{~h} 25^{\prime}$ & 150 & 10 & - & 20 & 10 & - & \\
\hline & $5^{\text {h }} 26^{\prime}$ & - & - & 一 & - & - & 82 & $\begin{array}{l}10 \mathrm{ccm} \text { Jodthyreoglobulin- } \\
\text { losung }(=0,2 \mathrm{~g} \text { Substanz } \\
=0,7 \mathrm{mg} J .) \text { intravenös } \\
\text { in } 1^{\prime} .\end{array}$ \\
\hline & 5 h $32^{\prime}$ & - & - & - & - & - & 96 & $10 \mathrm{ccm}$ der gleichen Lösung. \\
\hline & 5 h $38^{\prime}$ & $\overline{-\sigma}$ & - & - & - & - & 98 & $10 \mathrm{ccm}$ der gleichen Lösung. \\
\hline 5 & $5^{\text {h }} 34^{\prime}$ & 150 & 10 & 21 & $\overline{6}$ & 9 & - & \\
\hline 6 & $\begin{array}{l}5 \text { h } 36^{\prime} \\
5 \text { h } 40^{\prime}\end{array}$ & 150 & 10 & - & 30 & 12 & - & \\
\hline 7 & $5 \mathrm{~h} 42^{\prime}$ & 150 & 10 & - & 36 & 19 & $\ldots$ & \\
\hline 8 & $5^{\mathrm{h}} 44^{\prime}$ & 150 & 10 & 20 & - & 10 & - & \\
\hline 9 & $5^{\text {h }} 46^{\prime}$ & 150 & 10 & 24 & - & 10 & - & \\
\hline 10 & 5 h $50^{\prime}$ & 150 & 10 & - & 34 & 33 & 一 & \\
\hline 11 & 5 h $54^{\prime}$ & 150 & 10 & - & 48 & 37 & - & \\
\hline & $5 \mathrm{~b}$ & - & - & - & - & - & 80 & \\
\hline
\end{tabular}

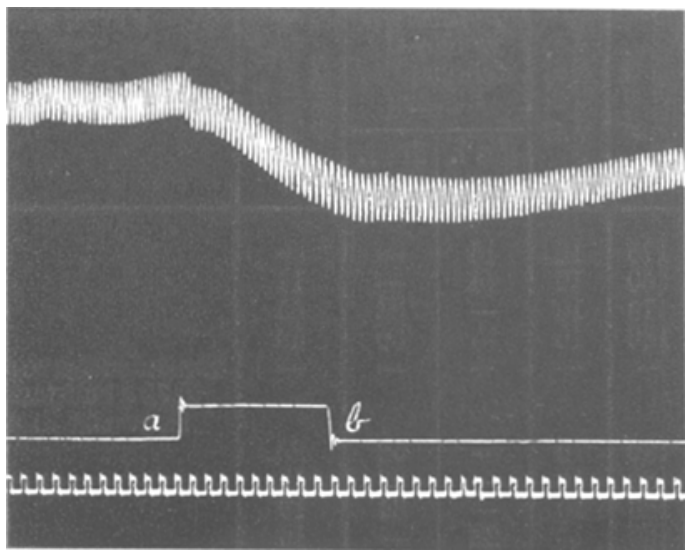

Fig. 15. Vaguswirkung vor der Jodtbyreoglobulinzufuhr. Nr. 4 aus Tab. 16. Von $a-b$ Dauer der Vagusreizung. 
Während bei einem Rollenabstand von 150 bei !Reizung des linken Vagus eine Depression von $13 \mathrm{~mm} \mathrm{Hg}$ und bei Reizung des rechten eine solche von

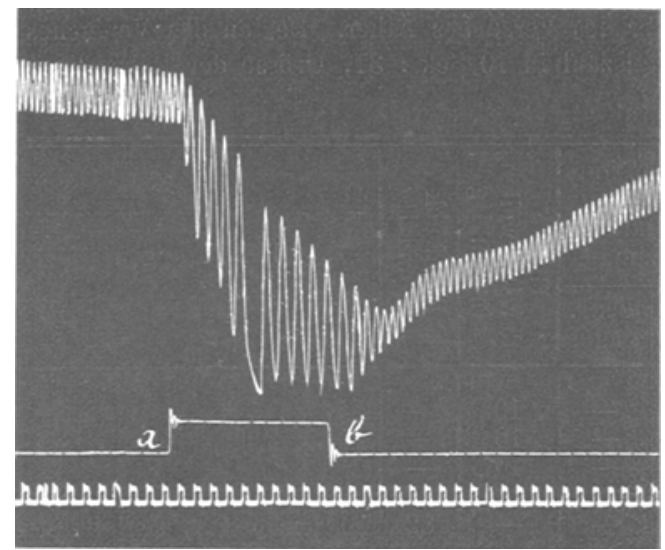

Fig. 16. Vaguswirkung nach'der Jodthyreoglobulinzufuhr. Nr, 11 aus Tab. 16. Von $a-b$ Dauer der Vagusreizung.
$20 \mathrm{~mm}$ eintritt, erfolgt nach Zufuhr von $0,6 \mathrm{~g}$ Jodthyreoglobulin eine solche von $20-24$ resp. 34-38. Dabei treten (unter entsprechender Verringerung der Zahl) Vaguspulse auf, welche vorher fehlten. Die Wirkung nimmt 10 Minuten nach der letzten Injektion noch zu. Fig. 15 und 16 geben zwei Kurvenaussehnitte wieder.

In einem zweiten Versuch an der Katze war das Resultat ein ähnliches:

Tabelle 17 .

Versuch XLV. 22. Mai 1914. Katze, Gewicht 2450 g. 4 Uhr $2,5 \mathrm{ccm}$ Paraldehyd subkutan. $\mathrm{O}_{2}$-Atmung. Äther. Vagi intakt. Blutdruck: $147 \mathrm{~mm} \mathrm{Hg}$, Pulszahl in 10 Sek.: 30, Grösse der Pulswellen: $10 \mathrm{~mm} \mathrm{Hg.}$

\begin{tabular}{|c|c|c|c|c|c|c|c|}
\hline \multirow[t]{2}{*}{$\mathrm{Nr}$. } & \multirow[t]{2}{*}{ Zeit } & \multirow{2}{*}{ 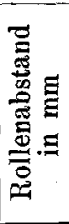 } & \multirow{2}{*}{ 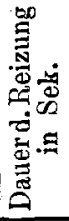 } & \multicolumn{2}{|c|}{$\begin{array}{c}\text { Depression } \\
\text { in mm Hg } \\
\text { während der } \\
\text { Reizung }\end{array}$} & \multirow{2}{*}{ 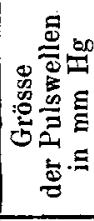 } & \multirow[t]{2}{*}{ Bemerkungen } \\
\hline & & & & $\begin{array}{l}\text { des } 1 . \\
\text { Vagus }\end{array}$ & $\begin{array}{l}\text { des } \mathrm{r} . \\
\text { Vagus }\end{array}$ & & \\
\hline 1 & $5 \mathrm{~h} 16^{\prime}$ & 175 & 10 & 15 & - & - & \\
\hline 2 & $5 \mathrm{~h} 20^{\prime}$ & 160 & 10 & 14 & 一 & 11 & \\
\hline 3 & 5 h $21^{\prime}$ & 175 & 10 & - & 84 & 55 & \\
\hline 4 & $\begin{array}{ll}5 \mathrm{~h} & 22 ! \\
5 \mathrm{~h} & 24\end{array}$ & 180 & 10 & 二 & 30 & 53 & $30 \mathrm{ccm}$ Jod thvreoglobulinlösung \\
\hline & & & & & & & $\begin{array}{l}(0,75 \mathrm{~g} \text { Substanz }=2,8 \mathrm{mg} \mathrm{J} .) \\
\text { intravenös in } 4^{\prime} .\end{array}$ \\
\hline 5 & $5^{\text {h }} 58^{\prime}$ & 180 & 10 & - & 52 & 28 & \\
\hline 6 & $5^{\text {h }} 59^{\prime}$ & 180 & 10 & - & 37 & 17 & \\
\hline 7 & $6^{\text {h }} 00^{\prime}$ & 180 & 10 & - & 34 & 19 & \\
\hline 8 & $6^{\text {h }} 01^{\prime}$ & 160 & 10 & 28 & - & 16 & \\
\hline 9 & $6^{\mathrm{h}} 02^{\prime}$ & 175 & 10 & 33 & - & 23 & \\
\hline 10 & $6^{\text {h }} \cdot 03^{\prime}$ & 175 & 10 & - & 31 & 23 & \\
\hline
\end{tabular}


Bei Rollenabstand 175 betrug die Depression vor der Jodthyreoglobulinzufuhr bei Reizung des linken Vagus $15 \mathrm{~mm} \mathrm{Hg}$, bei Reizung des rechten 34 , nach der Jodthyreoglobulinzufuhr 33 bzw. 31, bei Rollenabstand 160 betrug sie für

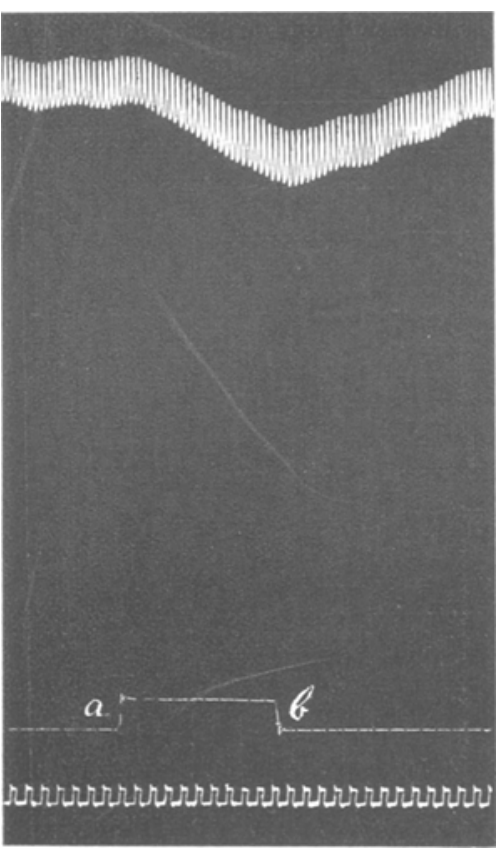

Fig. 17. Vagusreizung vor der Jodthyreoglobulinzufuhr. $\mathrm{Nr}$. 1 ans Tab. 17.

Von $a-b$ Dauer der Vagusreizung. den linken Vagus vorher 14, nachher 28, bei Abstand 180 für den rechten Vagus vorher 30, nachher $34-52$. Vgl. die Fig. 17 und 18.

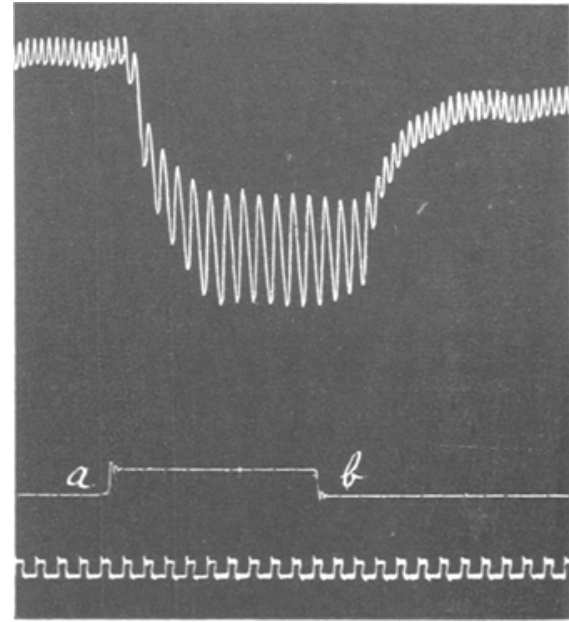

Fig. 18. Vagusreizung nach der Jodthyreoglobulinzufuhr. Nr. 9 aus Tab. 17 . Von $a-b$ Dauer der Vagusreizung.

Folgende Versuche wurden am $\mathrm{Hu}$ in angestellt.

Tabelle 18.

Versuch XLIV. 13. Mai 1914. Hund (Bulldogg), Gewicht $20 \mathrm{~kg}$. Vagi und Depressoren intakt.

\begin{tabular}{|c|c|c|c|c|c|c|c|c|}
\hline \multirow[t]{2}{*}{$\mathrm{Nr}$} & \multirow[t]{2}{*}{ Zeit } & \multirow{2}{*}{ 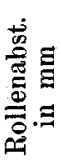 } & \multirow{2}{*}{ 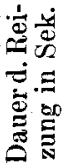 } & \multicolumn{2}{|c|}{$\begin{array}{c}\text { Pulszahl } \\
\text { wälirend d. } \\
\text { Reizung }\end{array}$} & \multirow{2}{*}{ 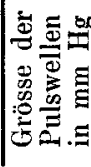 } & \multirow{2}{*}{ 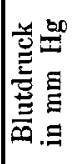 } & \multirow[t]{2}{*}{ Bemerkungen } \\
\hline & & & & $\begin{array}{l}\text { des } 1 . \\
\text { Vagus }\end{array}$ & $\begin{array}{l}\text { des } x \text {. } \\
\text { Vagus }\end{array}$ & & & \\
\hline \multirow{8}{*}{1} & $45^{\prime}$ & - & - & - & - & — & 160 & \multirow{8}{*}{$\begin{array}{l}10 \mathrm{ccm} \text { Jodthyreoglobulin } \\
\text { lösung (=0,25 g Substanz } \\
=0,9 \mathrm{mg} \text { J.). } \\
10 \mathrm{ccm} \text { derselben Lösung. } \\
10 \mathrm{ccm} \text { derselben Lösung. }\end{array}$} \\
\hline & $10 \mathrm{~h} 46^{\prime}$ & 165 & 10 & 21 & - & 54 & - & \\
\hline & $10^{\mathrm{h}} 51^{\prime}$ & 180 & 10 & - & 23 & 50 & - & \\
\hline & $10^{\mathrm{h}} 58^{\prime}$ & & - & - & - & - & 160 & \\
\hline & $10^{\text {h }} 59^{\prime} 30^{\prime \prime}$ & - & - & - & - & 一 & - & \\
\hline & $11 \mathrm{~b} 00^{\prime} 30^{\prime \prime}$ & $\overline{10 x}$ & $\overline{10}$ & $\overline{-}$ & - & 二 & - & \\
\hline & 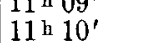 & $\begin{array}{l}16 \% \\
180\end{array}$ & $\begin{array}{l}10 \\
10\end{array}$ & 16 & - & 80 & - & \\
\hline & $11 \mathrm{~h} 11^{\prime}$ & 180 & 10 & - & 13 & 80 & - & \\
\hline
\end{tabular}


Vor der Jodthyreoglobulinzufuhr bewirkte die gleiche Reizung am rechten Vagus eine Abnahme der Pulsfrequenz auf 23 in 10 Minuten, nachher eine solche von 17 und 13, wobei die Grösse der Pulswellen von $50 \mathrm{~mm} \mathrm{Hg}$ auf 80 stieg. An linken Vagus betrug die Pulszahl vorher 21, nachher 16 und die Pulsgrösse stieg von 54 auf $87 \mathrm{~mm} \mathrm{Hg.}$ Vgl. Fig. 19 und Fig. 20.

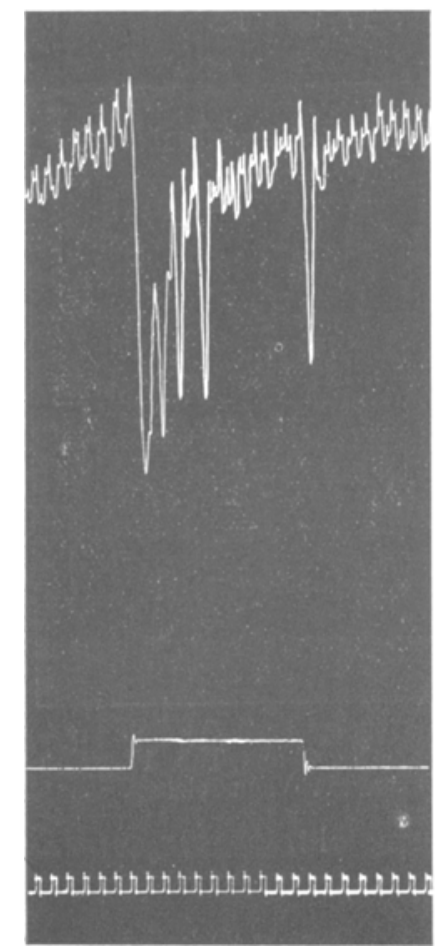

Fig. 19. Vagusreizung vor der Jodthyreoglobulinzufuhr. Nr. 2 aus Tab. 18.

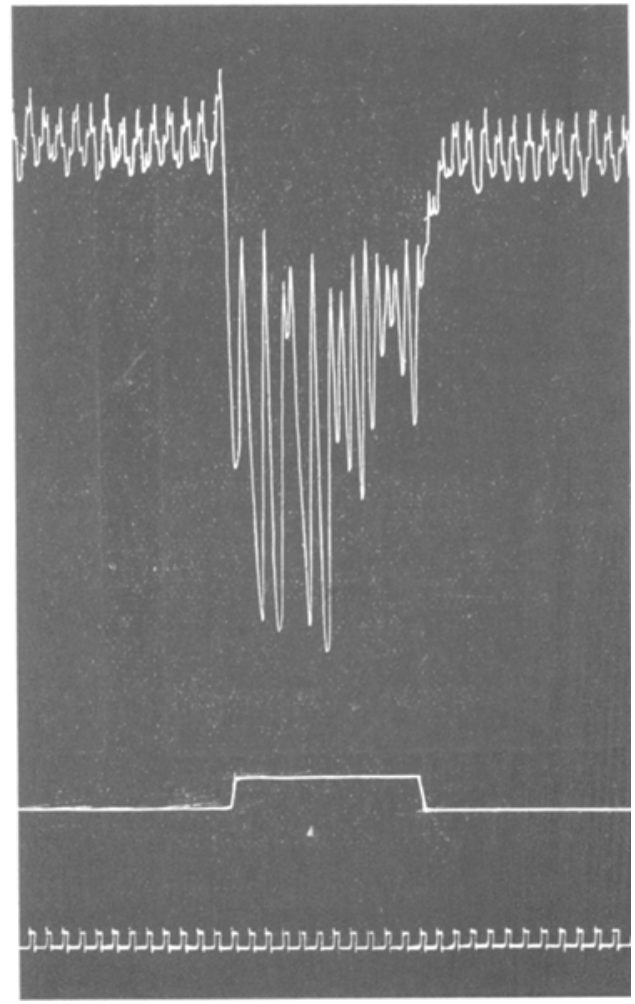

Fig. 20. Vagusreizung nach der Jodthyreoglobulinzufuhr. Nr. 5 aus Tab. 18.

Fassen wir das Resultat aller dieser Versuche zusammen, so ergibt sich, dass durchweg, bei Kaninchen, Hunden und Katzen, die intravenöse Zufuhr von $0,1-0,7$ g Jodthyreoglobulin (vom Hamme], Schwein und Hund) eine Zunahme der Ansprechbarkeit des Herzvagus wach sich zieht. Diese Wirkung tritt, was nicht unmittelbar aus den Tabellen hervorgeht, im Versuch aber oftmals nachgewiesen werden kounte, nicht gleich nach der Injoktion zutage, sondern deutlich erst nach vielleicht einer Minute und mebr. Oft 
konstatiert man eine Zunahme noch nach mehreren Minuten. Diese lange Latenzzeit ist wohl mit der kolloidalen Natur des Jodthyreoglobulins in Zusammenhang zu bringen. Die Wirkung hält lange Zeit an; ich habe sie noch nach mehr als einer Stunde, solang ich überhaupt den Versuch ausdehnte, nachweisen können; vermutlich hält sie noch viel länger an.

Die Ausschläge sind nicht immer so deutlich wie in den angeführten Versuchen, oft erweist sich der eine Vagus empfindlicher als der andere. Nie habe ich aber ein Tier gefunden, das gar nicht reagierte. Oft ist die Reaktion ausserordentlich stark.

v. Cy on ${ }^{1}$ ) gibt an, dass eine durch Atropin bewirkte Vaguslähmung durch Zufuhr von Jodothyrin aufgehoben wird. Diese Behauptung ist auf Widerspruch gestossen, indem darauf hingewiesen wurde, dass die Atropinwirkung spontan wieder ausklingt ${ }^{2}$ ), so dass, wenn danach eine zweite Substanz beigebracht werde, die Ansprechbarkeit zurückkehren könne, ohne dass diese Rückkehr dem Atropin zuzuschreiben sei. v. Fürth und $\mathrm{Schwarz}^{3}$ ) haben dann festgestellt, dass die Wiederansprechbarkeit nach Jodothyringebrauch nicht rascher eintritt als ohne Gebrauch desselben, und daraus den Schluss gezogen, dass Jod oth y r in auf die Vaguserregbarkeit keinen Einfluss habe. Da nun Jodthyreoglobulin in meinen Versuchen eine solche Wirkung kundgegeben hatte, stand zu prüfen, wie es sich gegenüber Atropin verhielt. Damit liess sich gleichzeitig die Richtigkeit der Schlussfolgerungen der beiden Autoren indirekt erproben. Die Versuche habe ich an Kaninchen angestellt.

Der Versuchsmodus war der, dass dem Versuchstier Atropin in Dosen von $1 \mathrm{mg}$ intravenös beigebracht wurde. Es geschah dies derart, dass eine Bürette mit einer wässerigen Lösung, welche in $1 \mathrm{ccm}$ $1 \mathrm{mg}$ Atropin enthielt, beschickt und mittels Glaskanüle und Gummischlauch mit der Vena jugularis verbunden wurde. Durch öffnen und Schliessen des Hahns wurde jeweilen $1 \mathrm{ccm}$ zufliessen gelassen. Es wurde die Zeit bestimmt, nach welcher der Vagus für einen faradischen

1) E. v. Cyon, Jodothyrin und Atropin. Dieses Arch. Bd. 70 3. 511. 1898. E. v. Cyon, Die physiologischen Herzgifte. Dieses Arch. Bd. 73 S. 42.1898.

2) E. Harnack, Über v. Cyon's antagonistische Versucbe mit JodothyrinAtropin. Zentralbl. f. Physiol. 1898 S. 291. - Vgl. auch B. v. Fennyvessy, Über die Wirkung des Schilddrüsensaftes auf die Zirkulation und Atmung nebst einem Anhang ubber Beziehungen zwischen Jodothyrin und Jodnatrium bzw. Atropin. Wiener klin. Wochenschr. 1900 S. 125 . - S. Is a a c und R. v. den Velden, Kreislaufwirkung jodierter Eiweisskörper. Verhandl. d. Kongr. f. inn. Med. 1907 S. 307 und S. Is a a c und R. v. den Velden, Untersuchungen über das Verhältnis des Kreislaufs bei Zufuhr jodierter Eiweisskörper. Med.-naturwissensch. Arch. Bd. 1 S. 105.1907.

3) loc. cit. 
Strom von gegebener Stärke, auf den er vor der Injektion deutlich ansprach, wieder erregbar war. Dabei wurde eine zu starke Stromstärke vermieden. Der Versuch lehrte, dass bei gleicher Dosis die Lähmungsdauer nach jeder Atropingabe dieselbe war. Als dies festgestellt war, wurde Jodthyreoglobulinlösung in eine Ohrvene injiziert und danach Atropin in gleicher Dosis zufliessen gelassen und wiederum die Zeit bestimmt, nach welcher die Ansprechbarkeit für den Strom auftrat Ich lasse ein Versuchsprotokoll folgen.

Tabelle 19.

Versuch XXV. 8. November 1913. Kan inchen, Gewicht $3480 \mathrm{~g} .10 \mathrm{Ubr}$. $2 \mathrm{~g}$ Urethan subkutan. Beginn des Versuches $11 \mathrm{Uhr}$. Vagi intakt. Grösse der Pulswellen: $2 \mathrm{~mm} \mathrm{Hg}$.

\begin{tabular}{|c|c|c|c|c|c|c|c|c|}
\hline \multirow[t]{2}{*}{$\mathrm{Nr}$. } & \multirow[t]{2}{*}{ Zeit } & \multirow{2}{*}{ 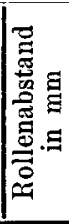 } & \multirow{2}{*}{ 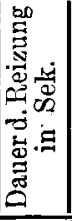 } & \multicolumn{2}{|c|}{$\begin{array}{l}\text { Pulszahl } \\
\text { während der } \\
\text { Reizung }\end{array}$} & \multirow{2}{*}{ 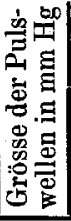 } & \multirow{2}{*}{ 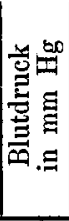 } & \multirow{2}{*}{ Bemerkungen } \\
\hline & & & & $\begin{array}{l}\text { des } \\
\text { 1. Vagus }\end{array}$ & $\begin{array}{l}\text { des } \\
\text { r. Vagus }\end{array}$ & & & \\
\hline \multirow{35}{*}{1} & $11^{\mathrm{h}} 14^{\prime}$ & 190 & 3 & - & 1 & 61 & - & \multirow{22}{*}{$\begin{array}{l}1 \mathrm{ccm} \text { Atropin (1 mg) } \\
\text { in } 11^{\prime \prime} \text { einlaufen } \\
\text { gelassen. }\end{array}$} \\
\hline & $11 \mathrm{~h} 15^{\prime}$ & 196 & 10 & - & 12 & 60 & - & \\
\hline & $11^{\mathrm{h}} 16^{\prime}$ & 180 & 2 & 2 & $\div$ & 52 & - & \\
\hline & $11^{\mathrm{h}} 17^{\prime}$ & 183 & 5 & 7 & - & 46 & - & \\
\hline & $11^{\text {h }} 18^{\prime}$ & 195 & 5 & 一 & 8 & 59 & - & \\
\hline & $11^{\text {h }} 19^{\prime}$ & - & - & - & - & - & 122 & \\
\hline & $11^{\mathrm{h}} 19^{\prime} 11^{\prime \prime}$ & - & - & - & 一 & - & 122 & \\
\hline & $11^{\text {h }} 19^{\prime} 20^{\prime \prime}$ & - & - & 一 & - & 一 & 120 & \\
\hline & $11^{\mathrm{h}} 19^{\prime} 40^{\prime \prime}$ & - & - & - & - & - & 116 & \\
\hline & $11^{\mathrm{h}} 19^{\prime} 50^{\prime \prime}$ & - & - & - & - & - & 119 & \\
\hline & $11^{\text {b }} 20^{\prime}$ & 183 & 5 & keineWirk. & - & - & 一 & \\
\hline & 11 h $20^{\prime} 26^{\prime \prime}$ & 195 & 5 & - & keine Wirk. & - & - & \\
\hline & $11^{\text {h } 21^{\prime} 17^{\prime \prime}}$ & 183 & 5 & keineWirk. & - & - & - & \\
\hline & $1^{\prime} 46^{\prime \prime}$ & 195 & 5 & - & 8 & 26 & - & \\
\hline & 11 h $22^{\prime} 14^{\prime \prime}$ & 183 & 5 & keineWirk. & - & - & - & \\
\hline & $11 \mathrm{~h} 23^{\prime} 01^{\prime \prime}$ & 195 & 5 & - & 6 & 31 & - & \\
\hline & $11^{\mathrm{h}} 23^{\prime} 54^{\prime \prime}$ & 183 & 5 & 17 & - & 16 & - & \\
\hline & $41^{\prime \prime}$ & 195 & 5 & - & 6 & 58 & - & \\
\hline & $29^{\prime \prime}$ & 183 & 5 & 13 & - & 14 & - & \\
\hline & $11 \mathrm{~h} 26^{\prime} 23^{\prime \prime}$ & 195 & 5 & - & 5 & 68 & - & \\
\hline & 11 & 183 & 5 & 10 & - & 15 & - & \\
\hline & $11 \mathrm{~h} 30^{\prime}$ & 183 & 5 & 9 & - & 46 & - & \\
\hline & 11 ь $30^{\prime} 30^{\prime \prime}$ & - & - & - & - & - & - & $1 \mathrm{mg}$ Atropin in $12^{\prime \prime}$. \\
\hline & 11 b $33^{\prime}$ & 195 & 5. & - & keineWirk. & - & - & \\
\hline & 11 h $33^{\prime} 57^{\prime \prime}$ & 183 & 5 & keineWirk. & - & - & -- & \\
\hline & 11 н $34^{\prime} 42^{\prime \prime}$ & 195 & 5 & 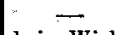 & 16 & 18 & - & \\
\hline & 11 h $34^{\prime} 53^{\prime \prime}$ & 183 & 5 & keineWirk. & - & - & - & \\
\hline & 11 h $36^{\prime} 22^{\prime \prime}$ & 195 & 5 & - & 8 & 34 & - & \\
\hline & $11^{\text {h }} 36^{\prime} 54^{\prime \prime}$ & 183 & 5 & keine Wirk. & - & -- & - & \\
\hline & $11^{\text {म }} 37^{\prime} 45^{\prime \prime}$ & 195 & 5 & - & 9 & 28 & - & \\
\hline & 11 h $39^{\prime}$ & 183 & 5 & 15 & - & 17 & - & \\
\hline & 11 h $40^{\prime}$ & 183 & 5 & 15 & - & 27 & - & \\
\hline & $11^{\mathrm{h}} 43^{\prime}$ & & - & - & - & - & - & $\begin{array}{c}10 \mathrm{ccm} \text { Jodthyreo- } \\
\text { globulinlösung }= \\
0=\end{array}$ \\
\hline & 11 h $47^{\prime} 30^{\prime \prime}$ & 195 & 5 & - & 8 & 44 & - & $0,7 \mathrm{mg} \mathrm{J}$.) intravenös \\
\hline & & 183 & 5 & 8 & & 38 & - & in $1^{\prime} 15^{\prime \prime}$ \\
\hline
\end{tabular}


Tabelle 19 (Fortsetzung).

\begin{tabular}{|c|c|c|c|c|c|c|c|c|}
\hline \multirow[t]{2}{*}{$\mathrm{Nr}$} & \multirow[t]{2}{*}{ Zeit } & \multirow{2}{*}{ 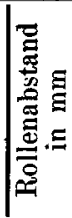 } & \multirow{2}{*}{ 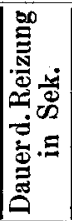 } & \multicolumn{2}{|c|}{$\begin{array}{l}\text { Pulszahl } \\
\text { während der } \\
\text { Reizung }\end{array}$} & \multirow{2}{*}{ 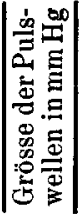 } & \multirow{2}{*}{ 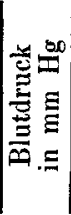 } & \multirow{2}{*}{ Bemerkungen } \\
\hline & & & & $\begin{array}{l}\text { des } \\
\text { 1. Vagus }\end{array}$ & $\begin{array}{l}\text { des } \\
\text { r. Vagus }\end{array}$ & & & \\
\hline \multirow[t]{24}{*}{3} & $11^{\text {h }} 49^{\prime}$ & - & - & - & - & & 144 & \multirow{24}{*}{$1 \mathrm{mg}$ Atropin in $12^{\prime \prime}$. } \\
\hline & 11 h $49^{\prime} 10^{\prime \prime}$ & - & 一 & $\ldots$ & - & - & 144 & \\
\hline & $11^{\text {h }} 49^{\prime} 20^{\prime \prime}$ & - & - & - & - & - & 133 & \\
\hline & $11^{\text {h }} 49^{\prime} 40^{\prime \prime}$ & - & 一 & -. & - & - & 138 & \\
\hline & $11^{\mathrm{h}} 49^{\prime} 50^{\prime \prime}$ & - & - & - & - & - & 140 & \\
\hline & $11^{\text {h }} 49^{\prime} 56^{\prime \prime}$ & 195 & 5 & - & keine Wirk. & - & - & \\
\hline & $11^{\text {h }} 50^{\prime} 07^{\prime \prime}$ & 183 & 5 & KeineW Wrk. & - & - & $1-$ & \\
\hline & $11^{\text {h }} 51^{\prime}$ & 195 & 5 & - & keineWirk. & - & - & \\
\hline & $11^{\text {h }} 51^{\prime} 30^{\prime \prime}$ & 183 & 5 & keineWirk. & - & - & - & \\
\hline & $11^{\text {h }} 52^{\prime}$ & 195 & 5 & - & keinew irk. & - & - & \\
\hline & $11 \mathrm{~h} 52^{\prime} 30^{\prime \prime}$ & 188 & 5 & keineWirk. & - & - & - & \\
\hline & $11^{\text {h }} 53^{\prime}$ & 195 & 5 & - & 10 & 36 & - & \\
\hline & $11^{\mathrm{h}} 53^{\prime} 22^{\prime \prime}$ & 183 & 5 & keineWirk. & - & - & - & \\
\hline & $11^{\text {h }} 53^{\prime} 43^{\prime \prime}$ & 195 & 5 & - & 10 & 30 & - & \\
\hline & $11^{\text {h }} 54^{\prime} 03^{\prime \prime}$ & 183 & 5 & keine Wirk. & - & - & - & \\
\hline & $11^{\mathrm{h}} 54^{\prime} 23^{\prime \prime}$ & 195 & 5 & - & 10 & 31 & - & \\
\hline & $11^{\text {' }} 54^{\prime} 55^{\prime \prime}$ & 183 & 5 & 17 & - & - & - & \\
\hline & $11^{\mathrm{h}} 56^{\prime}$ & 195 & 5 & - & 7 & 29 & - & \\
\hline & $11^{\text {h }} 56^{\prime} 20^{\prime \prime}$ & 183 & 5 & 13 & - & 20 & - & \\
\hline & $11^{\text {h }} 56^{\prime} 54^{\prime \prime}$ & 183 & 5 & 14 & - & 19 & - & \\
\hline & $11^{\text {h }} 58^{\prime}$ & 183 & 5 & 9 & - & 22 & - & \\
\hline & $11^{\text {h }} 58^{\prime} 50^{\prime \prime}$ & - & 一 & - & - & - & 136 & \\
\hline & 11 h $59^{\prime}$ & - & 一 & - & - & - & 一 & \\
\hline & $11^{\mathrm{h}} 59^{\prime} 20^{\prime \prime}$ & - & $\longrightarrow$ & - & 一 & - & 144 & \\
\hline \multirow{14}{*}{4} & $12^{\mathrm{h}} 01^{\prime} 30^{\prime \prime}$ & - & - & - & - & $\ldots$ & - & \multirow{14}{*}{$1 \mathrm{mg}$ Atropin in $12^{\prime \prime}$. } \\
\hline & $12^{\mathrm{h}} 02^{\prime}$ & & - & - & - & - & 134 & \\
\hline & $12^{\mathrm{h}} 02^{\prime} 05^{\prime \prime}$ & 195 & 5 & - & keineWirk. & - & - & \\
\hline & $12^{\mathrm{h}} 02^{\prime} 28^{\prime \prime}$ & 183 & 5 & keineWirk. & - & - & - & \\
\hline & $12 \mathrm{~h} 03^{\prime} 05^{\prime \prime}$ & 195 & 5 & - & keineWirk. & - & - & \\
\hline & $12^{\mathrm{h}} 03^{\prime} 27^{\prime \prime}$ & 183 & 5 & keineWirk. & - & - & - & \\
\hline & $12^{\mathrm{h}} 03^{\prime} 50^{\prime \prime}$ & 195 & 5 & - & 15 & 19 & - & \\
\hline & $12^{\mathrm{h} 04^{\prime}} 15^{\prime \prime}$ & 183 & 5 & keineWirk. & $\rightarrow$ & - & - & \\
\hline & $12^{\mathrm{h}} 04^{\prime} 38^{\prime \prime}$ & 195 & 5 & - & 12 & 32 & - & \\
\hline & $12^{\mathrm{h}} 05^{\prime} 15^{\prime \prime}$ & 195 & .5 & - & 8 & 27 & - & \\
\hline & $12 \mathrm{~h} 05^{\prime} 38^{\prime \prime}$ & 183 & 5 & keineWirk. & - & - & - & \\
\hline & $12^{\mathrm{h}} 06^{\prime} 04^{\prime \prime}$ & 195 & 5 & - & 9 & 33 & - & \\
\hline & $12^{\text {h }} 06^{\prime} 27^{\prime \prime}$ & 183 & 5 & 16 & - & - & - & \\
\hline & $12^{\mathrm{h}} 10^{\prime} 50^{\prime \prime}$ & - & - & - & - & - & 144 & \\
\hline \multirow[t]{13}{*}{5} & $12^{\mathrm{h}} 11^{\prime}$ & - & - & - & - & - & - & \multirow[t]{13}{*}{$2 \mathrm{mg}$ Atropin in $18 "$. } \\
\hline & $12^{\mathrm{h}} 11^{\prime} 30^{\prime \prime}$ & - & - & - & - & - & 132 & \\
\hline & $12^{\mathrm{h}} 11^{\prime} 50^{\prime \prime}$ & - & - & - & - & - & 136 & \\
\hline & $12^{\mathrm{h}} 12^{\prime}$ & 183 & 5 & keine Wirk. & - & - & - & \\
\hline & $12^{\text {h }} 12^{\prime} 15^{\prime \prime}$ & 195 & 5 & - & keineWir: & - & - & \\
\hline & $12^{\mathrm{h}} 12^{\prime} 43^{\prime \prime}$ & 175 & 5 & keine Wirk. & - & - & - & \\
\hline & $12^{\mathrm{h}} 13^{\prime} 18^{\prime \prime}$ & 180 & 5 & - & keineWirk. & - & 一 & \\
\hline & $12^{\mathrm{h}} 13^{\prime} 44^{\prime \prime}$ & 175 & 5 & keineWirk. & - & - & $\ldots$ & \\
\hline & $12^{\mathrm{h}} 14^{\prime} 15^{\prime \prime}$ & 175 & 5 & - & 14 & 8 & 一 & \\
\hline & $12^{\mathrm{h}} 14^{\prime} 29^{\prime \prime}$ & 175 & 5 & keineWirk. & 一 & - & 一 & \\
\hline & $12^{\mathrm{h}} 15^{\prime} 12^{\prime \prime}$ & 190 & 5 & - & 13 & 27 & - & \\
\hline & $12^{\mathrm{h}} 15^{\prime} 40^{\prime \prime}$ & 180 & 5 & keineWirk. & - & - & - & \\
\hline & $12^{\mathrm{b}} 16^{\prime} 06^{\prime \prime}$ & 190 & 5 & - & 10 & 33 & - & \\
\hline
\end{tabular}


Tabelle 19 (Fortsetzung).

\begin{tabular}{|c|c|c|c|c|c|c|c|c|}
\hline \multirow[t]{2}{*}{ Nr. } & \multirow[t]{2}{*}{ Zeit } & \multirow{2}{*}{ 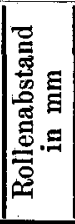 } & \multirow{2}{*}{ 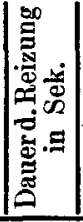 } & \multicolumn{2}{|c|}{$\begin{array}{c}\text { Pulszahl } \\
\text { während der } \\
\text { Reizung }\end{array}$} & \multirow{2}{*}{ 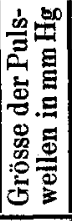 } & \multirow{2}{*}{ 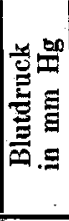 } & \multirow{2}{*}{ Bemerkungen } \\
\hline & & & & 1. Vagus & $\begin{array}{l}\text { des } \\
\text { r. Vagus }\end{array}$ & & & \\
\hline 6 & 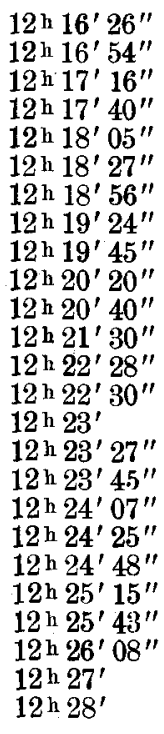 & $\begin{array}{l}180 \\
195 \\
183 \\
195 \\
183 \\
195 \\
183 \\
195 \\
183 \\
183 \\
- \\
- \\
- \\
180 \\
175 \\
180 \\
175 \\
180 \\
175 \\
180 \\
175 \\
195 \\
175 \\
175 \\
175\end{array}$ & $\begin{array}{r}5 \\
5 \\
5 \\
5 \\
5 \\
5 \\
5 \\
5 \\
5 \\
5 \\
- \\
- \\
5 \\
5 \\
5 \\
5 \\
5 \\
5 \\
5 \\
5 \\
5 \\
5 \\
5 \\
5\end{array}$ & $\begin{array}{c}\text { keineWirk. } \\
\text { keineWirk. } \\
\text { keineWirk. } \\
\overline{15} \\
\overline{9} \\
\overline{12} \\
\overline{-} \\
\overline{-} \\
\text { keineWirk. } \\
\text { keineWirk. } \\
\text { keineWirk. } \\
\text { keineWirk. } \\
\text { keineWirk. } \\
\text { keineWirk. } \\
16\end{array}$ & $\begin{array}{c}\overline{13} \\
\frac{8}{7} \\
\overline{6} \\
\overline{-} \\
\overline{-} \\
\overline{-} \\
\text { keinewirk. } \\
\overline{15} \\
\overline{12} \\
\overline{12} \\
\overline{11} \\
\overline{-} \\
-\end{array}$ & $\begin{array}{l}\overline{26} \\
\frac{38}{-38} \\
46 \\
7 \\
50 \\
30 \\
18 \\
= \\
= \\
= \\
= \\
\overline{20} \\
\overline{24} \\
\overline{31} \\
= \\
-\end{array}$ & $\begin{array}{l}= \\
= \\
= \\
= \\
= \\
= \\
\overline{116} \\
\overline{110} \\
= \\
= \\
= \\
= \\
\bar{Z} \\
= \\
=\end{array}$ & $1 \mathrm{mg}$ Atropin in $15^{\prime \prime}$. \\
\hline
\end{tabular}

Der Übersichtlichkeit halber soll das Resultat tabellarisch zusammengestellt sein.

Die Ansprechbarkeit kehrt zurück:

V or der Jodthyreoglobulinzufuhr

nach der ersten Atropingabe (1 mg)

für den 1. Vagus für den $\mathrm{r}$. Vagus andeutungsweise . . . nach $4^{\prime} 54^{\prime \prime}$ nach $2^{\prime} 46^{\prime \prime}$ voll ....... . " $11^{\prime} \quad " 5^{\prime} 41^{\prime \prime}$

nach der zweiten Atropingabe $(1 \mathrm{mg})$ andeutungsweise . . . nach $8^{\prime} 30^{\prime \prime} \quad$ " $4^{\prime} 12^{\prime \prime}$ nicht ganz voll . . . " $9^{\prime} 30^{\prime \prime} " 7^{\prime} 15^{\prime \prime}$

$\mathrm{N}$ a ch der Jodthyreoglobulinzufuhr

nach der dritten Atropingabe $(1 \mathrm{mg})$ andeutungsweise . . . nach $5^{\prime} 55^{\prime \prime} \quad, 4^{\prime}$ voll

nach der vierten Atropingabe $(1 \mathrm{mg})$ andeutungsweise . . . nach 4'57" "2'20" voll . . . . . . . 
nach der fünften Atropingabe $(2 \mathrm{mg})$

für den l. Vagus für den r. Vagus

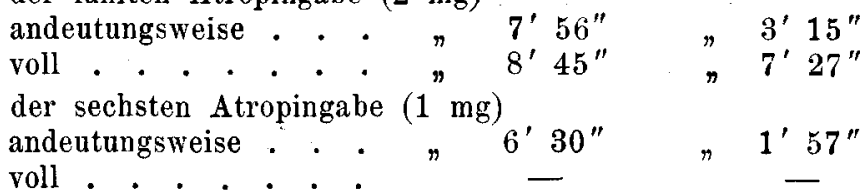
gekehrt :

In einem zweiten Versuch war die volle Ansprechbarkeit zuräckV or der Jodthyreoglobulinzufuhr

nach der ersten Atropingabe (1 mg)

$$
\text { nach } 12^{\prime} \quad \text { nach } 21^{\prime} 30^{\prime \prime}
$$

nach der zweiten Atropingabe ( $1 \mathrm{mg}$ )

nach $12^{\prime} 30^{\prime} \quad$ " $19^{\prime}$

N a c h Jodthyreoglobulinzufuhr

für den 1. Vagus für den r. Vagus

nach $1 \mathrm{mg}$ Atropin

$$
\text { " } 14^{\prime} 30^{\prime} \quad \text { "20'30" }
$$

Eine Verkürzung der Lähmungsdauer ist somit nach Jodthyreoglobulinzufuhr nicht zu konstatieren. Es ist somit dieser Modus nicht geeignet, eine Wirkung desselben auf die Vaguserregbarkeit darzutun.

\section{Wirkung auf die Ansprechbarkeit des Depressors.}

Zur Prüfung dieser Frage wurden nur Kaninchen herangezogeu. Technisch ist $\mathrm{zu}$ dem im Abschnitt über Methodik Gesagten noch folgendes zu bemerken. Der $\mathrm{N}$. depressor wurde beiderseits am Hals frei präpariert, wo er als dünner Faden dicht neben dem Sympathikus verläuft. Die Präparierung muss sorgfältig geschehen und jede Zerrung vermieden werden, da der feine Nervenast leicht Schaden nimmt. Auch ist für sofortiges Zurückbetten in den Wundspalt und Zudecken desselben nach jeder Reizung Sorge zu tragen, um Eintrocknung zu verhüten. Beobachtet man nicht alle diese Kautelen, so erlebt man, dass die Ansprechbarkeit allmählich abnimmt und schliessljch sogar ganz erlischt. Es ist darum geraten, überhaupt möglichst wenig Reizungen vorzunehmen und sich auf das gerade Notwendige zu beschränken. Auch dann erlebt man noch oft genug Enttäuschungen. Versuche am Depressor des Kaninchens erfordern jedenfalls grosse Sorgfalt.

Der Nerv wurde abgebunden und zerschnitten.

Ich lasse zwei Protokollauszüge folgen, welche die deutlichsten Resultate unter meinen Versuchen wiedergeben. 
Tabelle 20.

Versuch XV. 26. Juni 1913. Kaninchen, Gewicht 2950 g. $4 \frac{1 / 2}{2}$ Uhr 2 g Urethan subkutan. Vagi und Depressoren intakt.

\begin{tabular}{|c|c|c|c|c|c|c|c|c|}
\hline $\mathrm{Nr}$. & Zeit & 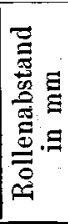 & 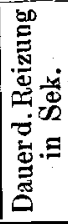 & 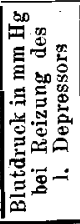 & 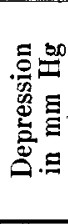 & 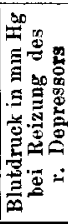 & 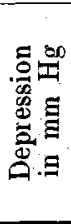 & Bemerkungen \\
\hline 1 & 5 h $50^{\prime}$ & 180 & 10 & - & - & 113 & 29 & \multirow{14}{*}{$\begin{array}{l}10 \mathrm{ccm} \text { Jodthyreoglobulin- } \\
\text { Iösung }(=\mathrm{ca} .0,2 \mathrm{~g} \text { Sub- } \\
\text { stanz }=0,7 \mathrm{mg} \text { J.) intra- } \\
\text { venös in } 1^{\prime} 5^{\prime \prime} \text {. }\end{array}$} \\
\hline & 5 h $50^{\prime} 10^{\prime \prime}$ & & 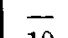 & $\overline{10}$ & - & 89 & & \\
\hline 2 & 5 h $51^{\prime}$ & 160 & 10. & $\begin{array}{r}112 \\
82\end{array}$ & 30 & 二 & - & \\
\hline & 5 h $51^{\prime} 20^{\prime \prime}$ & - & - & $\begin{array}{l}04 \\
110\end{array}$ & - & - & - & \\
\hline & $5^{\text {h }} 51^{\prime} 30^{\prime \prime}$ & - & - & 115 & - & - & - & \\
\hline & $5^{\mathrm{h}} 57^{\prime}$ & - & - & - & - & - & - & \\
\hline 3 & $5^{\text {h }} 57^{\prime} 12^{\prime \prime}$ & 180 & 10 & - & - & $\begin{array}{r}134 \\
98\end{array}$ & 36 & \\
\hline 4 & $6^{\mathrm{h}}$ & 160 & 10 . & 132 & 48 & - & - & \\
\hline & $6^{\text {h }} 00^{\prime} 13^{\prime \prime}$ & $\overline{180}$ & $\overline{10}$ & 84 & & $\overline{134}$ & 一 & \\
\hline & $\begin{array}{l}6^{\text {nh }} 04^{\prime} \\
6^{\circ} 04^{\prime \prime} 10^{\prime \prime}\end{array}$ & 180 & 10 & - & 一 & $\begin{array}{l}134 \\
104\end{array}$ & 30 & \\
\hline 5 & $6 \mathrm{~h} 07^{\prime}$ & 160 & 10 & 138 & 40 & 一 & - & \\
\hline 6 & $\begin{array}{l}6 \text { h } 07^{\prime} 12^{\prime \prime} \\
6 \text { h } 09^{\prime}\end{array}$ & $\overline{180}$ & 10 & $\frac{98}{-}$ & $\ldots$ & $\overline{136}$ & $\overline{0}$ & \\
\hline & $6^{\text {h }} 09^{\prime} 10^{\prime \prime}$ & & - & - & 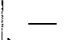 & 110 & 26 & \\
\hline 7 & $\begin{array}{l}6^{\text {h }} 18^{\prime} \\
6^{\text {h }} 18^{\prime \prime} 12^{\prime \prime}\end{array}$ & 160 & 10 & $\begin{array}{l}138 \\
102\end{array}$ & 36 & 二 & - & \\
\hline
\end{tabular}

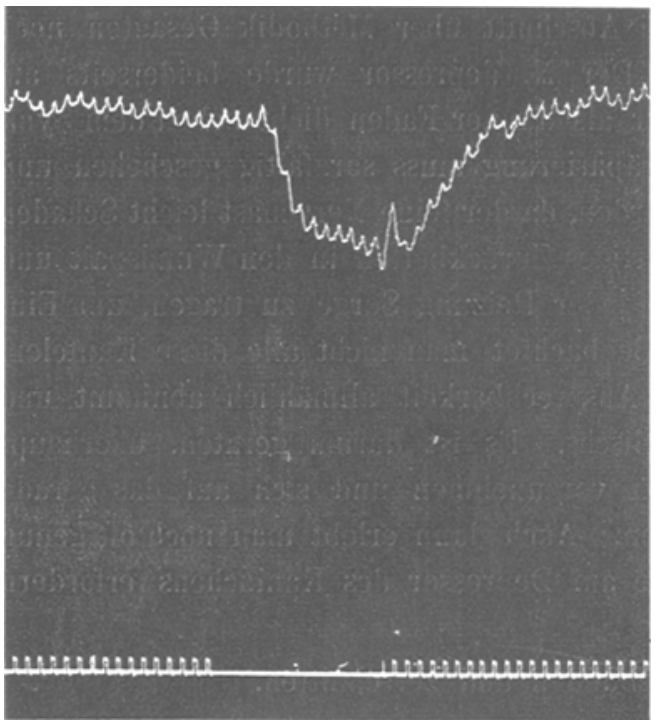

Fig. 21. Depressorreizung vor der Jodthyreoglobulinzufuhr. Nr. 2 aus Tab. 20.
Der Versuch ergibt. dass, während die Reizung des linken Depressors bei einem Rollenabstand von $160 \mathrm{~mm}$ eine Depression von $30 \mathrm{~mm} \mathrm{Hg}$ auslöste, sie nach der Jodthyreoglobulininjektion eine solche bis $48 \mathrm{~mm}$ bewirkte. Die Reizung des rechten Depressors ergab bei $180 \mathrm{~mm}$ Rollenabstand vor der Injektion eine Depression von $22 \mathrm{~mm}$ und nachher eine solche bis 36 . Fig 21 u. 22 geben zwei Ausschnitte aus der Kurve wieder. 


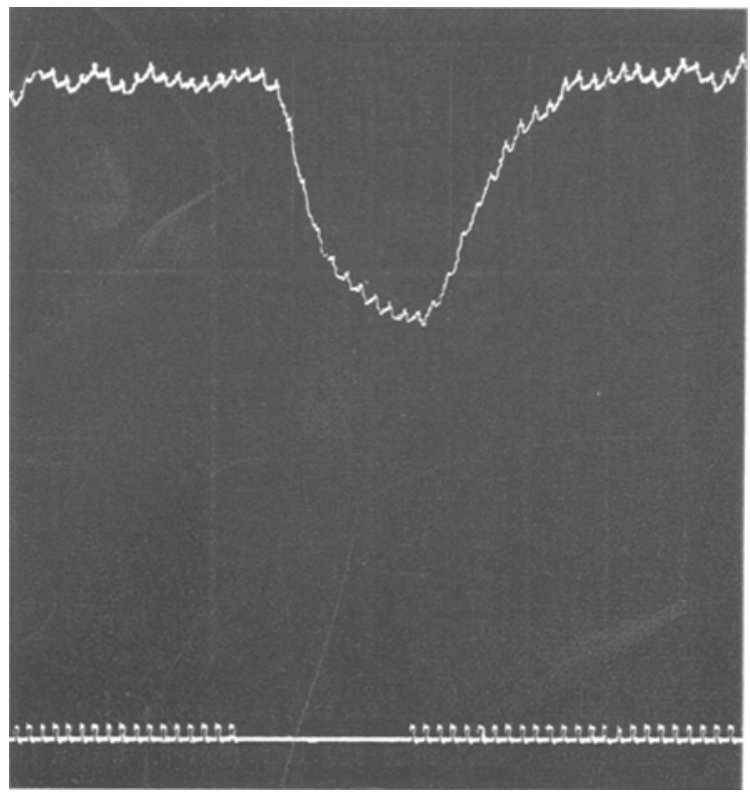

Fig. 22. Depressorreizung nach der Jodthyreoglobulinzufubr. Nr. 4 aus Tab. 20.

\section{Tabelle 21.}

Versuch XXX. Kaninchen, Gewicht 2800 g. $3^{3 / 4}$ Uhr 2 g Urethan subkutan. Beginn des Versuches 4 Uhr 45 Min.

\begin{tabular}{|c|c|c|c|c|c|c|c|c|}
\hline Nr. & Zeit & 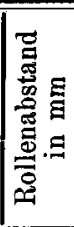 & 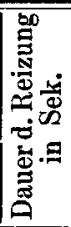 & 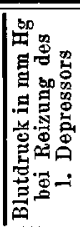 & 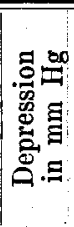 & 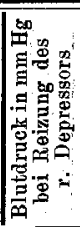 & 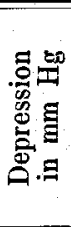 & Bemerkungen \\
\hline \multirow{3}{*}{1} & $5 \mathrm{~h} 01^{\prime}$ & 180 & 10 & - & - & 118 & 00 & \multirow{13}{*}{$\begin{array}{l}10 \mathrm{ccm} \text { Jodthyreoglobulin- } \\
\text { lösung }(=0,17 \mathrm{~g} \text { Substanz } \\
=0,6 \mathrm{mg} \text { J. }) \text { in } 1^{\prime} 4^{\prime \prime} \\
\text { intravenös. }\end{array}$} \\
\hline & $5 \mathrm{~h}^{\mathrm{h}} 1^{\prime} 10^{\prime \prime}$ & - & - & - & - & 98 & 20 & \\
\hline & $5 \mathrm{~h} 01^{\prime} 27^{\prime \prime}$ & 一 & - & $\rightarrow$ & - & 121 & 一 & \\
\hline 2 & $5 \mathrm{~h} 05^{\prime}$ & 195 & 10 & 126 & 22 & - & - & \\
\hline \multirow[t]{2}{*}{3} & $\begin{array}{l}5^{n} 05,12^{\prime \prime} \\
5 \text { h } 06^{\prime}\end{array}$ & $\overline{195}$ & $\overline{10}$ & 104 & - & $\overline{116}$ & $\overline{10}$ & \\
\hline & 5 h $06^{\prime} 12^{\prime \prime}$ & - & - & - & - & 98 & 18 & \\
\hline 4 & 5 h $10^{\prime}$ & 195 & 10 & 117 & 15 & - & - & \\
\hline 5 & $\begin{array}{l}5 \mathrm{~h} 10^{\prime} \\
5^{\mathrm{h}} 12^{\prime}\end{array}$ & 180 & 10 & 102 & - & $\overline{119}$ & $\overline{\mathrm{ar}}$ & \\
\hline & $5^{\text {h } 12^{\prime} 06^{\prime \prime}}$ & - & - & 一 & - & 98 & 21 & \\
\hline \multirow[t]{4}{*}{6} & $5^{\text {h }} 15^{\prime}$ & 180 & 10 & - & $=$ & $\begin{array}{r}107 \\
8 ?\end{array}$ & 25 & \\
\hline & 5 h $15^{\prime} 30^{\prime \prime}$ & $=$ & - & - & - & 118 & 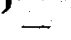 & \\
\hline & 5 h $20^{\prime}$ & - & - & $\ldots$ & - & 118 & - & \\
\hline & $5 \mathrm{~h} 21^{\prime}$ & - & - & - & - & 128 & - & \\
\hline & lüger's & fï & & & & & & 36 \\
\hline
\end{tabular}


Tabelle 21 (Fortsetzung).

\begin{tabular}{|c|c|c|c|c|c|c|c|c|}
\hline Nr. & Zeit & 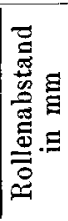 & 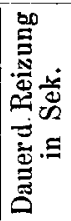 & 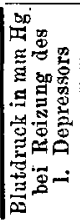 & 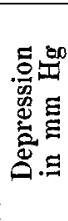 & 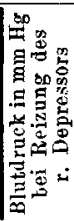 & 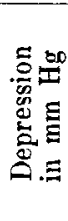 & Bemerkungen \\
\hline 7 & $\begin{array}{l}5 \text { h } 25^{\prime} \\
5 \text { h } 25^{\prime} 10^{\prime \prime}\end{array}$ & 180 & 10 & - & - & $\begin{array}{r}120 \\
82\end{array}$ & 41 & \\
\hline 8 & $\begin{array}{l}5 \text { h } 28^{\prime} \\
5 \text { h } 28^{\prime} 09^{\prime \prime}\end{array}$ & 195 & 10 & $\begin{array}{l}125 \\
111\end{array}$ & 14 & - & - & \\
\hline & $\begin{array}{l}5 \mathrm{~h} 35^{\prime} \\
5 \mathrm{~h} 36^{\prime}\end{array}$ & - & - & 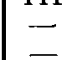 & - & $\begin{array}{l}130 \\
134\end{array}$ & - & 9 ccm derselben Jodthyreo- \\
\hline 9 & $\begin{array}{l}5 \text { h } 37^{\prime} \\
5 \text { h } 37^{\prime} 10^{\prime \prime}\end{array}$ & 195 & 10 & $\begin{array}{l}184 \\
108\end{array}$ & 26 & 二 & - & \\
\hline 10 & $\begin{array}{l}5 \text { h } 39^{\prime} \\
5 \text { h } 39^{\prime} 10^{\prime \prime}\end{array}$ & 195 & 10 & - & - & $\begin{array}{l}130 \\
106\end{array}$ & 24 & \\
\hline 11 & $\begin{array}{l}5 \text { h } 42^{\prime} \\
5 \text { h } 42^{\prime} 09^{\prime \prime}\end{array}$ & 195 & 10 & $\begin{array}{l}127 \\
101\end{array}$ & 26 & - & - & \\
\hline
\end{tabular}

Während die Reizung des linken Depressors bei Rollenabstand 195 eine Depression von $15-22 \mathrm{~mm} \mathrm{Hg}$ hervorrief, bewirkte sie nach rler Jodthyreoglobulinzufuhr eine solche von $26 \mathrm{~mm}$. Rechterseits machte sie bei Rollenabstand $19518 \mathrm{~mm} \mathrm{Hg}$ und bei Rollenabstand $180 \quad 20-25 \mathrm{~mm} \mathrm{Hg}$ aus; nach Jodthyreoglobulinzufuhr dagegen 24 bzw. $41 \mathrm{~mm} \mathrm{Hg.} \mathrm{Vgl.} \mathrm{die} \mathrm{Fig.} 23$ und 24 .

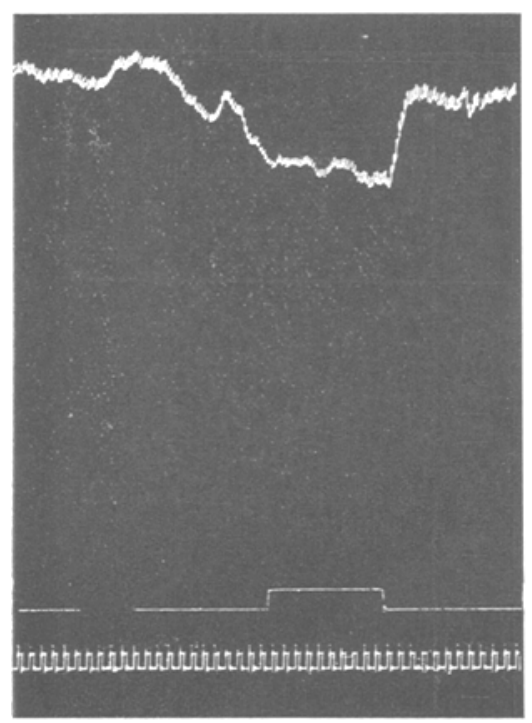

Fig. 23. Depressorreizung vor der Jodthyreoglobulinzufuhr. Nr.3 aus Tab. 21.

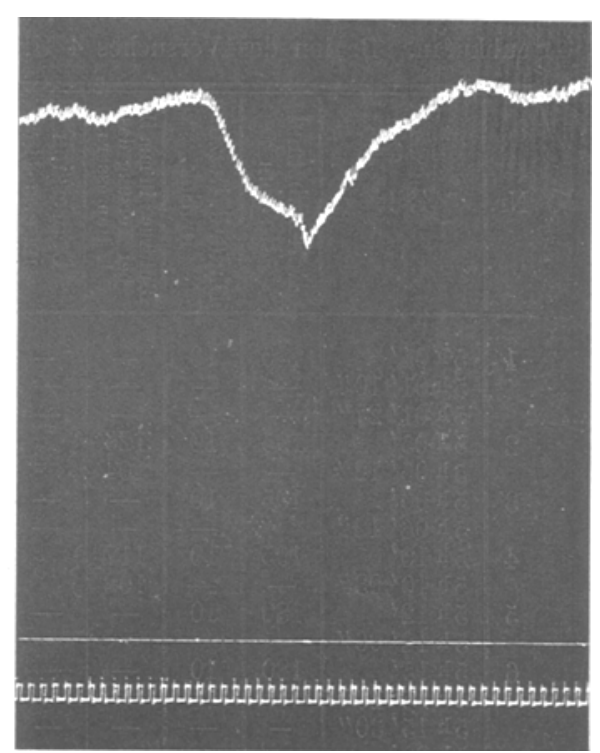

Fig. 24. Depressorreizung nach der Jodthyreoglobulinzufuhr. Nr. 7 aus Tab. 21. 


\section{Wirkung auf die Ansprechbarkeit des Splanchnikus.}

Es sollte nun geprüft werden, ob der dem sympathischen Apparat zugehörige Nerv durch Jodthyreoglobulin in gleicher Weise beeinflusst wird wie die bisher besprochenen. Das Ergebnis dieser Untersuchungen ist in folgendem niedergelegt.

Was die Methodik anbelangt, so wurde dem Tier (Kaninchen) in Seitenlage ein $5 \mathrm{~cm}$ langer Schnitt im 11. Interkostalraum gemacht und unter schonendem Vorgehen der Splanchnikus proximalwärts der Nebenniere aufgesucht. Der Nerv wurde auf einer Strecke von $1^{1 / 2} \mathrm{~cm}$ frei präpariert und auf die Schlinge genommen. Bei der Reizung wurde er sorgfältig etwas emporgehoben unter Vermeidung jeder Zerrung.

$$
\text { Tabelle } 22 .
$$

Versuch LVIII. 11. November 1915. Kan inchen, Gewicht 2400 g. $5 \mathrm{ccm} 5 \%$ iges Chloralhydrat per rectum eingeführt. $\mathrm{O}_{2}$-Atmung unter Zusatz von Äther.

\begin{tabular}{|c|c|c|c|c|c|c|c|}
\hline $\mathrm{Nr}$. & Zeit & 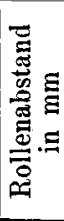 & 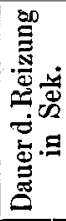 & 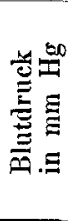 & $\begin{array}{c}\text { Reizung } \\
\text { des }\end{array}$ & 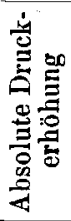 & Bemerkungen \\
\hline & $11^{\mathrm{h}} 04^{\prime}$ & & - & 46 & - & - & \multirow{14}{*}{$\begin{array}{l}0,15 \mathrm{~g} \text { Jodthyreoglobulin } \\
\text { in } 35^{\prime \prime} \text {. } \\
0,15 \mathrm{~g} \mathrm{Jodthyreoglobulin} \\
\text { in } 25^{\prime \prime} \text {. }\end{array}$} \\
\hline 1 & 11 h $05^{\prime}$ & 30 & 5 & 46 & 1. Splanchn. & 24 & \\
\hline & $11^{\text {h }} 05^{\prime} 06^{\prime \prime}$ & 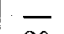 & - & 70 & - & 24 & \\
\hline 2 & $11^{\text {h }} 05^{\prime} 30^{\prime \prime}$ & 80 & 5 & 48 & 1. Splanchn. & 22 & \\
\hline & $\begin{array}{l}11 \mathrm{~h} 05^{\prime} 36^{\prime \prime} \\
11^{\mathrm{h} 06^{\prime} 08^{\prime \prime}}\end{array}$ & $\overline{-}$ & $\overline{-}$ & 48 & 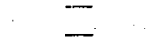 & - & \\
\hline 3 & 11 h $07^{\prime}$ & 30 & 5 & 46 & r. Splanchn. & 99 & \\
\hline & $11^{\text {h }} 07^{\prime} 08^{\prime \prime}$ & - & - & 68 & - & 28 & \\
\hline & $\begin{array}{l}11^{\text {h }} 09^{\prime} \\
11^{\text {h }} 09^{\prime} 35^{\prime \prime}\end{array}$ & 二 & 二 & $\begin{array}{l}47 \\
56\end{array}$ & 二 & - & \\
\hline & $11 \mathrm{~h} 10^{\prime}$ & 一 & - & 50 & - & - & \\
\hline$A$ & $11^{\text {h }} 10^{\prime} 25^{\prime \prime}$ & $\overline{6}$ & - & 53 & - & - & \\
\hline 4 & $\begin{array}{l}11 \mathrm{hl} 14^{\prime} \\
11 \mathrm{~h} 14^{\prime} 08^{\prime \prime}\end{array}$ & 30 & 5 & $\begin{array}{l}42 \\
74\end{array}$ & 1. Splanchn. & 32 & \\
\hline 5 & 11 h $17^{\prime}$ & 30 & 5 & 43 & 1. Splanchn. & 30 & \\
\hline e & $11^{\text {h }} 17^{\prime} 08^{\prime \prime}$ & $\rightarrow$ & - & 73 & - & 00 & \\
\hline b & $\begin{array}{l}11^{\text {h }} 20^{\prime} \\
11^{\prime} 20^{\prime \prime} 08^{\prime \prime}\end{array}$ & 30 & -5 & $\begin{array}{l}43 \\
71\end{array}$ & r. Splanchn. & 28 & \\
\hline
\end{tabular}

Der Versuch zeigt, dass J od th yre og lobulin die A n sprechbarkeit des Splanchnikus gegenüber dem faradischen Strom fördert. Die Druckerhöhung betrug nach der Jodthyreoglobulinzufuhr rund $30 \%$ mehr als vorher. 
Die Fig. 25 und 26 geben den Effekt der Reizung Nr. 2 und 4 aus Tabelle 1 bildlich wieder.

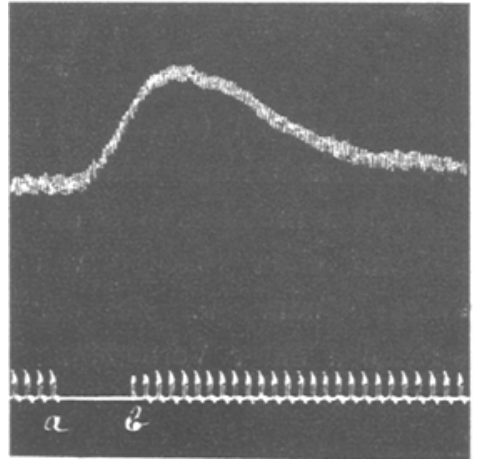

Fig. 25. Reizung des Splanchnikus vor Jodthyreoglobulinzufulhr. Nr. 3 aus Tab. 22. Von $a-b$ Dauer der Reizung.

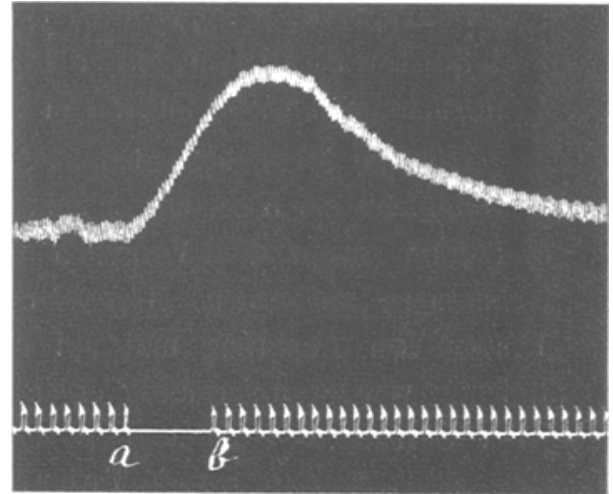

Fig. 26. Reizung des Splanchnikus nach Jodthyreoglobulinzufuhr. Nr. 4 aus Tab. 22. Von $a-b$ Dauer der Reizung.

\section{Wirkung auf den Adrenalineffekt.}

Kraus und Friedenthal ${ }^{1}$ ) haben gezeigt, dass Schilddrusenextrakte die pressorische Wirkung des Adrenalins verstärken. E's handelt sich darum, zu wissen, ob diese Eigenschaft dem Jodthyreoglobulin zukommt.

Nach verschiedenen, nicht zum Ziele führenden Vorversuchen erwies sich folgender Modus als der geeignetste. Es wurde dem in Urethanwarkose aufgespannten Tier durch die Vena jugularis innerhalb einer bestimmten Zeit eine bestimmte Menge einer sehr verdünnten Adrenalin- (resp. Suprarenin-) lösung (0,001 g Subst. in $50 \mathrm{ccm}$ physiol. Kochsalzlösung) eingeführt. Beide Substanzen wurden mir in liberaler Weise von den resp. Herstellern der Präparate, Parke, Davis \& Co. und Meister \& Lucius in Höchst, zur Verfügung gestellt. Ich spreche beiden Firmen auch an dieser Stelle meinen Dank aus. Wenn die Zufuhr der Substanz im gleichen Zeitabschnitt geschieht, so erhält man jedesmal die gleiche Blutdruckerhöhung, was sowohl die Intensität wie die Dauer anbelangt. Durch Verwendung einer Bürette mit Glashahn und einer im Gummischlauch eingeschalteten Glaskapillare kann man auf die Sekunde genau den Einlauf regulieren. Der Glashahn hat gegenüber dem Quetschhahn

1) loc. cit. 
den Vorteil, dass man ihn rasch vollständig öffnen und schliessen kann. Meist wurde $1 \mathrm{ccm}$ Lösung einlaufen lassen. Um Vergleichswerte zu erhalten, ist es durchaus erforderlich, diese Kautele einzuhalten, da sonst sich Differenzen in den Kurvenbildern ergeben. Zu den Versuchen wurden Kaninchen, Hunde und Katzen verwendet.

Ich lasse die Protokolle folgen.

\section{Tabelle 23.}

Versuch XIII. 19. Juni 1913. Kan inchen, Gewicht $2750 \mathrm{~g}$. 4 Uhr $2 \mathrm{~g}$ Urethan subkutan. Beginn des Versuches 5 Jhr 20 Min. Vagi und Depressores intakt. Pulszahl in 10 Sek.: 44, Grösse der Pulswellen: $1 \mathrm{~mm} \mathrm{Hg}$. $0,5 \mathrm{ccm}$ Suprareninlösung enthält $1 / 100 \mathrm{mg}$ Suprarenin.

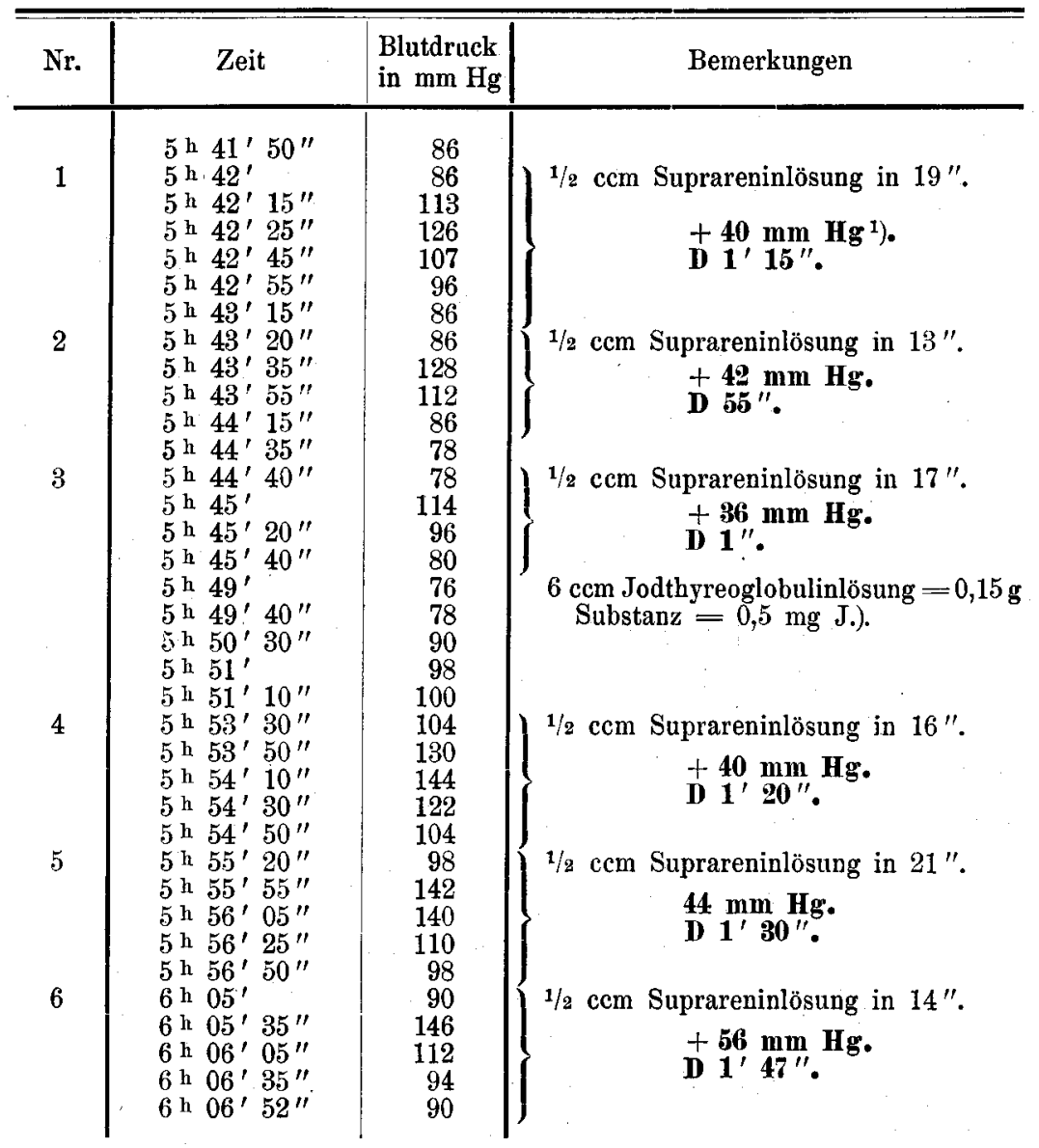

1) Das +-Zeichen bedeutet auf dieser und den folgenden Tabellen : Druckerhöhung, D bedeutet: Dauer der Druckerhöhung. Ich habe die Werte für beide auf den Tabellen besonders angeführt, um ein rasches Orientieren zu gestatten. 

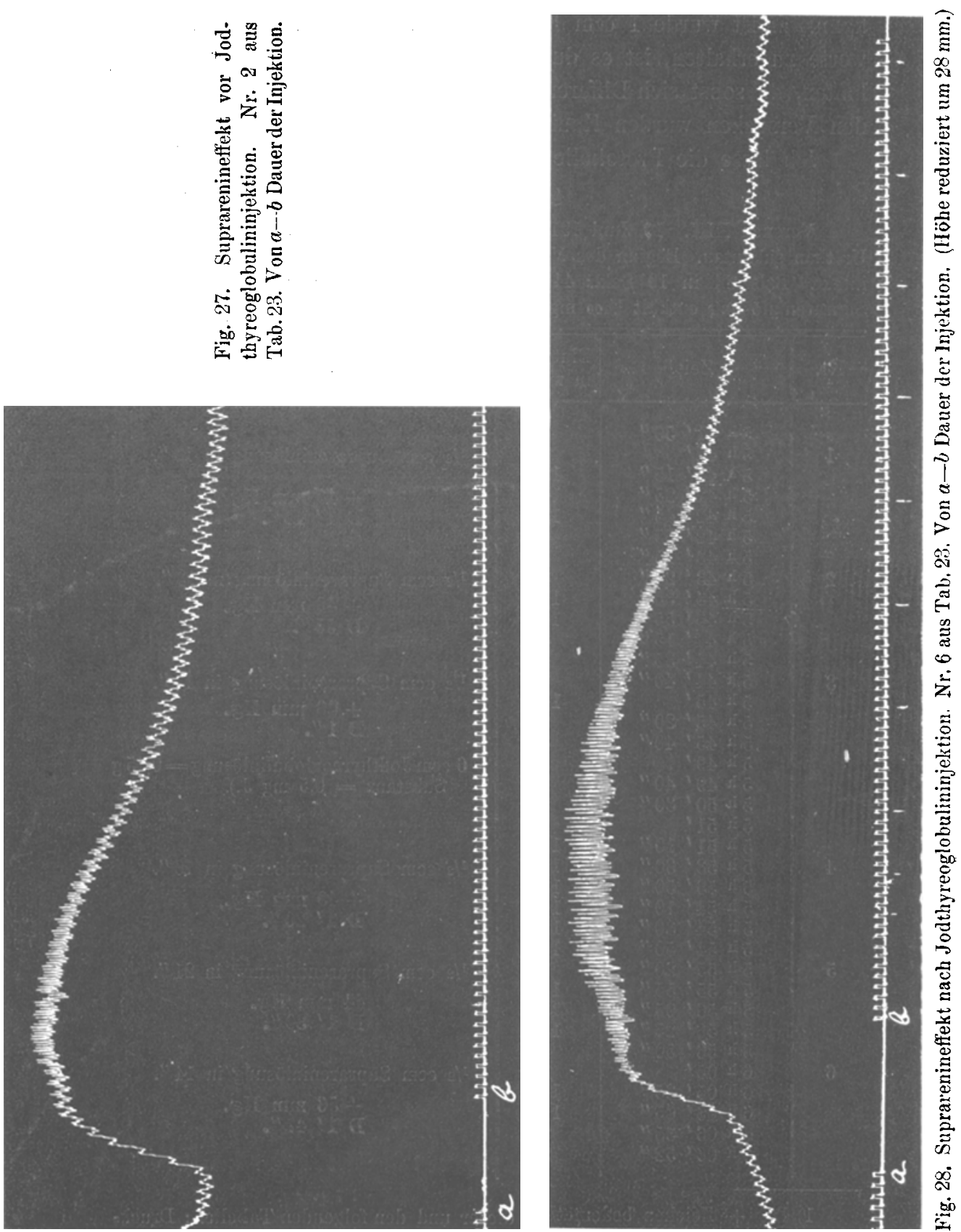
Der Versuch zeigt, dass, während die Druckzunahme unter dem Einfluss von $1 / 2 \mathrm{ccm}$ Suprareninlösung $36-42 \mathrm{~mm} \mathrm{Hg}$ betrug und die Dauer der Druckerhöhung 55 Sekunden bis 1 Minute 15 Sekunden ausmachte, erstere nach der Jodthyreoglobulininjektion 40-56 mm und letztere 1 Min. 20 Sek. bis 1 Min. 47 Sek. betrug. Jede folgende Suprareninzufuhr löst einen stärkeren Effekt aus, was sowohl die Höhe als die Dauer der Druckerhöhung anbetrifft, die Wirksamkeit des Jodthyreoglobulins ist also in ständigem Wachsen begriffen. Das ist 15 Minuten nach Schluss der Injektion noch der Fall.

Die Fig. 27 und 28 geben die Wirkung vor und nach der Jorthyreoglobulininjektion wieder.

\section{Tabelle 24 .}

Versuch XIV. 23. Juni 1913. Kaninchen, Gewicht 2500 g. 4 Uhr 2 g Urethan subkutan. Beginn des Versuches 5 Uhr 15 Min. Vagi und Depressoren intakt. $0,5 \mathrm{ccm}$ Suprareninlösung $=1 / 100 \mathrm{mg}$ Suprarenin.

\begin{tabular}{|c|c|c|c|}
\hline $\mathrm{Nr}$. & Zeit & $\begin{array}{l}\text { Blutdruck } \\
\text { in } \mathrm{mm} \mathrm{Hg}\end{array}$ & Bemerkungen \\
\hline 5 & 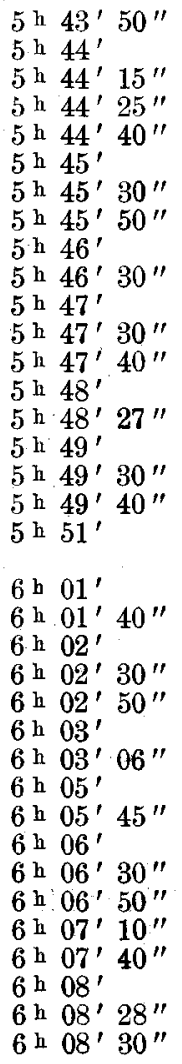 & $\begin{array}{r}90 \\
90 \\
132 \\
155 \\
130 \\
106 \\
96 \\
96 \\
98 \\
160 \\
116 \\
102 \\
94 \\
90 \\
166 \\
116 \\
100 \\
98 \\
98 \\
\\
82 \\
156 \\
126 \\
102 \\
89 \\
88 \\
80 \\
78 \\
157 \\
136 \\
110 \\
100 \\
166 \\
142 \\
118 \\
100 \\
98\end{array}$ & 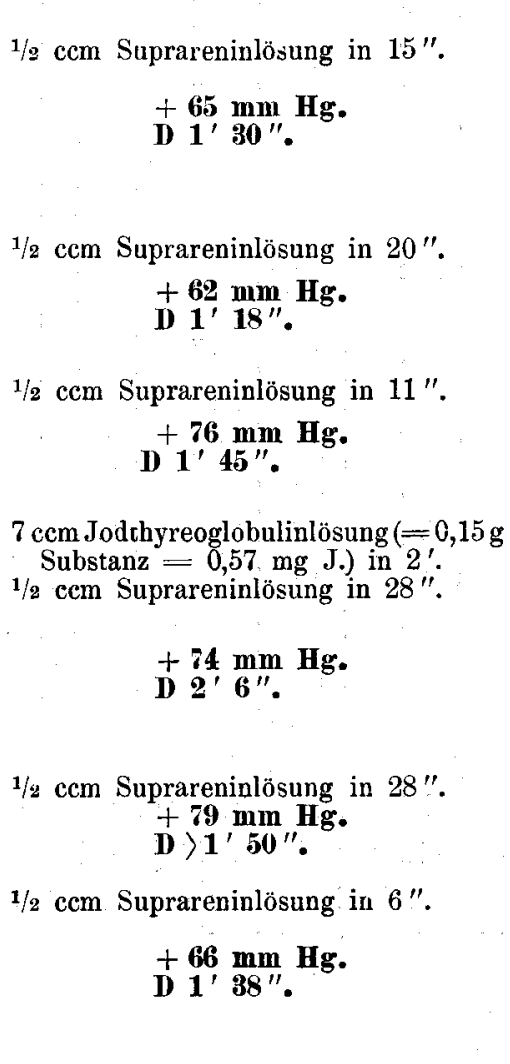 \\
\hline
\end{tabular}


Während hier die Druckzunahme vor der Jodthyreoglobulin. zufuhr $62-76 \mathrm{~mm} \mathrm{Hg}$ und die Dauer der Druckerhöhung $1 \mathrm{Min}$. 30 Sek. bis 1 Min. 45 Sek. betrug, machte nach derselben erstere $66-79 \mathrm{~mm} \mathrm{Hg}$ und letztere 1 Min. 38 Sek. bis 2 Min. 6 Sek. aus. Hier war die Wirkung 8 Min. nach Schluss der Jodthyreoglobulininjektion am stärksten. Allmählich wird die Wirkung wieder schwächer, wie das bei wiederholter Adrenalinzufuhr eine bekannte Erscheinung ist.

Tabelle 25 .

Versuch XVI. 3. Juli 1913. Kaninchen; Gewicht 2150 g. $4^{3 / 4}$ Uhr 2 g Urethan subkutan. Beginn des Versuches $6 \mathrm{Uhr}$. Vagi intakt. 0,5 ccm Suprarenin. lösung $=1 / 100 \mathrm{mg}$ Substanz.

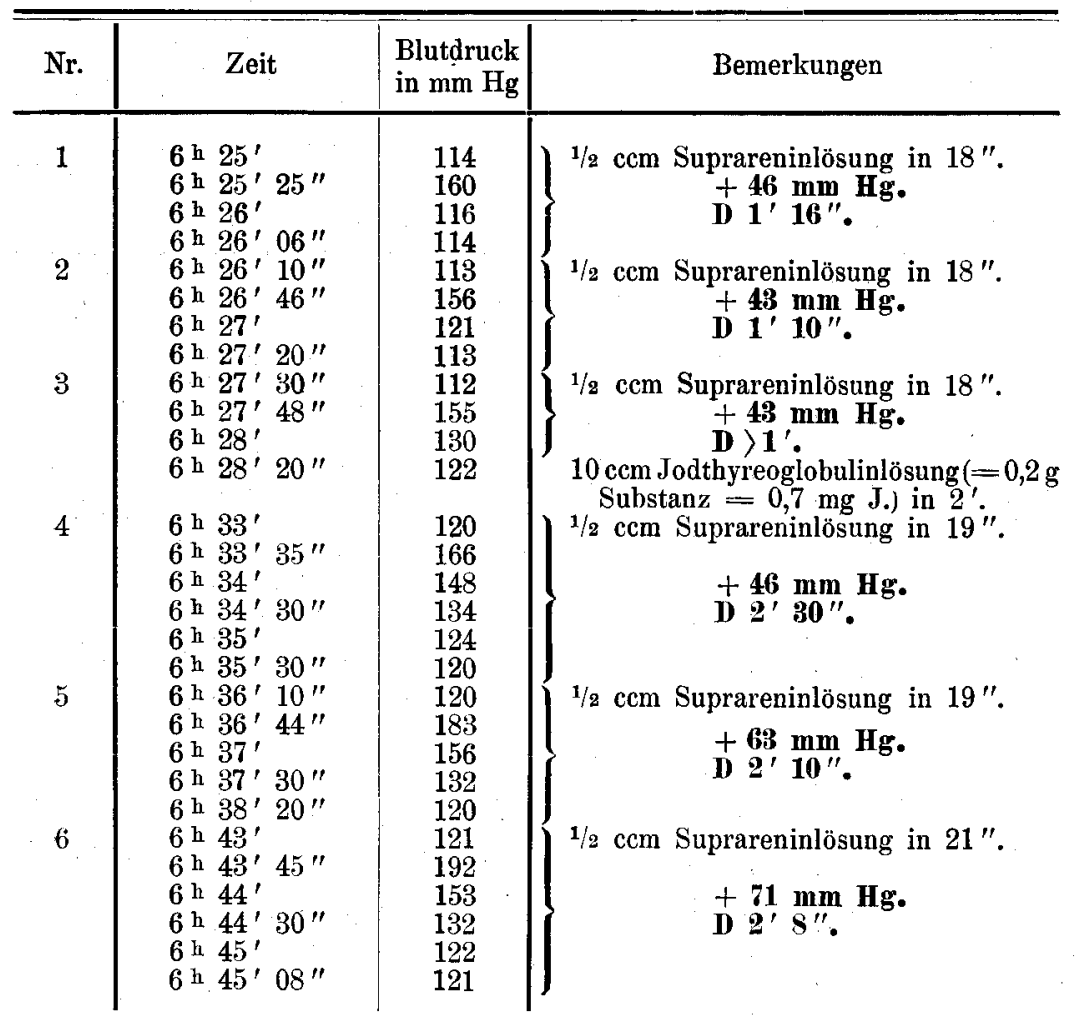

Die Druckerhöhung, welche hier vor der Jodthyreoglobulinzufuhr $43-46 \mathrm{~mm} \mathrm{Hg}$ betragen hatte, stieg nach derselben auf $63-71$, während die Dauer des Effektes von 1 Min. 6 Sek. auf 2 Min. 8 Sek. bis 2 Min. 30 Sek. heranwuchs. Die Wirkung war noch ungemindert vorhanden 14 Minuten nach Schluss der Injektion. Anfangs war sie 
nur in bezug auf die Daner des Effektes vorhanden. Die stärkste Wirkung auf die Höhe der Druckvermehrung war erst 12 Min. nach Schluss der Injektion bemerkbar.

Siehe bez. dieses Versuches die Fig. 29 und 30.
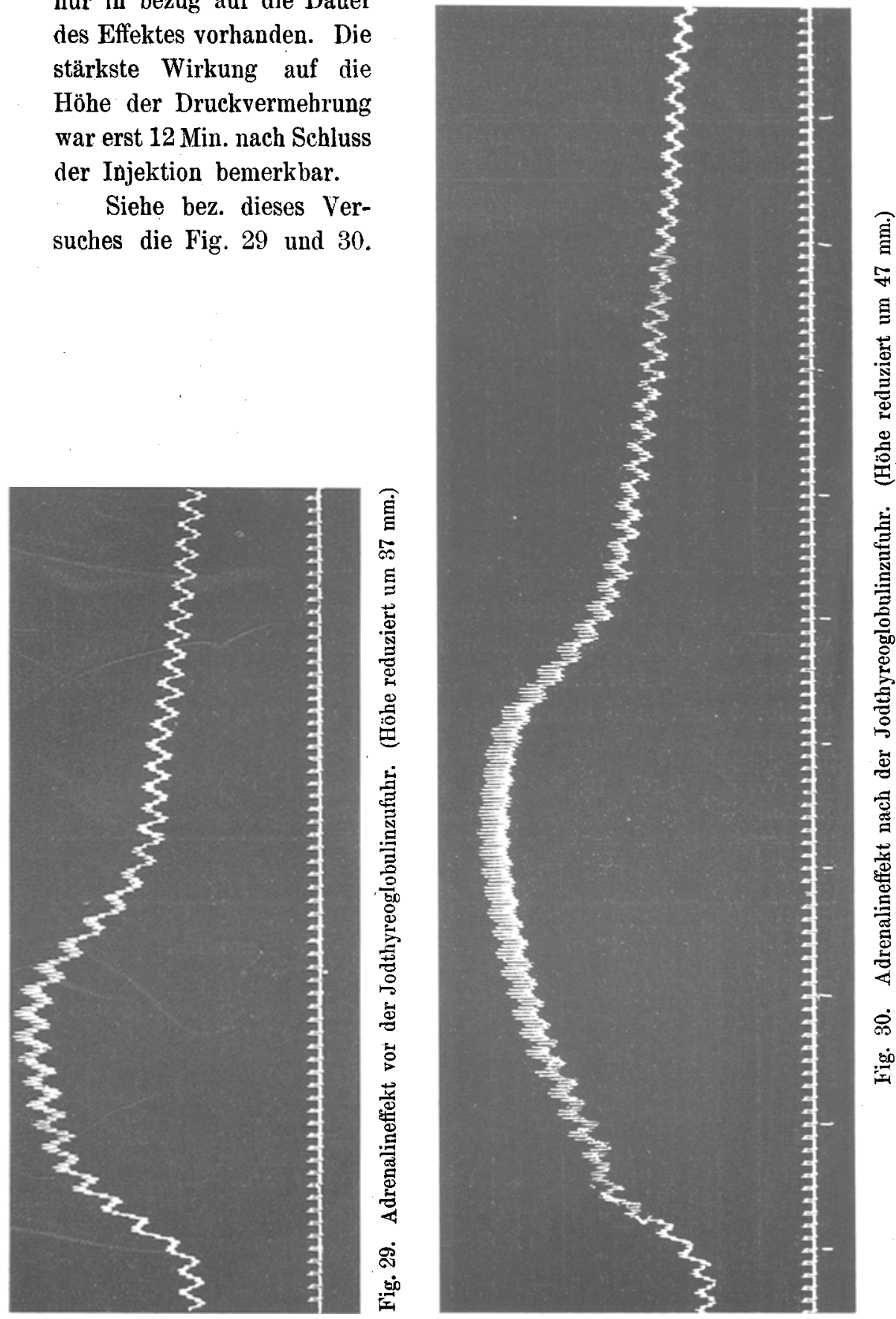
Tabelle 26.

Versuch XLV. 22. Mai 1914. Katze, Gewicht 2450 g. 4 Uhr 15 Min. $2^{1 / 2} \mathrm{ccm}$ Paraldehyd. Äthernarkose. Vagi, Depressoren, Sympathici beiderseits durchschnitten. $1 \mathrm{ccm}$ Suprareninlösung $=1 / 10 \mathrm{mg}$ Suprarenin.

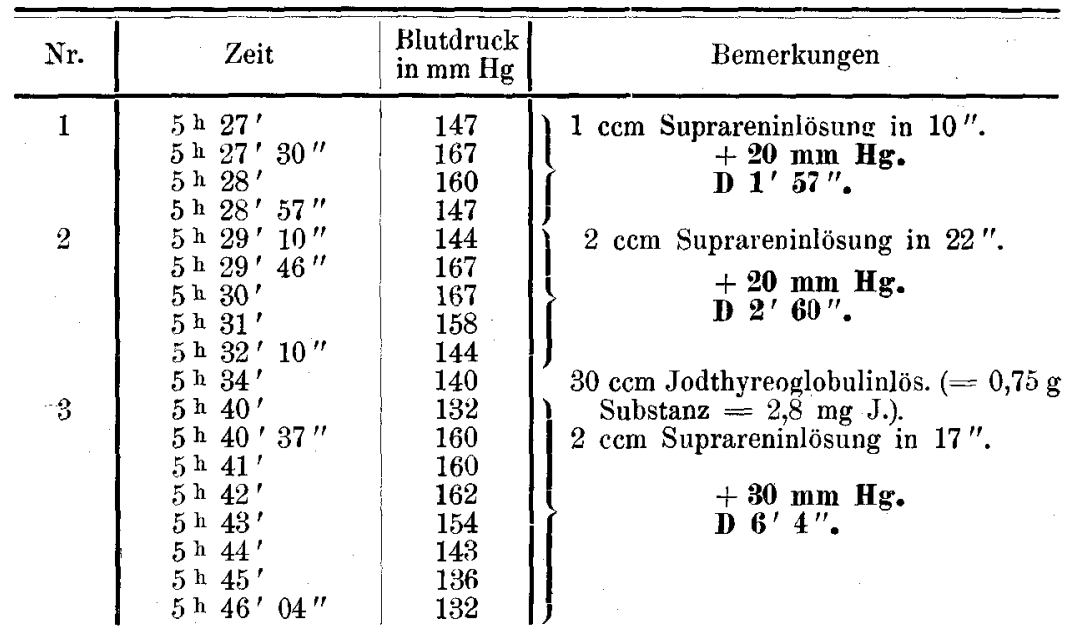

Auch hier, bei der Katze, wird die Suprareninwirkung bedeutend verstärkt, was wenigstens die Dauer des Effektes anbelangt. Während sie vor der Injektion 2 Min. 60 Sek. betrug, stieg sie nach derselben auf 6 Min. 4 Sek. Die Druckerböhung nahm um $10 \mathrm{~mm} \mathrm{zu.}$

Tabelle 27.

Versuch XLIV. 13. Mai 1914. Hund, Gewicht $20 \mathrm{~kg}$. Vagi und Depressoren intakt. $1 \mathrm{ccm}$ Suprareninlösung $=0,07 \mathrm{mg}$ Suprarenin.

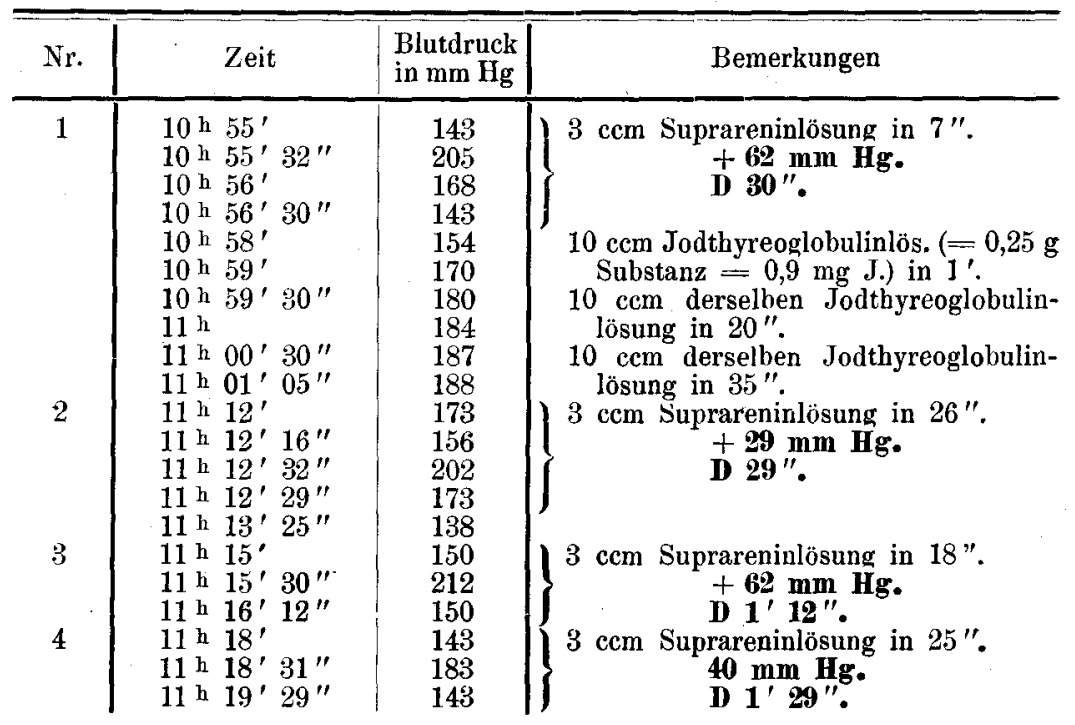


Auch beim Hund ist somit eine Verstärkung der Jodthyreoglobulinwirkung zu beobachten, die sich in diesem Versuch zwar nur hinsichtlich der Dauer der Wirkung bemerkbar gemacht hat. Während der Druck vor der Injektion nach 30 Sek. wieder auf die vorherige Höhe sank, brauchte er zu diesem Ende nach derselben 1 Min. 29 Sek. Die Wirkung nahm 14 Min. nach der Injektion noch zu und war 18 Min. danach noch gleich stark.

Fassen wir das Resultat aller dieser Versuche zusammen, so ersehen wir also, dass das Jodtby reoglobulin die Eigenschaft hat, die Wirkung des Adrenalins auf den Blutdruck, was sowohl die Intensität als die Dauer derselben anbelangt, bedeutend zu verstärken.

Es erübrigt zu erwähnen, dass nach Adrenalin-(Suprarenin-) Zufuhr die intravenöse Irjektion von Jodthyreoglobulin sozusagen regelmässig eine vorübergehende mehr oder weniger ausgesprochene Blutdrucksenkung, gefolgt oder auch nicht von einer vorübergehenden Blutdruckerhöhung, herbeiführt. Wir haben gesehen, dass beim nicht vorbehandelten Tier Jodthyreoglobulin keine Blutdruckveränderung bewirkt. Nach der vasokonstriktorischen Wirkung des Adrenalins kommt somit dem Jodthyreoglobulin eine solche zu. In einer späteren Abhandlung werde ich zeigen, dass auch nach der Zufuhr anderer den Blutdruck beeinflussenden Substanzen das Jodthyreoglobulin depressorische Eigenschaften besitzt. Dabei ist zu beachten, dass diese Wirkung eintritt, nachdem der Effekt der den Blutdruck beeinflussenden Substanz vollständig abgeklungen ist. Das Jodthyreoglobulin hat also die Eigenschaft, eine latent gewordene (abgeklungene) Reizwirkung wieder wachzurufen. Es macht die Antezedenzien wieder manifest.

Kraus und Friedenthal ${ }^{1}$ ) geben an, dass auf der Höhe des Adrenalineffektes („während der maximalen Wirkung") die elektrische Erregbarkeit des Vagus herabgesetzt bzw. aufgehoben sei. Diese Ansicht ist irrtümlich. Schon 01 iver und $\mathrm{S}$ chäfer ${ }^{2}$ ) haben gezeigt, dass der Vagus durch Adrenalin miterregt wird, indem sie dartaten, dass der Vorhof während des Adrenalineffektes in Diastole stehen bleibt und der Ventrikel seltener schlägt, während beides ausbleibt, wenn beide Vagi durchschnitten oder durch Atropin gelähmt sind. Ich habe Kraus' und Friedentbal's Angabe nachgeprüft und bin nicht imstande, sie zu bestätigen. Ich habe den Vagus sowohl

1) loc. cit.

2) loc. cit. 
im aufsteigenden wie im absteigenden Ast der Kurve faradisch erregbar gefunden, wie folgende Versuche lehren.

$$
\text { Tabelle } 28 .
$$

Versuch XXII. 31. Oktober 1913. Kaninchen, Gewicht 2225 g. $3 \mathrm{Uhr}$ 30 Min. $1,5 \mathrm{~g}$ Urethan subkutan. Vagi nnd Depressoren intakt. $0,5 \mathrm{ccm}$ Adrenalinlösung $=1 / 100 \mathrm{mg}$ Substanz.

\begin{tabular}{|c|c|c|c|c|c|c|c|c|}
\hline \multirow{2}{*}{ Nr. } & \multirow{2}{*}{ Zeit } & \multirow{2}{*}{ 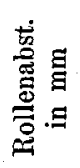 } & \multirow{2}{*}{ 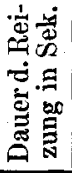 } & \multicolumn{2}{|c|}{$\begin{array}{c}\text { Pulszahl in } 10^{\prime \prime} \\
\text { bei Reizung }\end{array}$} & \multirow{2}{*}{ 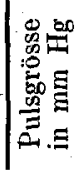 } & \multirow{2}{*}{ 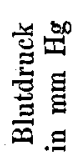 } & \multirow{2}{*}{ Bemerkungen } \\
\hline & & & & $\begin{array}{c}\text { des l. } \\
\text { Vagus }\end{array}$ & des $r$. & & & \\
\hline \multirow{9}{*}{$\begin{array}{l}1 \\
2 \\
3\end{array}$} & $6 \mathrm{~h} 10^{\prime} 55^{\prime \prime}$ & & & - & - & 一 & 95 & \multirow{9}{*}{$\begin{array}{l}1 / 2 \text { ccm Adrenalin- } \\
\text { lösung in 5". }\end{array}$} \\
\hline & 6 h $11^{\prime}$ & 190 & 10 & 15 & - & 18 & - & \\
\hline & 6 h $14^{\prime} 50^{\prime \prime}$ & & & - & - & & 90 & \\
\hline & $6 \mathrm{~h} 15^{\prime}$ & 185 & 10 & - & 8 & 19 & - & \\
\hline & $6^{\text {h }} 16^{\prime}$ & - & - & - & - & - & 101 & \\
\hline & 6 h $16^{\prime} 10^{\prime \prime}$ & - & - & - & - & - & 124 & \\
\hline & 6 h $16^{\prime} 20^{\prime \prime}$ & - & - & - & - & - & 141 & \\
\hline & $6^{\text {h }} 16^{\prime} 40^{\prime \prime}$ & - & - & - & - & - & 121 & \\
\hline & 6 h $17^{\prime}$ & - & - & - & - & - & 101 & \\
\hline \multirow[t]{2}{*}{4} & 6 h $20^{\prime}$ & - & - & - & - & - & 88 & \multirow{6}{*}{$\begin{array}{l}1 / 2 \text { ccm Adrenalin- } \\
\text { lösung in } 5 " .\end{array}$} \\
\hline & 6 h $20^{\prime} 19^{\prime \prime}$ & & & - & - & - & 150 & \\
\hline \multirow[t]{4}{*}{5} & 6 h $20^{\prime} 26^{\prime \prime}$ & 190 & 10 & 14 & - & 18 & - & \\
\hline & 6 h $20^{\prime} 40^{\prime \prime}$ & 一 & 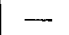 & - & - & - & 106 & \\
\hline & 6 h $20^{\prime} 50^{\prime \prime}$ & - & & - & 一 & - & 94 & \\
\hline & 6 h $21^{\prime}$ & - & - & - & - & - & 88 & \\
\hline 6 & $6^{\text {h } 22}$ & - & - & - & - & - & 85 & \multirow{7}{*}{$\begin{array}{l}1 / 2 \mathrm{ccm} \text { Adrenalin- } \\
\text { lösung in 5". }\end{array}$} \\
\hline & 6 h $22^{\prime} 19^{\prime \prime}$ & . & & - & 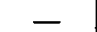 & $\sigma$ & 151 & \\
\hline \multirow[t]{5}{*}{7} & 6 与 $22^{\prime} 28^{\prime \prime}$ & 185 & 10 & - & 9 & 18 & - & \\
\hline & 6 h $22^{\prime} 42^{\prime \prime}$ & - & & - & 一 & - & 138 & \\
\hline & 6 h $22^{\prime} 50^{\prime \prime}$ & - & - & - & - & - & 106 & \\
\hline & $\begin{array}{l}6 \text { h } 23^{\prime} \\
6 \text { h } 233^{\prime} 15^{\prime \prime}\end{array}$ & - & - & $\ldots$ & $\approx$ & - & $\begin{array}{l}88 \\
85\end{array}$ & \\
\hline & & & & & & & & \\
\hline
\end{tabular}

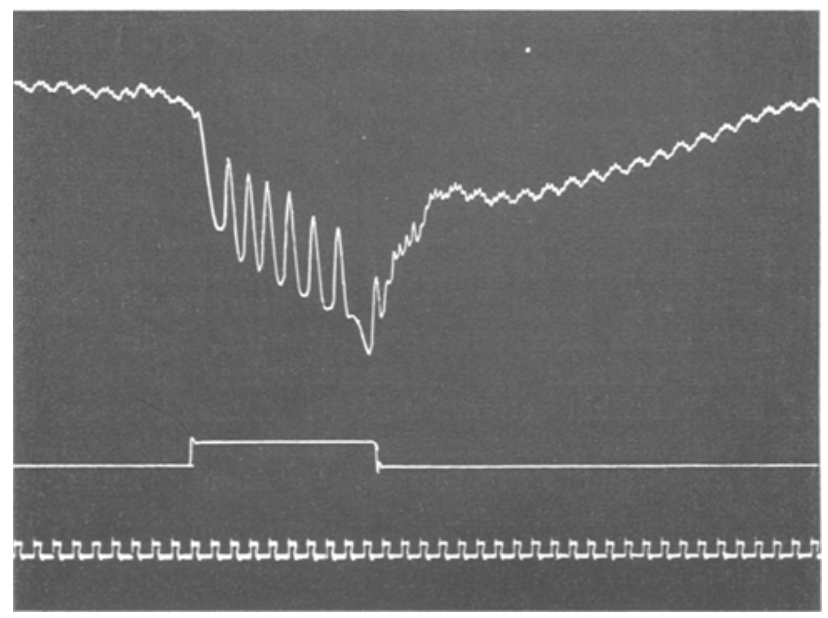

Fig. 31. Vagusreizung. Nr. 2 aus Tab. 28. 
Die Reizung mit dem faradischen Strom gibt bei gleicher Reizstärke den gleichen Ausschlag vor und während des Adrenalineffektes (absteigender Schenkel). Vgl. Fig. 31 und 32.

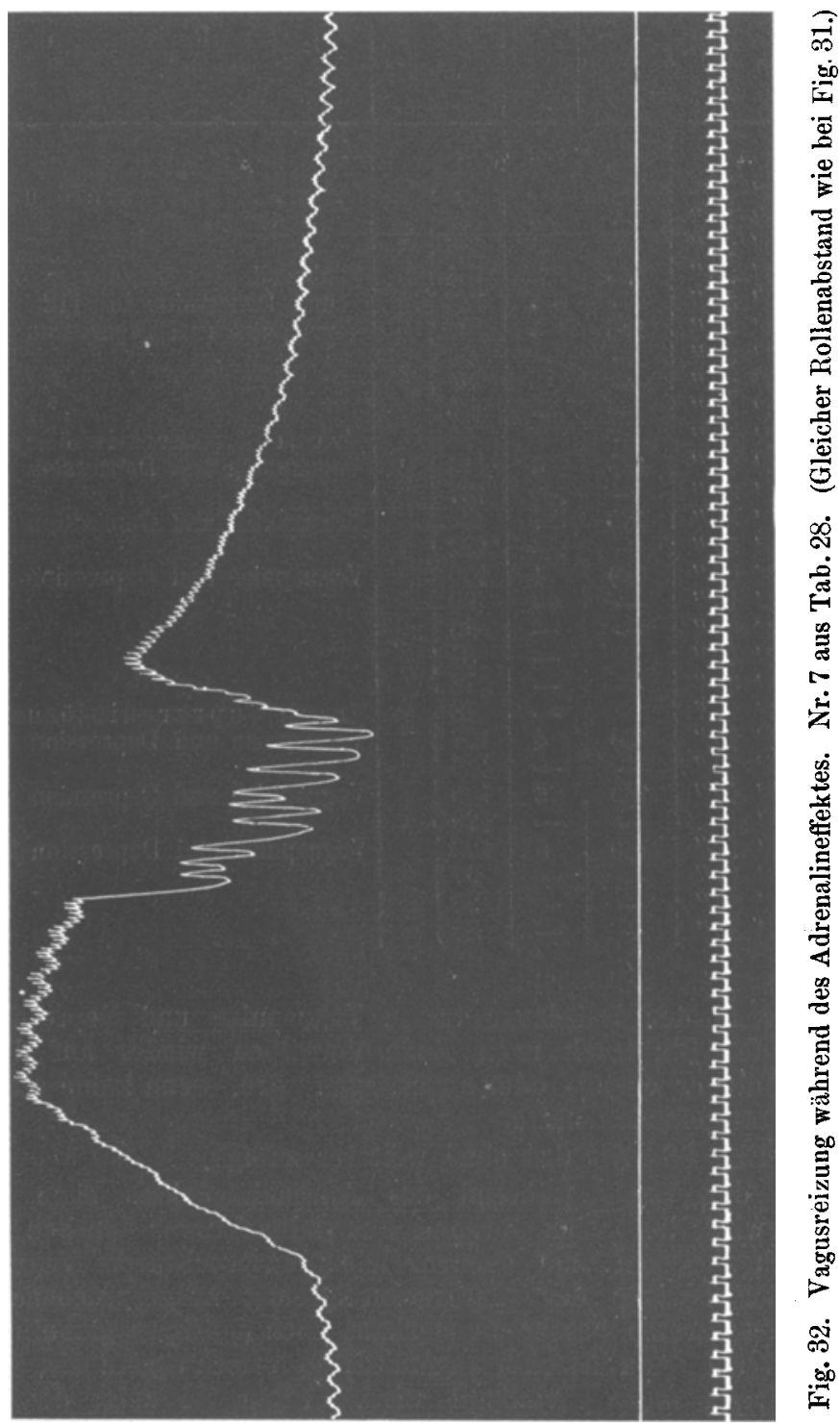


Tabelle 29.

Versuch XXIV. 6. November 1913. Ka n inchen, Gewicht $2550 \mathrm{~g}$. 41/2 Uhr $1,5 \mathrm{~g}$ Urethan. $0,5 \mathrm{ccm}$ Suprareninlösung $=1 / 100 \mathrm{mg}$ Substanz.

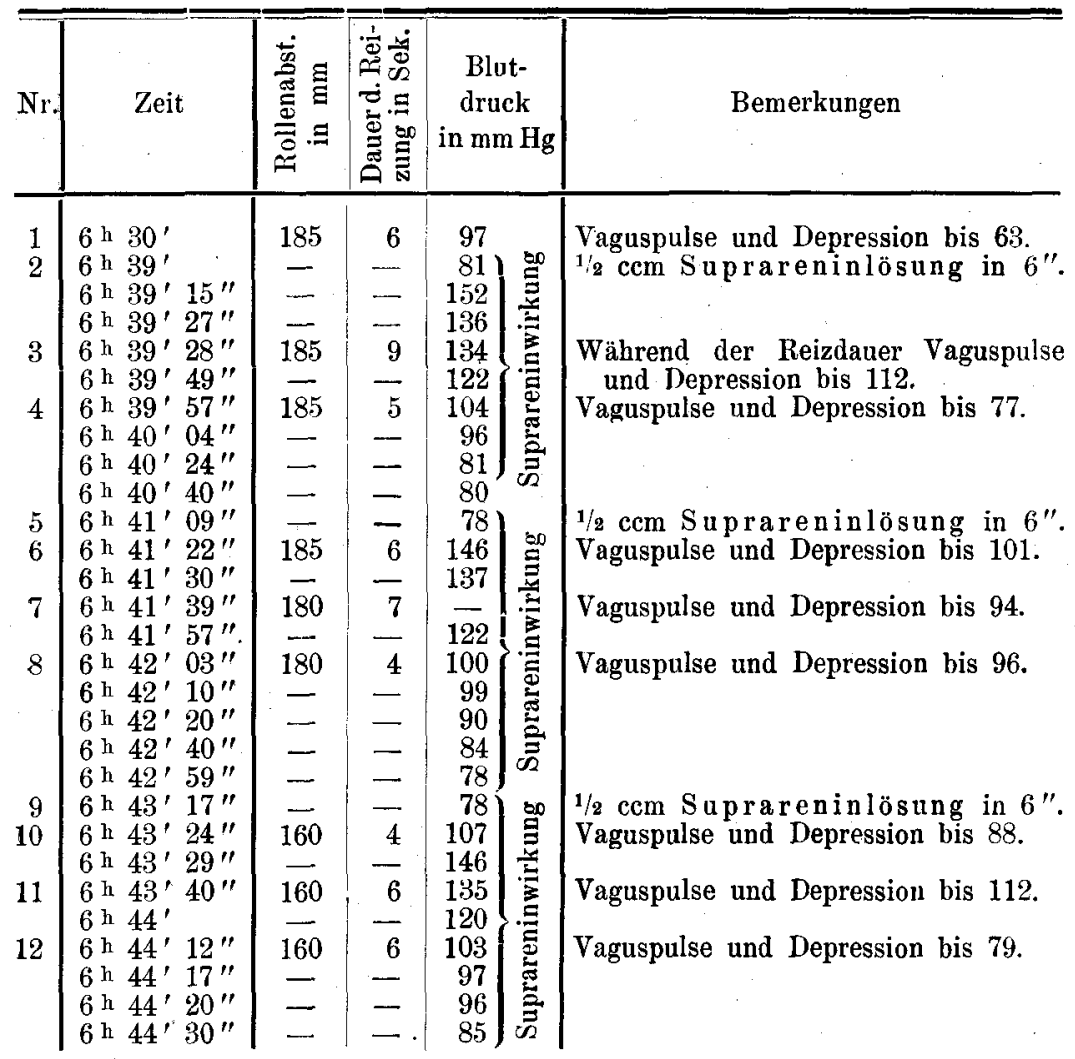

Während der Reizdauer traten Vaguspulse und Depression auf, während der Blutdruck nach Ende derselben wieder auf die Höhe ging, auf die er empor- oder abgestiegen wäre, wenn keine elektrische Reizung stattgefunden hätte.

Aus Fig. 33 tritt das ohne weiteres zutage.

Dass der Vagus während des Adrenalineffektes ohne weiteres miterregt wird, lässt sich übrigens aus vielen Kurven deutlich ersehen. Bei manchen Tieren, mit offenbar leicht ansprechbarem Vagus findet man in der Periode der Blutdrucksteigerung ausgesprochene Vaguspulse. Diese treten besonders markant nach Jodthyreoglobulineingabe hervor. Wird dann Atropin zugeführt, so verschwinden sie, während der Blutdruck nicht beeinflusst wird. Figur 34 zeigt dieses Verhalten. Bei $b$ wurden $2 \mathrm{mg}$ Atropin intravenös beigebracht.

Mehr beiläufig soll erwähnt werden, dass ich gemäss den Angaben früherer Autoren (0liver und Schäfer, Verworn, von den 
Velden usw.) während des Adrenalineffektes die Reizung des Depressors wirkungslos fand. Beim Kaninchen war eine 10 Sekunden

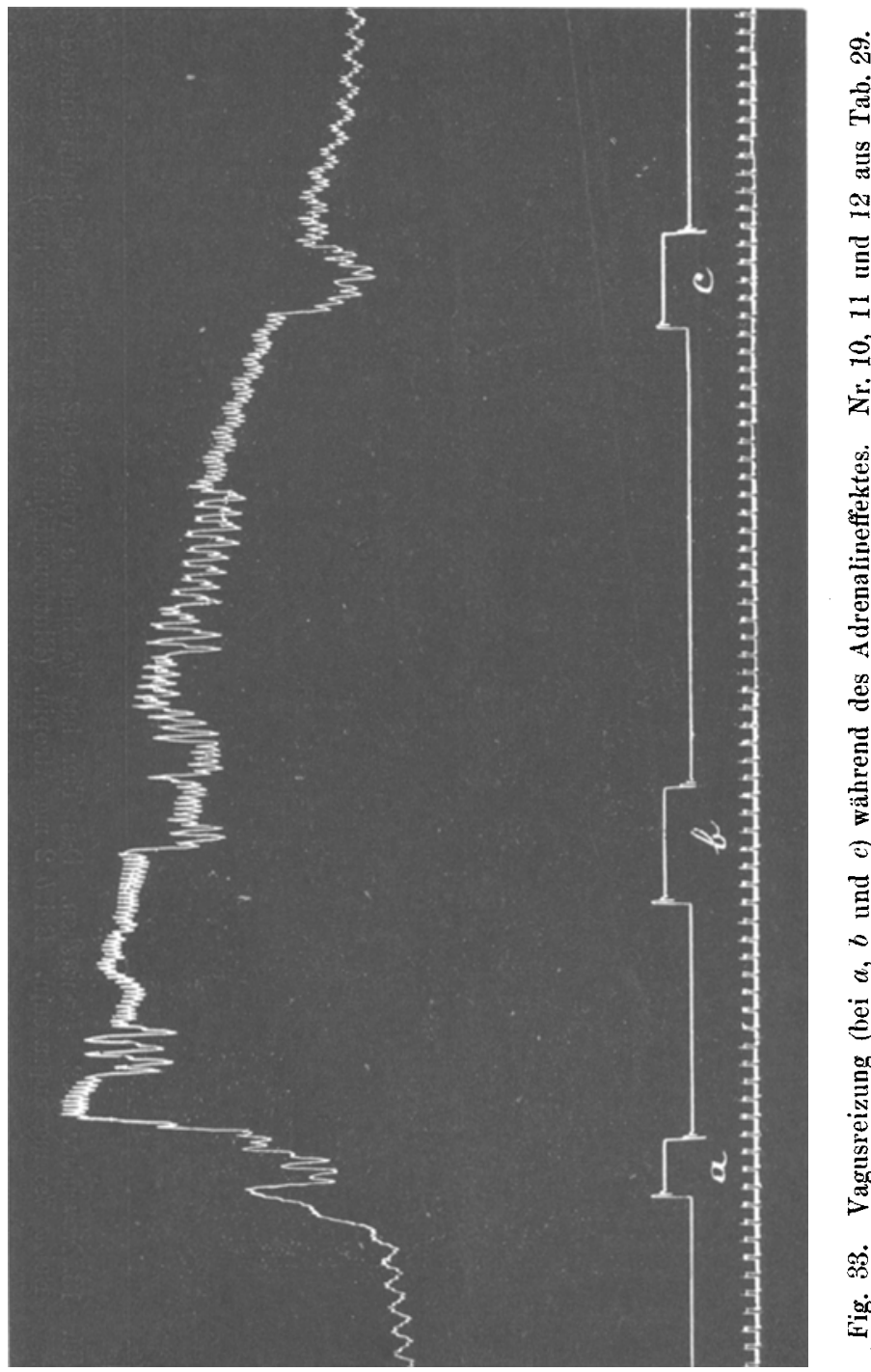

lange Reizung mit dẹm faradischen Strom bei $50 \mathrm{~mm}$ Rollenabstand effektlos, während vor und nach dem durch $1 / 100 \mathrm{mg}$ Adrenalin bewirkten Gefässkrampf die gleiche Reizung eine Depression von 40 bis $50 \mathrm{~mm}$ Quecksilber auslöste. 
556

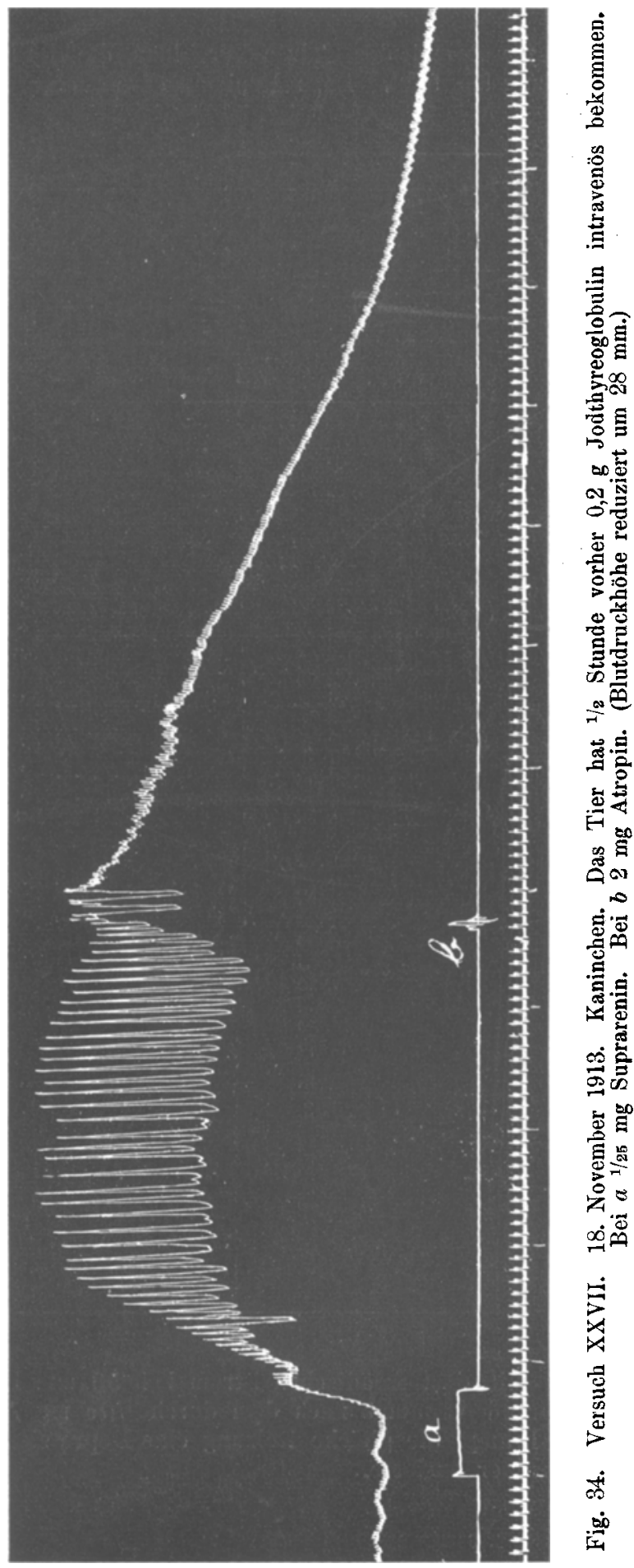




\section{Versuche mit Alkalijodthyreoglobulinat.}

Die sämtlichen im vorstehenden geschilderten Wirkungev des Jodthyreoglobulins bleiben diesem erhalten, wenn es in die Alkaliverbindung verwandelt und in diesem Zustande auf kürzere Zeit der Siedehitze ausgesetzt wird. Dieses Vorgehen hat den Vorteil, dass man es in steriler Form einem Versuchstier einverleiben kann, was eine chronische intravenöse Zufuhr mit sicherer Vermeidung septischer Erscheinungen gestattet. Bekanntlich sind bei Anlass früherer Versuche dieser Art in vielen Fällen klinische Symptome aufgetreten - ich nenne bioss Temperatursteigerung von welchen unentschieden ist, ob sie dem Schilddrüsensekret oder einer Infektion (infolge wiederholter intravenöser Injektion nicht sterilen Materials) zur Last zu legen sind. Das Vorkommen von Temperatursteigerung bei Basedow lässt es gerade als wünschenswert erscheinen, diese Frage zu entscheiden. Der Besitz eines sterilisierbaren Materials gestattet es, eine Entscheidung nach dieser Richtung zu treffen.

Ich lasse nachstehendes Versuchsprotokoll folgen, welches die gleiche Wirksamkeit auf die Ansprechbarkeit des Herzvagus und die Adrenalinempfindlichkeit beim Kaninchen für das Alkalijodthyreoglobinat dartut wie für das genuine Produkt.

Die Darstellung des Globulinats geschah auf folgende Weise: $0,5 \mathrm{~g}$ pulverisiertes, trockenes Jodthyreoglobulin wurde mit $30 \mathrm{ccm}$ Wasser angerührt, mit zirka 6 Tropfen Normal-Natronlauge versetzt und die Aufschwemmung zunächst 2 Stunden im Brutschrank belassen. Nach dieser Zeit war Lösung eingetreten, die Flüssigkeit hatte sich dunkel gefärbt. Danach wurde sie auf das Wasserbad gestellt und daselbst einige Minuten belassen, schliesslich einige Minuten lang in siedendem Wasser selbst gehalten oder auf freier Flamme einmal aufkochen gelassen. Die vollständig klare, alkalisch reagierende, dunkel gefärbte Lösung wurde alsdann mit stark verdünnter Salzsäure sorgfältig neutralisiert. Diese Prozedur brancht nicht zu ängstlich zu geschehen. Ist $\mathrm{zu}$ viel Salzsäure zugesetzt worden, was an der Ausscheidung des Jodthyreoglobulins in groben Flocken erkenntlich ist, so setzt man eine Spur Natriumbikarbonat in Substanz hinzu, und es tritt sofort Lösung ein. Die so hergerichtete Lösung ist gebrauchsbereit. 
Tabelle 30.

Versuch XXXIX. 26. Februar 1914. K an in ch en, Gewicht $2450 \mathrm{~g}$. 3 Ubr $1,6 \mathrm{~g}$ Urethan sublutan. Vagi und Depressoren intakt. 1/2 ccm Adrenalinlösung $=1 / 100 \mathrm{mg}$ Substanz.

\begin{tabular}{|c|c|c|c|c|c|c|c|}
\hline \multirow{2}{*}{ Nr. } & \multirow{2}{*}{ Zeit } & \multirow{2}{*}{ 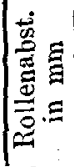 } & \multirow{2}{*}{ 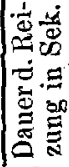 } & \multicolumn{2}{|c|}{$\begin{array}{l}\text { Pulszahl währ. } \\
\text { der Reizung }\end{array}$} & \multirow{2}{*}{ 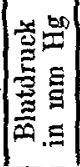 } & \multirow{2}{*}{ Bemerkungen } \\
\hline & & & & $\begin{array}{l}\text { des } 1 . \\
\text { Vagus }\end{array}$ & $\begin{array}{l}\text { des } \mathrm{r} \\
\text { Vagus }\end{array}$ & & \\
\hline \multirow{3}{*}{1} & $5^{\text {h } 35}$ & $\ldots$ & $\ldots$ & - & $\ldots$ & 77 & 1/2 ccm Adrenalinlösung in $4 "$. \\
\hline & 5 ㄴ $35^{\prime} 16^{\prime \prime}$ & - & $\sim$ & - & - & 126 & + $49 \mathrm{~mm} \mathrm{Hg}$. \\
\hline & 5 h $35^{\prime} 46^{\prime \prime}$ & - & - & - & - & 77 & D $46^{\prime \prime}$. \\
\hline \multirow[t]{4}{*}{2} & $5+36^{\prime} 10^{\prime \prime}$ & - & - & - & - & 68 & $1 / 2 \mathrm{ccm}$ Adrenalinlösung in $4 "$. \\
\hline & 5 h $36^{\prime} 23^{\prime \prime}$ & - & - & - & $\cdots$ & 122 & $+54 \mathrm{~mm} \mathrm{Hg}$. \\
\hline & $04 "$ & - & - & - & - & 69 & D $54 "$. \\
\hline & $\left\{\begin{array}{l}20^{\prime \prime} \\
40^{\prime \prime}\end{array}\right.$ & - & - & 二 & - & 68 & \\
\hline 3 & $5 \mathrm{~h} 38^{\prime} 56^{\prime \prime}$ & 180 & 10 & Depress. & - & - & \\
\hline \multirow[t]{5}{*}{4} & $30^{\prime \prime}$ & 183 & 10 & - & 10 & - & \\
\hline & 5 . $41^{\prime} 30^{\prime \prime}$ & - & - & 一 & - & 70 & $10 \mathrm{ccm}$ Alkalijodthyreoglobi- \\
\hline & 5 h $41^{\prime} 50^{\prime \prime}$ & - & $\sigma$ & 一 & - & 72 & linatlösung $\mathrm{f}=0,15 \mathrm{~g}$ sub- \\
\hline & 5 h $42^{\prime} 10^{\prime \prime}$ & - & - & - & - & 76 & stanz $=0,6 \mathrm{mg} \mathrm{J.)}$ \\
\hline & $5 \mathrm{~h}$ & - & - & 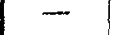 & - & 87 & Th \\
\hline \multirow[t]{3}{*}{5} & 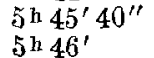 & - & 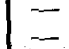 & - & - & $\begin{array}{r}92 \\
164\end{array}$ & $\begin{array}{l}\text { 1/2 } \mathrm{ccm} \text { Adrenalinlösung in } 5 " \text {. } \\
+72 \mathrm{~mm} \mathrm{Hg} \text {. }\end{array}$ \\
\hline & $\begin{array}{l}3 n 46^{\prime} \\
5 \mathrm{~s} 46^{\prime} 56^{\prime \prime}\end{array}$ & - & - & - & - & 92 & b $1,16 "$ \\
\hline & 5 h $47^{\prime} 40^{\prime \prime}$ & - & - & - & - & 78 & \\
\hline \multirow[t]{2}{*}{6} & 5 h $48^{\prime} 12^{\prime \prime}$ & - & - & 一 & - & 83 & $1 / 2 \mathrm{ccm}$ Adrenalinlösung in $6 "$. \\
\hline & $5^{\mathrm{h}} 48^{\prime} 40^{\prime \prime}$ & - & - & - & - & 170 & $+87 \mathrm{~mm} \mathrm{Hg}$ \\
\hline & $\begin{array}{l}5 \text { h } 49^{\prime} 41^{\prime \prime} \\
5 \text { h } 50^{\prime}\end{array}$ & $\overline{183}$ & $\overline{7}$ & $\sim$ & Stillstand & 83 & 101 \\
\hline 8 & 5 ․ $50^{\prime} 20^{\prime \prime}$ & 180 & 8 & Stillatand & - & - & \\
\hline 9 & $5 \mathrm{~h} 50^{\prime} 45^{\prime \prime}$ & 180 & 2 & [Stillstand] & - & 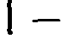 & \\
\hline
\end{tabular}

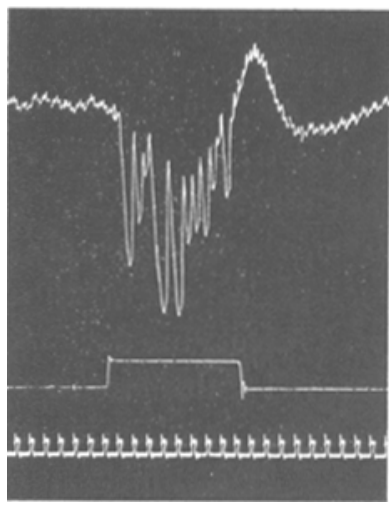

Fig. 35. Vagusreizung vor Alkalijodthyreoglobulinatzıfuhr. Nr. 4 aus Tab. 30.

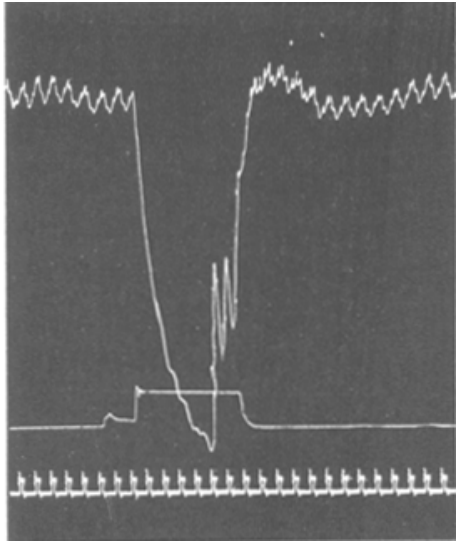

Fig. 36. Vagusreizung nach Alkalijodthyreoglobulinatzufuhr. Nr. 7 aus Tab. 30. 
Der Adrenalineffekt ist, was sowohl Dauer wie Intensität anbelangt, verstärkt, ebenso ist auch die Ansprechbarkeit des Vagus beiderseits erböht.

Fig. 35 bis 38 geben einige Ausschnitte aus der Kurve wieder.

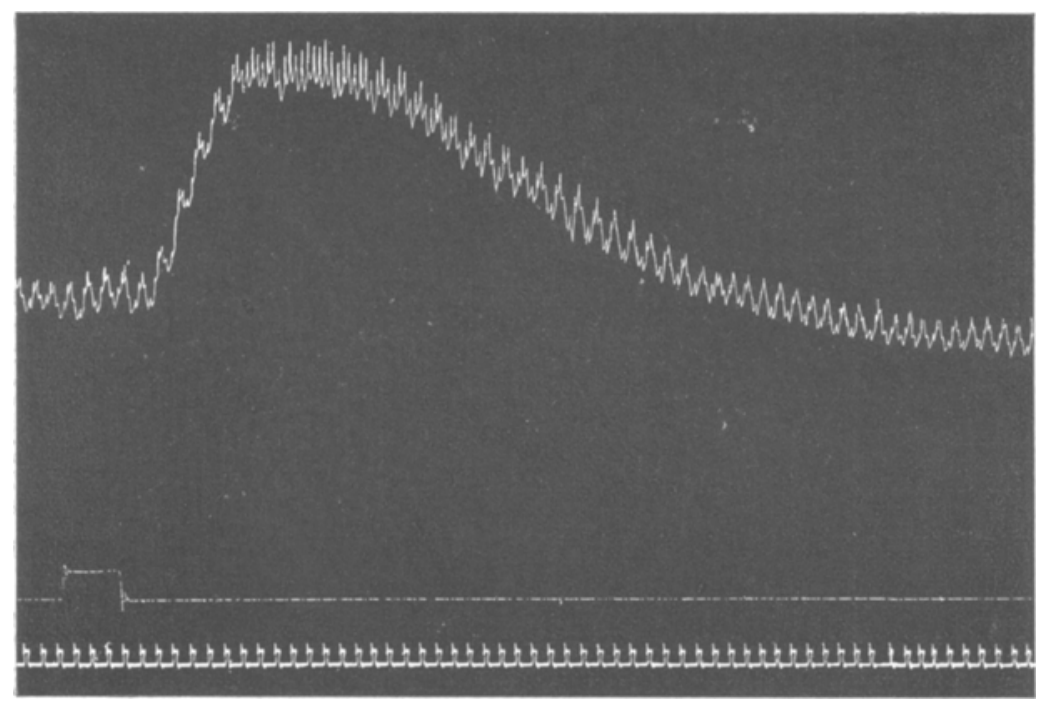

Fig. 37. Adrenalinwirkung vor Alkalijodthyreoglobulinatzufuhr. Nr. 1 aus Tab. 30.

\section{Die Bedeutung des Jodgehaltes für die Wirksamkeit des Jodthyreoglobulins.}

Nachdem eine Wirksamkeit des Jodthyreoglobulins im Sinne einer Förderung der Erregbarkeit der Herzvagusendigungen und des Depressors für den faradischen Strom und des Sympathicus bzw. dessen Ansprechorgane für deren physiologisches Reizmittel, das Adrenalin, ermittelt war, stellte sich die Frage, ob der Jodgehalt des Schilddrüsensekretes für das Zustandekommen von dessen Wirksamkeit wesentlich in Frage komme und, wenn ja, welchen Anteil das Jod daran hat.

Wie wir wissen, variiert der prozentuale Jodgehalt der Thyreoglobulinpräparate innerhalb gewisser Gŕenzen: Er hängt von verschiedenen Faktoren ab, so vom Jodgehalt der Nahrung und von der anatomischen Beschaffenheit der Drüse. Relativ jodarme Präparate lassen sich aus kolloidreichen Drüsen erhalten, insbesondere aus 





Kröpfen, Wie ich früher festgestellt habe ${ }^{1}$ ), nimmt der prozentuale Jodgehalt der aus Kolloidstrumen gewonnenen Präparate im allgemeinen mit steigendem Kolloidreichtum ab. Es frug sich, ob solche jodarme Präparate die gleiche oder eine abgeschwächte Wirksamkeit haben im Vergleich zu jodreichen.

Weiterhin sollte geprüft werden, ob dem aus dem Jodthyreoglobulin abspaltbaren Baumann'schen Jodothyrin noch die gleiche Wirksamkeit innewohne oder ob sie ihm fehle, und wenn ersteres der Fall sein sollte, ob sie dem Jodgehalt parallel gehe.

Es folgen nunmehr die Versuchsprotokolle.

Als jodarme Thyreoglobulinpräparate wählte ich solche aus Kröpfen vom Schwein und vom Menschen, ferner den frischen Inbalt von Kropfeysten. Der Jodgehalt dieser Präparate betrug 0,02 bis $0,03 \%$, war also rund 20 mal geringer als der der Präparate aus gesunden Drüsen. Das Jodothyrin war mir in freundlicher Weise von den Farbenfabriken Fr. Bayer \& Co. in Elberfeld zur Verfügung gestellt worden. Es enthielt $2,4 \%$ Jod.

\section{Tabelle 31 .}

Versuch III. 23. Juli 1912. Kaninchen, Gewicht 2250 g. 8 Uhr 2 g Urethan subkutan. Vagi und Depressoren intakt.

\begin{tabular}{|c|c|c|c|c|c|}
\hline $\mathrm{Nr}$. & Zeit & 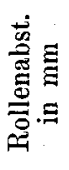 & 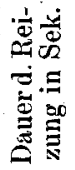 & $\begin{array}{c}\text { Pulszahl } \\
\text { während } \\
\text { d. Reizung } \\
\text { des linken } \\
\text { Vagus }\end{array}$ & Bemerkungen \\
\hline 1 & 10 h $07^{\prime}$ & 185 & 10 & keine Wirk. & \multirow{13}{*}{$\begin{array}{l}5 \mathrm{ccm} \text { Auflösung von Jodthyreoglobulin } \\
\text { aus (parenchymatösem) Kr o pf vom } \\
\text { Schwein }(=0,1 \mathrm{~g} \text { Substanz }=0,02 \mathrm{mgJ} \text {.). } \\
\text { 15 ccm derselben Lösung }(=0,06 \mathrm{mg} \mathrm{J} .) \\
15 \mathrm{ccm} \text { Auflösung von Jodthyreoglobulin } \\
\text { aus gesunden Drüs en vom Schwein } \\
\text { und Schaf gemischt }(=0,22 \mathrm{~g} \text { Sub- } \\
\text { stanz }=0,4 \mathrm{mg} \mathrm{J} .) . \\
8 \mathrm{ccm} \text { der gleichen Lôsung }(=0,4 \mathrm{mg} \mathrm{J} .)\end{array}$} \\
\hline & $10^{\text {h }} 16^{\prime}$ & & & & \\
\hline 2 & 10 h $20^{\prime}$ & 185 & 10 & 32 & \\
\hline 3 & 10 h $22^{\prime}$ & 185 & 10 & 34 & \\
\hline 4 & $10 \mathrm{~h} 22^{\prime} 10^{\prime \prime}$ & $\overline{185}$ & 10 & 22 & \\
\hline $\begin{array}{l}4 \\
5\end{array}$ & $10^{\mathrm{h}} 29^{\prime}$ & $\begin{array}{l}180 \\
185\end{array}$ & 10 & 22 & \\
\hline & $10^{\text {h } 34^{\prime}}$ & - & - & - & \\
\hline 6 & $10^{\text {h } 38^{\prime}}$ & 185 & 10 & 19 & \\
\hline 7 & $10^{\text {h }} 39^{\prime}$ & 185 & 10 & 20 & \\
\hline & $10^{\text {b }} 40^{\prime}$ & - & $\div$ & - & \\
\hline 8 & $10^{\text {b } 44^{\prime}}$ & 185 & 10 & 13 & \\
\hline $\begin{array}{r}9 \\
10\end{array}$ & 10 h $47^{\prime}$ & 185 & 10 & 15 & \\
\hline & $10 \mathrm{n} 32^{\prime}$ & $18 b$ & 10 & 17 : & \\
\hline
\end{tabular}

1) A. Oswald, Weiteres über das Thyreoglobulin. Hofmeister's Beitr. z. chem. Physiol. u. Pathol. Bd. 2 S. 545. 1902. - Vgl. auch A. Os wald, Die Chemie und Physiologie des Kropfes. Virchow's Arch. Bd. 169 S. 444. 1902. 
Nach Zufubr von Jodthyreoglobulin aus Kröpfen vom Schwein nimmt die Ansprechbarkeit des Vagus anfangs nicht, später nur wenig zu. Dagegen ist eine Zunahme zu konstatieren nach der Einverleibung von Jodthyreoglubulin aus gesunden Drüsen (vom Schwein oder Hammel).

\section{Tabelle 32.}

Versuch IV. 25. Juli 1912. Kaninchen, Gewicht 2350 g. 8 Uhr 2 g Urethan subkutan. Vagi intakt.

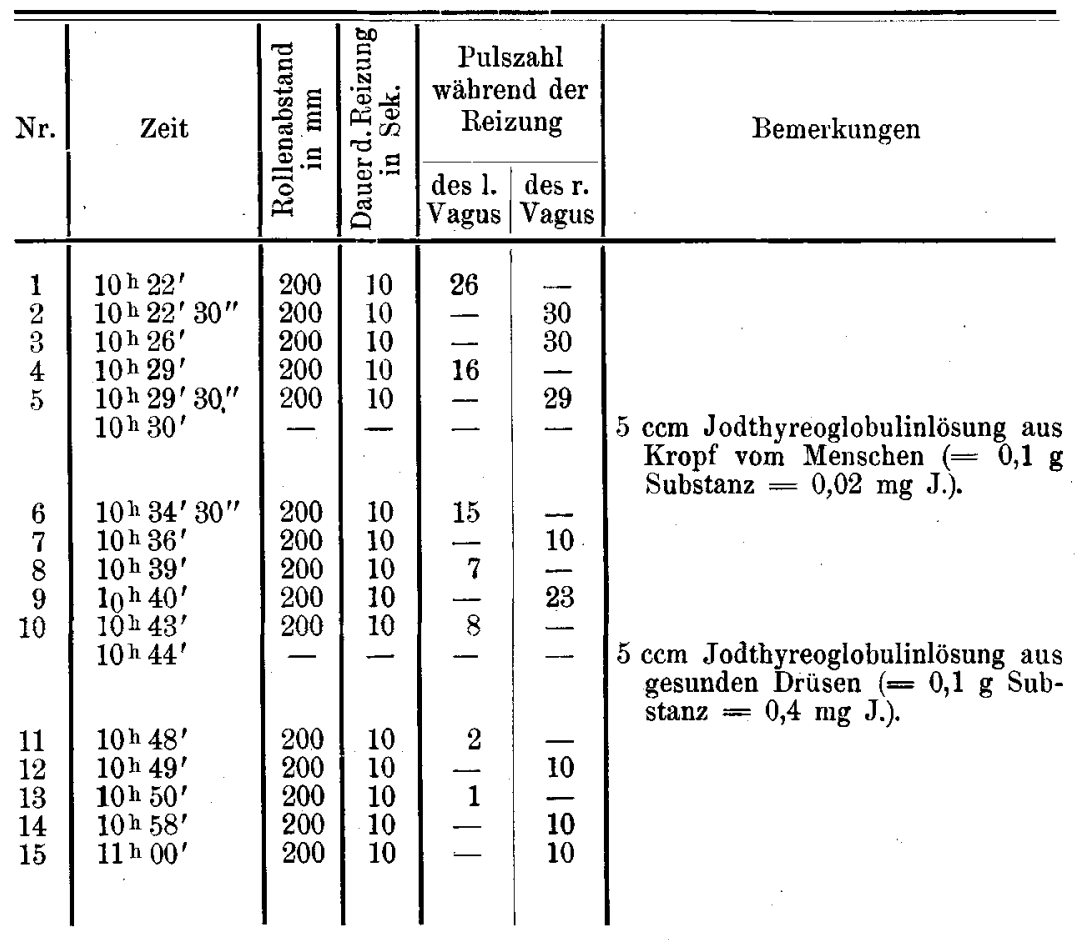

In diesem Versuch zeigte sich eine geringe Zunahme der Ansprechbarkeit sowohl des rechten wie des linken Vagus nach Zufuhr vou Jodthyreoglobulin aus Kröpfen, die nach Zufuhr von solchem aus gesunden Drüsen erheblich zunahm.

Im folgenden Versuche wurde der frische Inhalt einer K ropfcyste vom Menschen geprüft, der gelegentlich einer Operation gewonnen wurde. Der Cysteninhalt pflegt in der Regel sehr jodarm zu sein. Der Inbalt zeigte zähe Konsistenz und hellbraungelbe Farbe. Er war frei von frischem und verändertem Blutfarbstoff. 
Tabelle 33.

Versuch V. 27. Juli 1912. Kaninchen, Gewicht 2850 g. 8 Uhr 2 g Urethan subkutan. Vagi intakt.

\begin{tabular}{|c|c|c|c|c|c|}
\hline $\mathrm{Nr}$. & Zeit & $\begin{array}{l}\text { Rollen- } \\
\text { abstand } \\
\text { in mm }\end{array}$ & $\begin{array}{l}\text { Reiz- } \\
\text { dauer } \\
\text { in Sek. }\end{array}$ & $\begin{array}{c}\text { Pulszahl } \\
\text { während der } \\
\text { Reizung des } \\
\text { r. Vagus }\end{array}$ & Bemerkungen \\
\hline $\begin{array}{r}1 \\
2 \\
3 \\
4 \\
5 \\
6 \\
7 \\
8 \\
\\
9 \\
10 \\
11 \\
12 \\
13\end{array}$ & 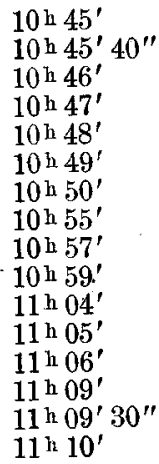 & $\begin{array}{l}200 \\
210 \\
220 \\
230 \\
210 \\
230 \\
- \\
220 \\
230 \\
- \\
220 \\
230 \\
220 \\
200 \\
210 \\
200\end{array}$ & $\begin{array}{r}8 \\
10 \\
10 \\
10 \\
10 \\
10 \\
10 \\
10 \\
10 \\
10 \\
10 \\
9 \\
10 \\
10\end{array}$ & $\begin{array}{r}4 \\
13 \\
26 \\
40 \\
9 \\
25 \\
6 \\
16 \\
16 \\
22 \\
23 \\
4 \\
17 \\
17\end{array}$ & $5 \mathrm{ccm}$ genuiner Cystengehalt. \\
\hline
\end{tabular}

Es zeigte sich, dass die Wirkung nur schwach war, indem die Pulszahl bei gleichstarkem Reiz nur unbedeutend oder gar nicht abnahm.

Das Ergebnis dieser drei Versucbe geht dahin, dass jodarme Präparate eine relativ geringere Wirksamkit entfalten als jodreichere und dass mit steigendem Jodgehalt die Wirksamkeit zunimmt.

Nun wurde Jodothyrin auf seine Wirksamkeit geprüft. Folgende Versuche geben darüber Aufsehluss. Zunächst wurde der Einfluss auf die Herzvagusendigungen gepruft.

\section{Tabelle 34 .}

Versuch VI. 22. Mai 1913. Kaninchen, Gewicht 2350 g. $3^{1 / 2}$ Uhr 2 g Urethan subkutan. Beide Vagi abgebunden und durchschnitten.

\begin{tabular}{|c|c|c|c|c|c|}
\hline Nr. & Zeit & $\begin{array}{l}\text { Rollen- } \\
\text { abstand } \\
\text { in } \mathrm{mm}\end{array}$ & $\begin{array}{l}\text { Reiz- } \\
\text { dauer } \\
\text { inSek. }\end{array}$ & $\mid \begin{array}{c}\text { Pulszahl } \\
\text { in 10Sek. bei } \\
\text { Reizung des } \\
\text { 1. Vagus }\end{array}$ & Bemerkungen \\
\hline $\begin{array}{l}1 \\
2 \\
3 \\
4 \\
5\end{array}$ & 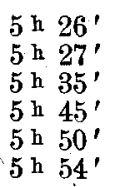 & $\begin{array}{l}195 \\
200 \\
-195 \\
195 \\
195\end{array}$ & $\begin{array}{l}10 \\
10 \\
10 \\
10 \\
10\end{array}$ & $\begin{array}{l}16 \\
16 \\
9 \\
7 \\
9\end{array}$ & $5 \mathrm{ccm}$ Jodothyrinlösung $=1,5 \mathrm{mg} \mathrm{J}$.$) .$ \\
\hline
\end{tabular}


Die Pulszahl sank bei Reizung des linken Vagus vor Jodothyrinbeibringung auf 16 , nacher auf 9 und 7. Das Jodothyrin erhöhte also die Ansprechbarkeit des Vagus.

Tabelle 35 .

Versuch VII. 27. Mai 1913. Kaninchen, Gewicht 2250 g. 3 Uhr $1,5 \mathrm{~g}$ Urethan. Vingi intakt.

\begin{tabular}{|c|c|c|c|c|c|c|}
\hline \multirow[t]{2}{*}{$\mathrm{Nr}$} & \multirow{2}{*}{ Zeit } & \multirow{2}{*}{$\begin{array}{l}\text { Rollen- } \\
\text { abstand } \\
\text { in mm }\end{array}$} & \multirow{2}{*}{$\begin{array}{l}\text { Daner } \\
\text { d. Rei- } \\
\text { zung } \\
\text { in Sek. }\end{array}$} & \multicolumn{2}{|c|}{$\begin{array}{l}\text { Pulszahl in } 10 \text { Sek. } \\
\text { bei Reizung des }\end{array}$} & \multirow{2}{*}{ Bemerkungen } \\
\hline & & & & 1. Vagus & r. Vagus & \\
\hline 1 & 5 h 27 & 200 & 10 & keine Wirk. & & \\
\hline 2 & 5 h $31^{\prime}$ & 195 & 10 & - & keine Wirk. & \\
\hline 3 & 5 h $32^{\prime}$ & 195 & 10 & 7 & & \\
\hline 4 & 5 h $34^{\prime}$ & 190 & 10 & - & keine Wirk. & \\
\hline 5 & 5 h $38^{\prime}$ & 196 & 10 & 15 & - & \\
\hline 6 & 5 h $48^{\prime}$ & 186 & 10 & - & 11 & \\
\hline 7 & 5 h $49^{\prime}$ & $\overline{100}$ & $\overline{10}$ & $\overline{10}$ & - & $6 \mathrm{ccm}$ Jodothyrinlösung \\
\hline 8 & 5 h $50^{\prime}$ & 196 & 10 & 10 & - & $(=1,8 \mathrm{mg} \mathrm{J.})$ \\
\hline 9 & 5 h $52^{\prime}$ & 186 & 10 & $\overline{1}$ & 4 & \\
\hline 10 & 5 h $55^{\prime}$ & 196 & 10 & 1 & - & \\
\hline 11 & 5 h $54^{\prime}$ & 186 & 10 & - & 8 & \\
\hline 12 & 5 h $57^{\prime}$ & 186 & 10 & - & Stillstand & \\
\hline 13 & 5 h $59^{\prime}$ & 186 & 10 & - & Stillstand & \\
\hline 14 & & 200 & 10 & 16 & - & \\
\hline 15 & 6 h 01 & 190 & 10 & - & 4 & \\
\hline 16 & $6 \mathrm{~h} 03^{\prime}$ & 200 & 10 & 9 & - & \\
\hline 17 & 6 h $04^{\prime}$ & 190 & 10 & - & 2 & \\
\hline
\end{tabular}

Auch hier sank die Pulszahl bei Reizung des linken wie des rechten Vagus mit gleich starkem Strom nach der Beibringung von Jodothyrin stärker als vorher.

\section{Tabelle 36 .}

Versuch IX. 3. Juni 1913. Kaninchen, Gewicht 2750 g. $3^{1 / 2}$ Uhr 2 g Urethan subkutan.

\begin{tabular}{|c|c|c|c|c|c|c|}
\hline \multirow[t]{2}{*}{ Nr. } & \multirow[t]{2}{*}{ Zeit } & \multirow{2}{*}{$\begin{array}{l}\text { Rollen- } \\
\text { abstand } \\
\text { in mm }\end{array}$} & \multirow{2}{*}{$\begin{array}{l}\text { Dauer } \\
\text { d. Rei- } \\
\text { zung } \\
\text { in Sek. }\end{array}$} & \multicolumn{2}{|c|}{$\begin{array}{l}\text { Pulszahl in } 10 \text { Sek. } \\
\text { bei Reizung des }\end{array}$} & \multirow[t]{2}{*}{ Bemerkungen } \\
\hline & & & & 1. Vagus & r. Vagus & \\
\hline 1 & 5 h $30^{\circ}$ & 185 & 10 & 17 & - & \\
\hline 2 & 5 h $32^{\prime}$ & 190 & 10 & 18 & - & \\
\hline 3 & 5 h $33^{\prime}$ & 190 & 10 & - & 2 & \\
\hline 4 & 5 h $34^{\prime}$ & 195 & 10 & keine Wirk. & - & \\
\hline 5 & 5 h $43^{\prime}$ & 193 & 10 & - & 11 & \\
\hline & 5 h $44^{\prime}$ & & & 11 & - & $5 \mathrm{ccm}$ Jodothyrinlösung \\
\hline 6 & 5 h $47^{\prime}$ & 185 & 10 & 11 & - & $(=1,5 \mathrm{mg} \mathrm{J})$. \\
\hline 7 & 5 h $48^{\prime}$ & 193 & 10 & - & 9 & \\
\hline 8 & 5 h $49^{\prime}$ & 185 & 10 & 9 & - & \\
\hline 9 & 5 h $50^{\prime}$ & 193 & 10 & $\overline{10}$ & 9 & \\
\hline 10 & 5 h $51^{\prime}$ & 185 & 10 & 10 & 一 & \\
\hline 11 & 5 h $52^{\prime}$ & 190 & 10 & 10 & - & \\
\hline 12 & 5 h $54^{\prime}$ & 195 & 10 & 13 & - & \\
\hline 13 & 5 h $58^{\prime}$ & 193 & 10 & - & 8 & \\
\hline 14 & 5 h $59^{\prime}$ & 185 & 10 & Stillstan 1 & - & \\
\hline
\end{tabular}


Sowohl der rechte wie der linke Vagus zeigt nach der Jodothyrininjektion eine wenn auch relativ geringe Erhöhung der Ansprechbarkeit auf den faradischen Reiz.

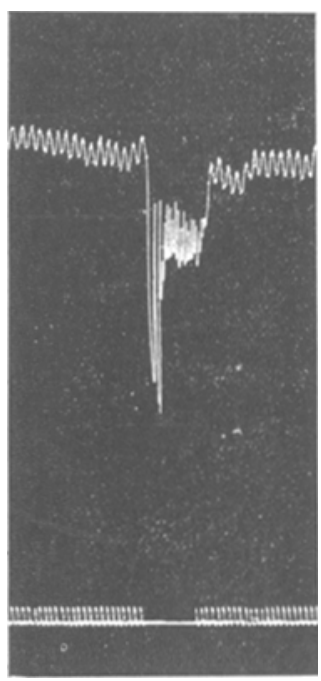

Fig. 39. Vagusreizung vor Jodothyrinzufuhr. Nr. 5 aus Tab. 37.

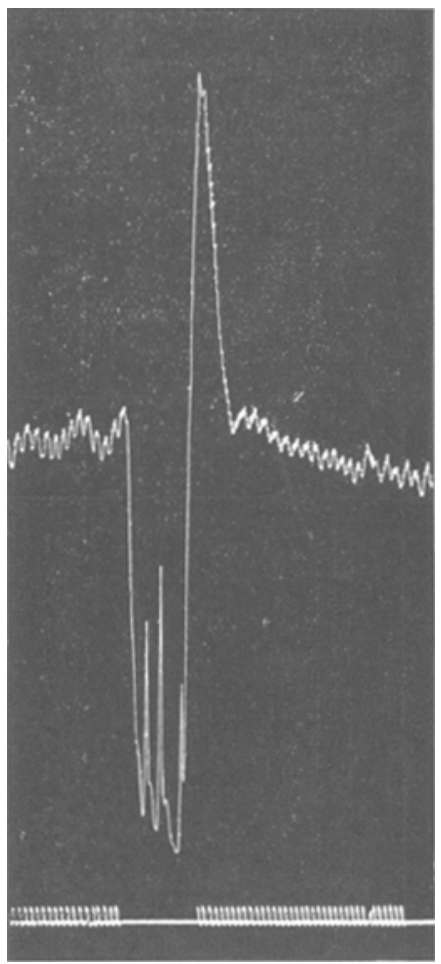

Fig. 40. Vagusreizung nach Jodothyrinzufubr. Nr. 10 aus Tab. 37.

Tabelle 37.

Versuch X. 5. Juni 1913. Kaninchen, Gewicht 2780 g. 3 Uhr 2 g Urethan subkutan. Vagi und Depressoren intakt.

\begin{tabular}{|c|c|c|c|c|c|c|}
\hline \multirow[t]{2}{*}{$\mathrm{Nr}$} & \multirow[t]{2}{*}{ Zeit } & \multirow{2}{*}{$\begin{array}{l}\text { Rollen- } \\
\text { abstand } \\
\text { in } \mathrm{mm}\end{array}$} & \multirow{2}{*}{$\begin{array}{l}\text { Dauer } \\
\text { d. Rei- } \\
\text { zung } \\
\text { in Sek. }\end{array}$} & \multicolumn{2}{|c|}{$\begin{array}{c}\text { Pulszahl in } 10 \text { Sek. } \\
\text { bei Reizung des }\end{array}$} & \multirow{2}{*}{ Bemerkungen } \\
\hline & & & & 1. Vagus & r. Vagus & \\
\hline $\begin{array}{r}1 \\
2 \\
3 \\
4 \\
5 \\
6 \\
7 \\
\\
8 \\
9 \\
10 \\
11 \\
12 \\
13\end{array}$ & 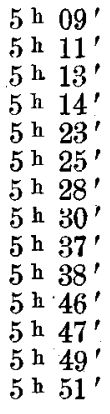 & $\begin{array}{l}190 \\
190 \\
190 \\
190 \\
190 \\
190 \\
190 \\
190 \\
190 \\
190 \\
190 \\
190 \\
190 \\
190\end{array}$ & $\begin{array}{l}10 \\
10 \\
10 \\
10 \\
10 \\
10 \\
10 \\
10 \\
10 \\
10 \\
10 \\
10 \\
10\end{array}$ & $\begin{array}{l}25 \\
20 \\
20 \\
-20 \\
-- \\
- \\
14 \\
3 \\
2 \\
2 \\
3\end{array}$ & $\begin{array}{c}\overline{-} \\
\text { Depression } \\
\begin{array}{c}- \\
\text { Depression } \\
\text { Depression }\end{array} \\
- \\
- \\
22 \\
- \\
-\end{array}$ & $\begin{array}{l}5 \text { ccm Jodothyrinlösung } \\
\quad(=1,5 \mathrm{mg} \mathrm{J} .) .\end{array}$ \\
\hline
\end{tabular}


Die Ansprechbarkeit sowohl des linken wie des rechten Vagus steigt nach Jodothyriuzufubr. Vgl. Fig 37 und 38.

Nun wurde der Einfluss des Jodothyrins auf den Adrenalineffekt geprüft.

Tabelle 38.

Versuch XVIII. 5. Juli 1913. Kaninchen, Gewicht 2150 g. $91 / 2$ Uhr $2 \mathrm{~g}$ Urethan. Vagi und Depressoren beiderseits durchschnitten. $0,5 \mathrm{ccm}$ Adrenalinlösung $={ }^{1 / 100} \mathrm{mg}$ Substanz.

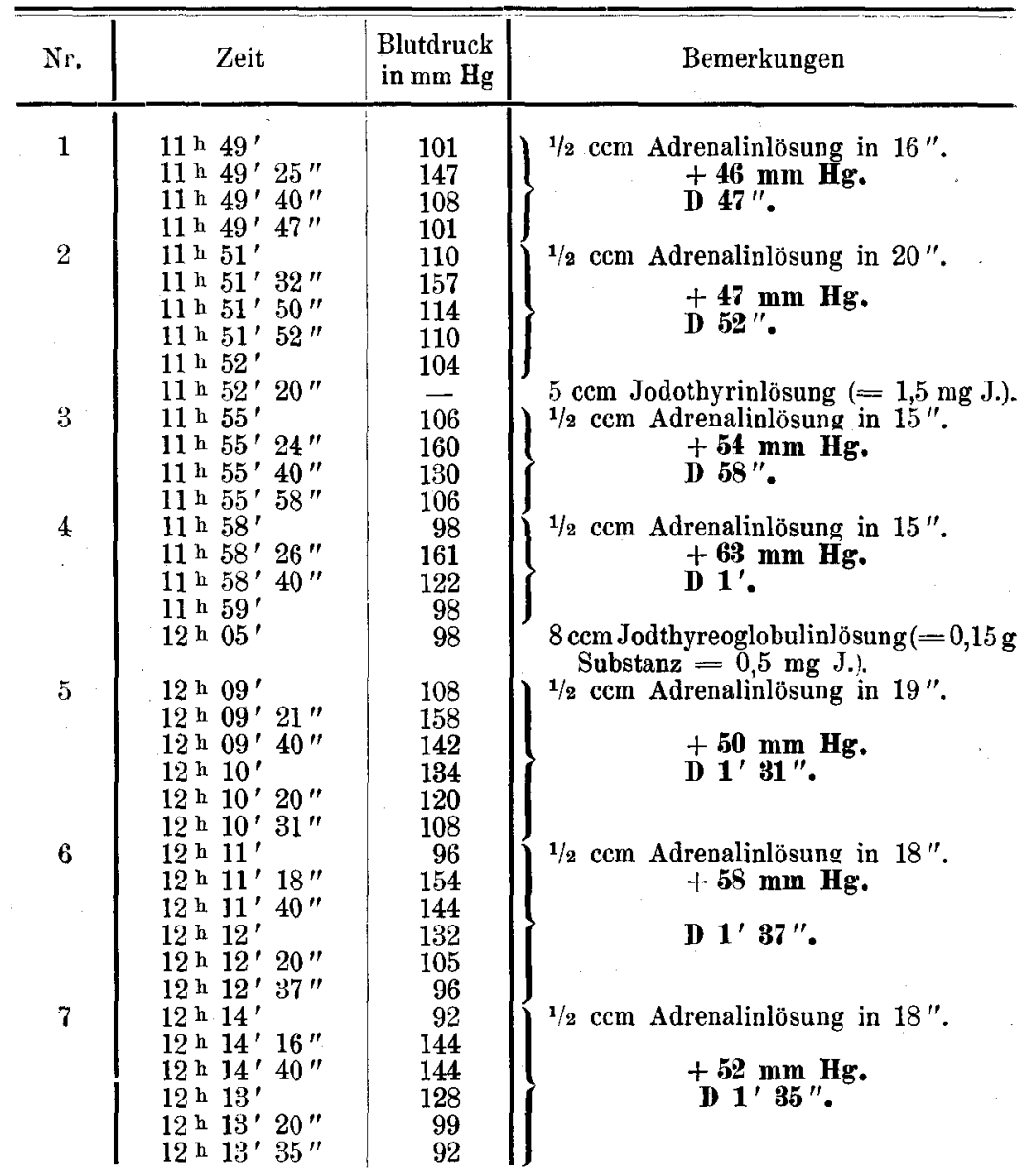

Jodothyrin bewirkt eine geringe Steigerung des Adrenalineffektes, die nachher, was wenigstens die Dauer des Effektes anbelangt, durch Jodthyreoglobulin vermehrt wird. Bei der 
Beurteilung der Höhe der Blutdrucksteigerung ist hier wie in allen ähnlichen Versuchen zu bedenken, dass sie bei mehrmals wiederholter Injektion allmählich abzunehmen beginnt (Adrenalinmüdigkeit). Der Umstand, dass dies hier nicht zu beobachten ist, spricht schon allein für eine Zunahme der Ansprechbarkeit unter dem Einfluss des Jodthyreoglobulins. $\mathrm{Zu}$ beachten ist, dass mit dem Jodothyrin dreimal mehr Jod eingeführt wurde als mit dem Jodthyreoglobulin (1,5 $\mathrm{mg}$ gegen 0,5$)$.

Die gleiche Wirkung des Jodothyrins auf die Adrenalinempfindlichkeit ergibt sich auch aus den weiter unten zu schildernden Versuchen XXXIII und XXXIV (Tab. 41 und 42). Siehe diese.

Aus diesen Versuchen geht hervor, dass Jod othyrin im gleichen Sinne wirksam ist auf die Ansprechbarkeit des Vagus und Depressorsund auf dieAdrenalinempfindlichkeit wie seine Muttersubstanz, doch schwächer auf den Jodgehalt bezogen als diese. $0,06 \mathrm{~g}$ Jodothyrin, enthaltend 1,5 mg Jod, hat einen geringeren Effekt als $0,15 \mathrm{~g}$ Jodthyreoglobulin mit $0,5 \mathrm{mg}$ Jod. Das Jod macht somit nicht alles aus, es kommt noch auf das übrige Molekül an.

Die relativ geringere Wirksamkeit des Jodothyrins im Vergleich zum Jodthyreoglobulin, auf den Jodgehalt bezogen, macht es unwahrscheinlich, dass die Wirkung einfach der organischen Bindung des Jods zuzuschreiben sei. Dennoch sollte geprüt werden, wie sich kunstlich jodiertes Eiweiss verhielt. Es musste das um so mehr geschehen, als v. Fürth und Schwarz das Jodothyrin mit jodhaltigen Melanoidinen identifizieren. Weiterhin sollte auch geprüft werden, wie sich i onisiertes Jod verhält.

Als künstlich jodiertes Eiweiss verwendete ich J odkas ein, das ich nach dem von mir angegebenen Verfahren ${ }^{1}$ ) darstellte. Das so gewonnene Präparat hat den Vorteil, ganz farblos zu sein und es auch bei sehr langem Aufbewahren selbst am Tages- und Sonnenlicht zu bleiben. Ich besitze seit mehreren Jahren Präparate, die vollkommen farblos sind. Weiterhin verwendete ich Jod ty rosin, als ein im Jodeiweiss vorkommender Komplex ${ }^{1}$ ). Als ionisiertes Jod wurde Jodnatrium verwendet. Es folgen die Versuchsprotokolle.

1) A. Oswald, Gewinnurg von 3,5-Dijodtyrosin aus Jodeiweiss. Zeitschr. f. physiolog. Chemie, Bd. 74 S. 290. 1911 Vgl. auch: Derselibe, Notiz über Jodeiweiss. Zeitschr. f. physiolog. Chemie Bd. 95 S. 351. 1915. 
Tabelle 39.

Versuch VIII. 29. Mai 1913. Kaninchen, Gewicht 2000 g. 31/2 Uhr $1 \mathrm{~g}$ Urethan subkutan. $5 \mathrm{Uhr}$ aufgespannt. Jodkaseinlösung enthält $1 \% \mathrm{Jod}$ kasein und $1 \%$ Soda.

\begin{tabular}{|c|c|c|c|c|c|c|}
\hline \multirow[t]{2}{*}{$\mathrm{Nr}$. } & \multirow[t]{2}{*}{ Zeit } & \multirow{2}{*}{$\begin{array}{l}\text { Rollen- } \\
\text { abstand } \\
\text { in } \mathrm{mm}\end{array}$} & \multirow{2}{*}{$\begin{array}{l}\text { Dauer } \\
\text { d. Rei- } \\
\text { zung } \\
\text { in Sek. }\end{array}$} & \multicolumn{2}{|c|}{$\begin{array}{l}\text { Pulszahl in } 10 \text { Sek. } \\
\text { bei Reizung des }\end{array}$} & \multirow[t]{2}{*}{ Bemerkungen } \\
\hline & & & & 1. Vagus & r. Vagus & \\
\hline 1 & $5^{\text {h }} 12^{\prime}$ & 190 & 10 & 12 & $\ldots$ & \multirow{7}{*}{$\begin{array}{l}4 \text { ccm Jodkaseinlösung } \\
=0,04 \mathrm{~g} \text { Substanz } \\
=5 \mathrm{mg} \text { J.). }\end{array}$} \\
\hline \multirow[t]{2}{*}{$\begin{array}{l}1 \\
2\end{array}$} & $5^{\text {h }} 16^{\prime}$ & 190 & 10 & - & 16 & \\
\hline & 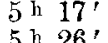 & 100 & $\overline{10}$ & $\overline{15}$ & - & \\
\hline 4 & 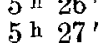 & $\begin{array}{l}190 \\
190\end{array}$ & $\begin{array}{l}10 \\
10\end{array}$ & 15 & $\overline{16}$ & \\
\hline 5 & $5^{\mathrm{h}} 37^{\prime}$ & 190 & 10 & $\overline{26}$ & - & \\
\hline 6 & 5 h $39^{\prime}$ & 190 & 10 & 22 & 一 & \\
\hline 7 & $5 \mathrm{~h} 50^{\prime}$ & 190 & 10 & 19 & 一 & \\
\hline
\end{tabular}

Jodkasein erhöht somit die Ansprechbarkeit des Vagus gegenüber dem faradischen Strom nicht.

Nun wurde ionisiertes Jod auf seine Wirksamkeit geprüft.

$$
\text { Tabelle } 40 .
$$

Versuch XI. 10. Juni 1913. Kaninchen, Gewicht 2550 g. 3 Uhr 2 g Urethan subkutan. Jodnatriumlösung $=5 \%$ ig.

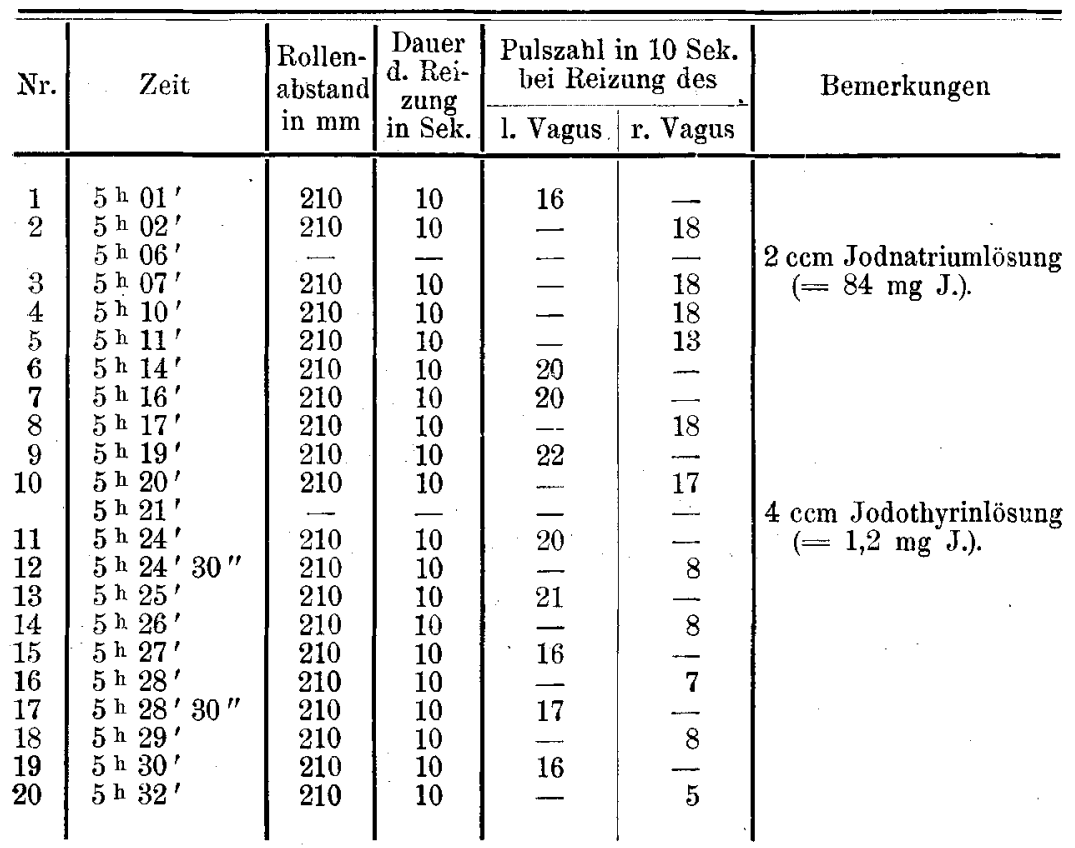


Die Pulszahl bleibt nach Jodnatriumzufuhr die gleiche wie vorher. Erst Jodothyrinbeibringung bringt sie zum Sinken. Dabei ist $\mathrm{zu}$ beachten, dass mit dem Jodnatrium 35 mal mehr Jod eingeführt worden ist als mit dem Jodothyrin.

v. Cyon ${ }^{1}$ ) nimmt zwar an, indem er sich auf Untersuchungen عeines Schülers Barbera ${ }^{2}$ ) stützt, dass Jodnatrium die Erregbarkeit des Vagus und Depressors für den faradischen Strom herabsetzt. Die von Barbera seinen Versuchstieren beigebrachten Jodmengen sind aber enorm gross (bis 3,2 g Jodnatrium auf einmal beim Kaninchen und der Katze). Es frägt sich, ob man das Recht hat, aus solchen Versuchen Schlüsse zu ziehen. Auch sind die konfus und unübersichtlich geschilderten Versuche nichts weniger als einwandfrei. Ausserdem hat schon $\mathrm{Harnack}^{3}$ ) darauf hingewiesen, dass bei dieser hohen Dosierung eine Wirkung der Na-Ionen auf den Herzmuskel in Betracht zu ziehen ist, welche den von v. Cy on für das Jod angenommenen Effekt vortäuschen könnte. Die Na-Ionen erhöhen nämlich den Muskelreiz und an einem unter solchen Umständen arbeitenden Herzen ist eine Vagusreizung erfolglos.

Tabelle 41.

Versuch XXXIV. 22. Dezember 1913. Kaninchen, Gewicht 1900 g. Crethan subkutan. Vagi und Depressoren intakt.

\begin{tabular}{|c|c|c|c|}
\hline Nr. & Zeit & $\begin{array}{r}\text { Blutdruck } \\
\text { in } \mathrm{mm} \mathrm{Hg}\end{array}$ & Bemerkungen \\
\hline 1 & $\begin{array}{l}6^{\text {h }} 18^{\prime} \\
6^{\text {h }} 18^{\prime}, 13^{\prime \prime} \\
6 \text { h } 18^{\prime} 50^{\prime \prime} \\
6^{\text {h }} 19^{\prime}, 20^{\prime \prime} \\
6 \text { h } 20^{\prime} \\
6 \text { h } 20^{\prime} 18^{\prime \prime} \\
6 \text { h } 20^{\prime}, 53^{\prime \prime} \\
6 \text { h } 21,15^{\prime \prime} \\
6 \text { h } 23^{\prime} \\
6^{\text {h h }} 23^{\prime} 20^{\prime \prime}\end{array}$ & $\begin{array}{r}72 \\
125 \\
72 \\
67 \\
64 \\
66 \\
117 \\
66 \\
66 \\
68\end{array}$ & 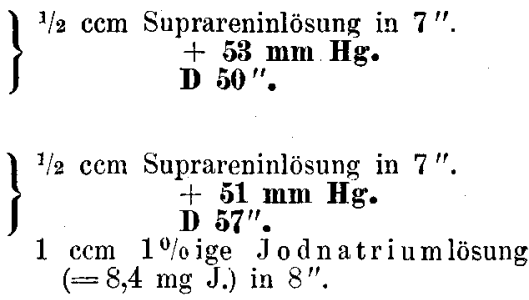 \\
\hline
\end{tabular}

1) E. v. Cyon, Beiträge zur Physiologie der Schilddrüse und des Herzens. Dieses Arch. Bd. 70 S. 126. 1898. - E. v. Cyo n, Die physiologischen Herzgifte. Dieses Arch. Bd. 73 S. 42 u. 339.1898.

2) A. G. Barbera, Über die Erregbarkeit von Herz- und Gefässnerven nach Injektion von Jod und phosphorsaurem Natron. Dieses Arch. Bd. 68 S. 434 . 1897 .

3) loc. cit. 
Tabelle 41 (Fortsetzung).

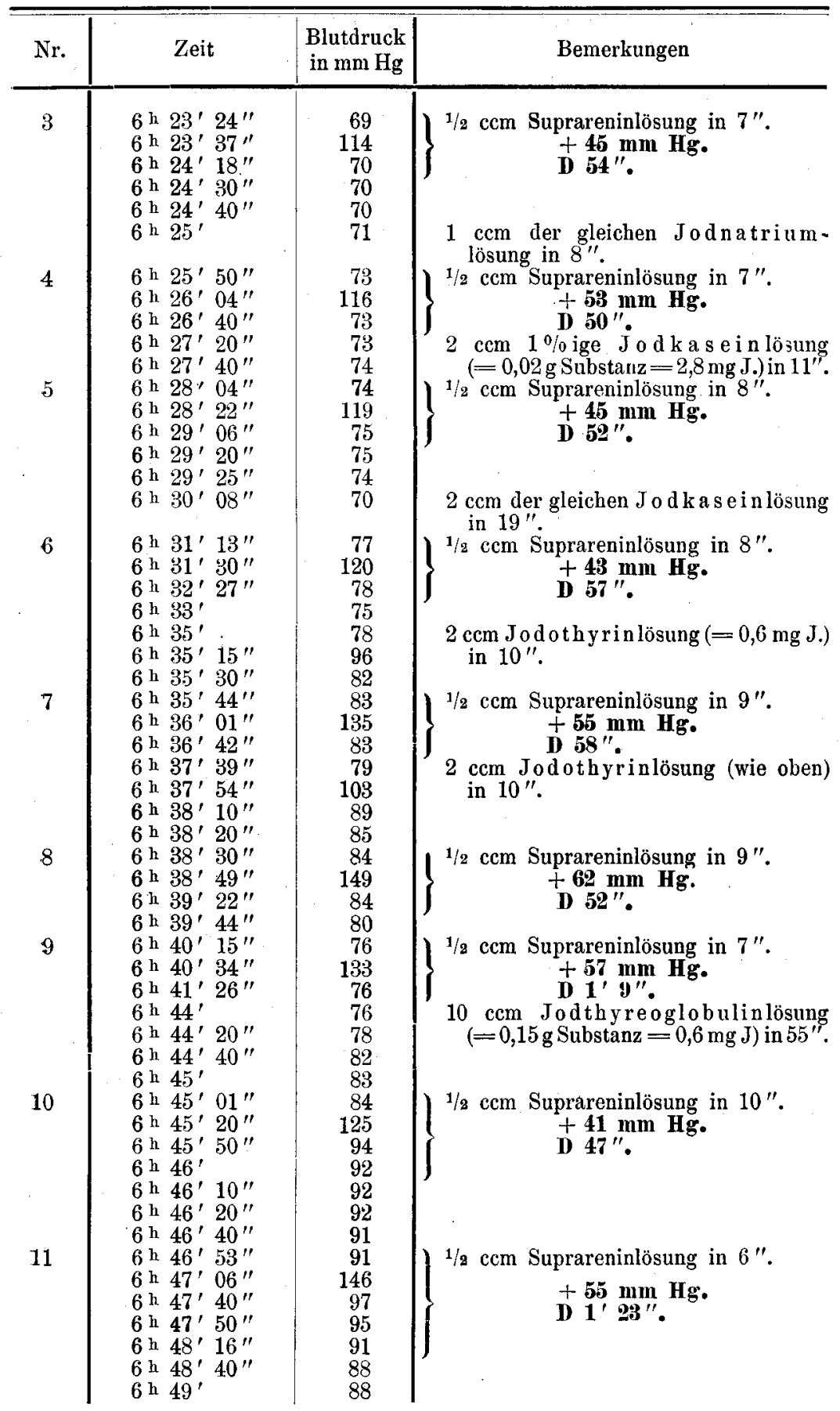


Aus diesem Versuch geht hervor, dass Jodnatrium und Jodkasein auf die Adrenalinempfindlichkeit unwirksam sind. Dagegen erweist sich Jodothyrin als wirksam, wenn auch schwächer als Jodthyreoglobulin. Der zweite Teil des Versuches dürfte nicht mehr so maassgebend sein, da durch die wiederholten Adrenalininjektionen bereits Adrenalinmüdigkeit eingetreten ist. Darum zeigt auch Jodthyreoglobulin keine so starke Wirksamkeit mehr.

Tabelle 42.

Versuch XXXIII. 9. Dezember 1913. Kaninchen, Gewicht 2200 g. Urethan. Vagi und Depressoren intakt.

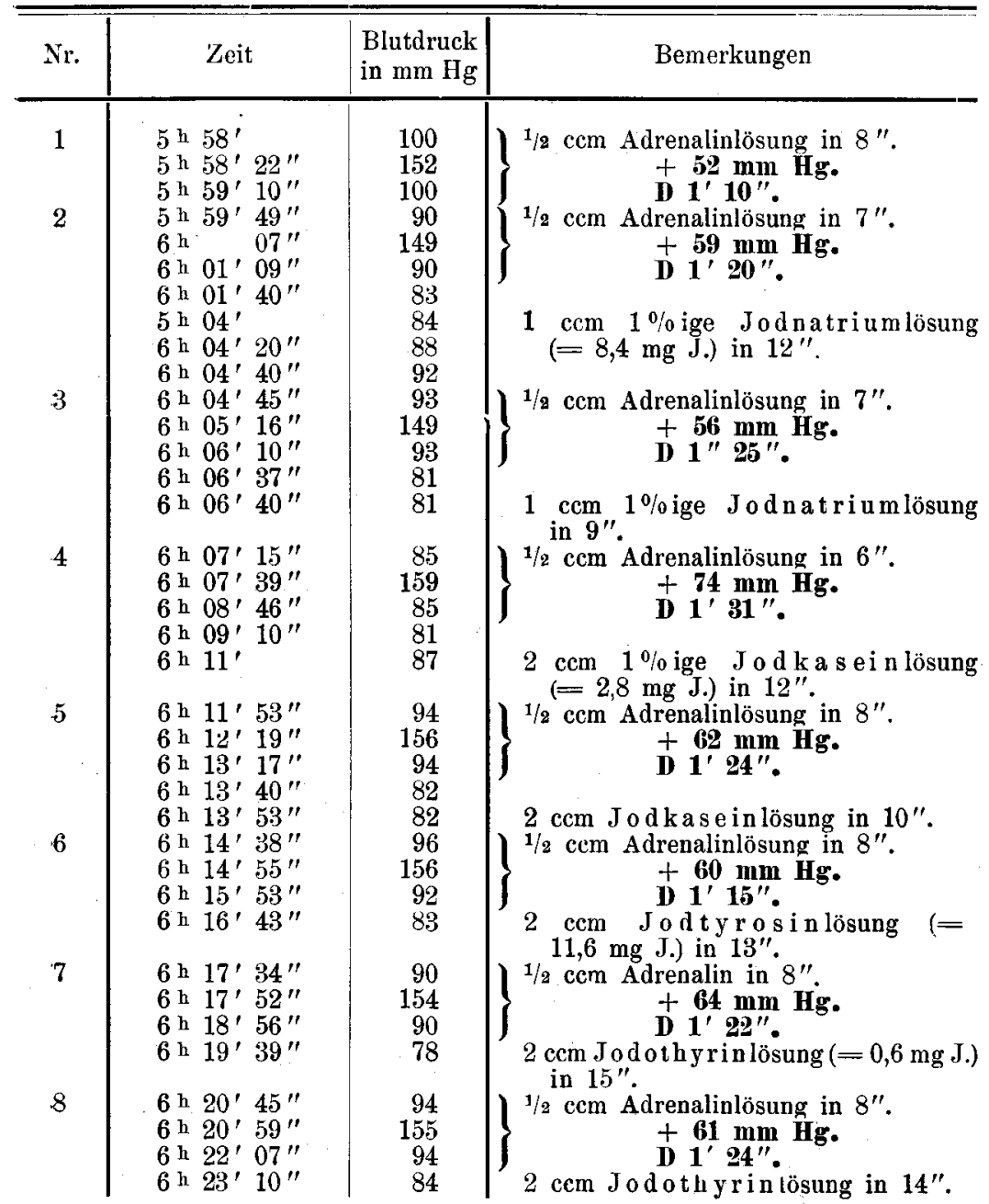


Tabelle 42 (Fortsetzung).

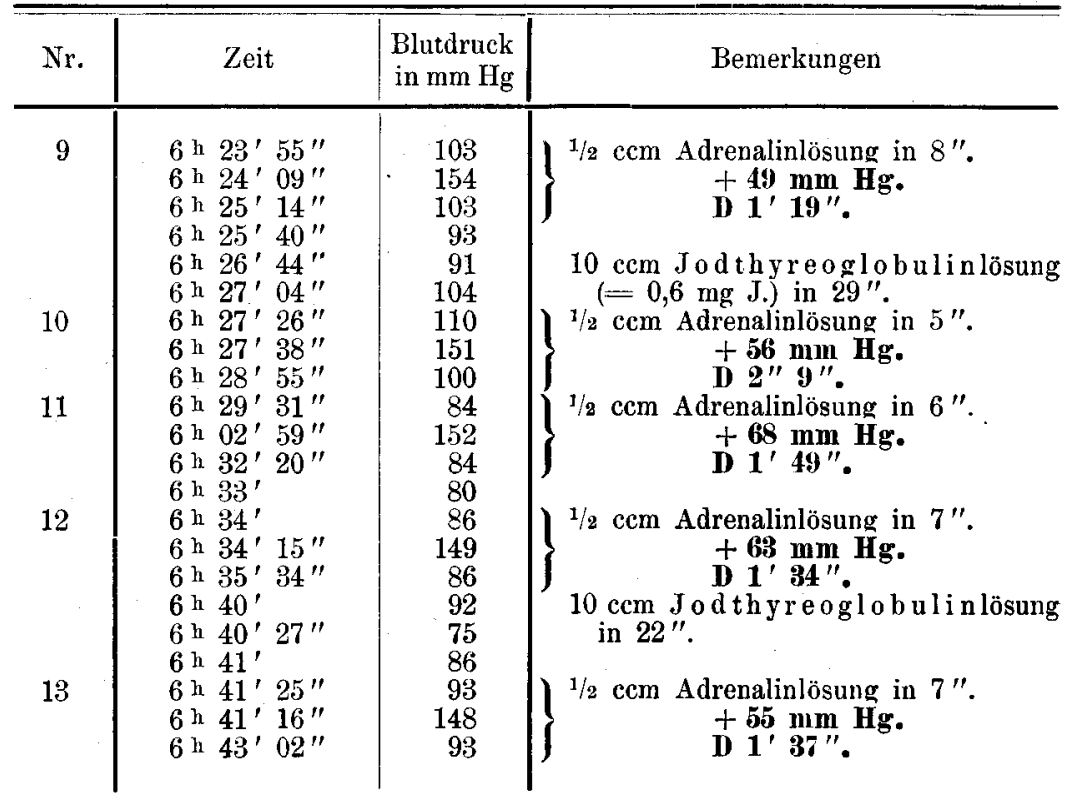

Aus diesem Versuch ergibt sich, dass Jodnatrium, Jodkasein und Jodtyrosin auf die Adrenalinempfindlichkeit unwirksam sind. Auch Jodothyrin erweist sich hier als unwirksam. Erst Jodthyreoglobulin bewirkte eine beträchtliche Verlängerung der Blutdrucksteigerung. $\mathrm{Zu}$ beachten ist, dass mit jeder Jodnatrium-, Jodkasein- und der Jodtyrosindosis: 14- bzw. 4- und 19 mal mehr Jod eingeführt wurde als mit dem Jodothyrin bzw. Jodthyreoglobulin. Insgesamt wurden mit dem Jodnatrium, Jodkasein und Jodtyrosin $24 \mathrm{mg}$ Jod eingeführt, also 40 mal mehr als mit Jodothyrin bzw. Jodthyreoglobulin. Mit Jodothyrin wurde die gleiche Menge Jod eingeführt wie mit. Jodthyreoglobulin $(0,6 \mathrm{mg})$, trotzdem erwies sich letzteres als wirksam und ersteres nicht. Nach der elften Adrenalininjektion nahm der Effekt wieder ab, trotz Jodthyreoglobulinwirkung. Hier macht sich Adrenalinmüdigkeit bemerkbar.

Aus all diesen Versuchen ergibt sich, dass Jod in Gestalt von Jodid, Jodkasein und Jorltyrosin nicht die Wirksamkeit des Jodthyreoglobulins auf die Vagusansprechbarkeit und die Adrenalinempfindlichkeit der peripheren Gefässwände hat. Jodothyrin besitzt sie, jedoch auf den Jodgehalt bezogen, in schwächerem Maasse. Daraus ergibt sich, dass Jod an und für sich ebensowenig wie die 
blosse Tatsache seiner organischen Bindung die ausreicheude Ursache der Jodthyreoglobulinwirksamkeit ist, d. h. alșo dass auch das übrige Molekül für diese verantwortlich zu machen ist.

\section{Prüfung der Wirksamkeit des Jodthyreoglobulins aus pathologisch veränderten Schilddrüsen.}

Endlich sollte die für die Pathologie wichtige Frage gelöst werden, wie sich Jodthyreoglobulin aus pathologisch veränderten Schilddrüsen verhielt. Das Verhalten einfacher hyperplastischer und Kolloidstrumen ist im vorhergehenden schon besprochen worden. Als ausschlaggebend hat sich die Höhe des Jodgehaltes erwiesen. Fin weiterer Unterschied konnte nicht konstatiert werden. Es frug sich nun, wie sich zum Beispiel das Jodthyreoglobulin aus Basedowschilddrüsen verhielt. Mit Rücksicht auf die bei dieser Krankheit vorkommenden Zirkulationsstörungen, insbesondere die ausserordentlich gesteigerte Herzaktion musste entschieden werden, ob sich ein verschiedenes Verhalten nach dieser Richtung auch im Tierversuch unter den gewählten Versuchsbedingungen nachweisen liesse. Damit liess sich in einer gewissen Hinsicht die alte Streitfrage entscheiden, ob eine Dys-oder Hy perthy reose das Wesentliche bei dieser Erkrankung sei. Ein weiterer der Prüfung werter Gegenstand ist das Verhalten von aus Kretinenschilddrüsen dargestelltem Jodthyreoglobulin: bei der Ähnlichkeit dieses Zustandes mit Myxödem musste sich die Notwendigkeit der Untersuchung von selbst aufdrängen. Von vornherein konnte keine Abweichung nach bestimmter Richtung erwartet werden, da die Verhältnisse beim Kretinismus jedenfalls nicht so einfach liegen wie beim Myxödem. Um so mehr lohnte sich eine Prüfung.

Kretinismus hat mit Myxödem klinisch vielfach Ähnlichkeit und muss mit ihm in die Kategorie der Hyperthyreosen gestellt werden. Auch lässt sich mit Jodthyreoglobulin Kretinismus wesentlich bessern, in vielen Fällen praktisch sogar heilen ${ }^{1}$ ). Im Gegensatz zu den Myxödematösen haben aber die Kretinen eine Schilddrüse ${ }^{2}$ ) und oft

1) Vgl. meine Mitteilung: Zur Behandlung des endemischen Kretinismus. Korrespondenzbl; f. Schweizer Ärzte 1914 Nr. 24.

2) Die besten Heilerfolge mit Jodthyreoglobulin habe ich erzielt bei solchen, welche keinen Kropf, überhaupt keine palpatorisch nachweisbare Schilddrüse haben.

Pflüger's Archiv für Physiologie. Bd. 164. 
genug einen Kropf. Man könnte daher a priori vermuten, dass diese Kröpfe kein physiologisch wirksames Sekret lieferten. Dem stellt sich aber wieder die Tatsache entgegen, dass ein Kretin, dem der Kropf total exstirpiert wurde, Myxödem bekam ${ }^{1}$ ). Es bliebe also nur der Ausweg offen, dass der Kretinenkropf aus irgendeinem Grunde verhindert sei, sein Sekret an den Körper abzugeben. Doch unterscheidet sich der Kretinenkropf pathologisch-anatomisch prinzipiell nicht von dem gewöhnlichen Kolloidkropf, so dass für diese Annahme auch kein tatsächlicher Untergrund bleibt. Aus diesem kurzen Überblick ${ }^{2}$ ) ist jedenfalls zu ersehen, dass die ursächlichen Beziehungen zwischen Kropf und Kretinismus nicht so einfacher Natur sind wie die zwischen dem Fehlen von Thyreoidea und dem Vorkommen von Myxödem; um so mehr ist jeder Versuch angebracht, der irgendwelchen Anhaltspunkt zu einer befriedigenden Aufklärung beibringen kann. Aus diesem Gruncle war es meine Absicht, die physiologische Prüfung von Thyreoglobulin aus Kretinenkröpfen vorzunehmen.

Leider ist es mir bisher nicht möglich gewesen, frische Schilddrüsen oder Kröpfe von Kretinen zu erhalten. und ich muss mir die Prüfung des Sekretes aus Drüsen solcher Kranken auf später vorbehalten. Dagegen habe ich Jod thy reoglobulin aus Basedow drüsen der Prüfung unterzogen.

Die Basedowstrumen stammten von Kranken, welche die Krankheit in ausgesprochener Weise zeigten, insbesondere wiesen sie Exophthalmus, Tachykardie, die Liảsymptome und Tremor auf. Bei der Nekropsie zeigten die Strumen den Adspekt von Kolloidkröpfen.

Ich lasse nunmehr in Tabelle 43 (S. 575) einen Protokollauszug folgen.

Aus dem Versuch ergibt sich, dass Jodthyreoglobulin aus Basedowstrumen auf den blutpressorischen Adrenalineffekt den gleichen Einfluss hat wie dasjenige aus gesunden Drüsen, und dass die Intensität dieses Einflusses auch annähernd die gleiche ist. $\mathrm{Zu}$ bemerken ist, dass die nach dem Abklingen der Adrenalinwirkung

1) H. Bircher, Das Myxödem und die kretinische Degeneration. Volkmann's Klin. Vorträge Nr. 857. 1890.

2) Eine ausführliche Darlegung dieser und aller übrigen einschlägigen Verhältnisse findet der Leser in meiner vor kurzem erschienenen monographischen Darstellung: Die Schilddrüse in Physiologie und Pathologie. Veit \& Co., Leipzig 1916. 
Tabelle 43 .

Versuch XXVI. 13. November 1913. Kaninchen, Gewicht 2980 g. Vagi und Depressoren intakt. Die Adrenalinlösung enthält $1 \mathrm{mg}$ Substanz auf $50 \mathrm{ccm} 0,9 \%$ iger Kochsalzlösung. Das verwendete Jodthyreoglobulin enthielt $0,13 \% \mathrm{~J}$.

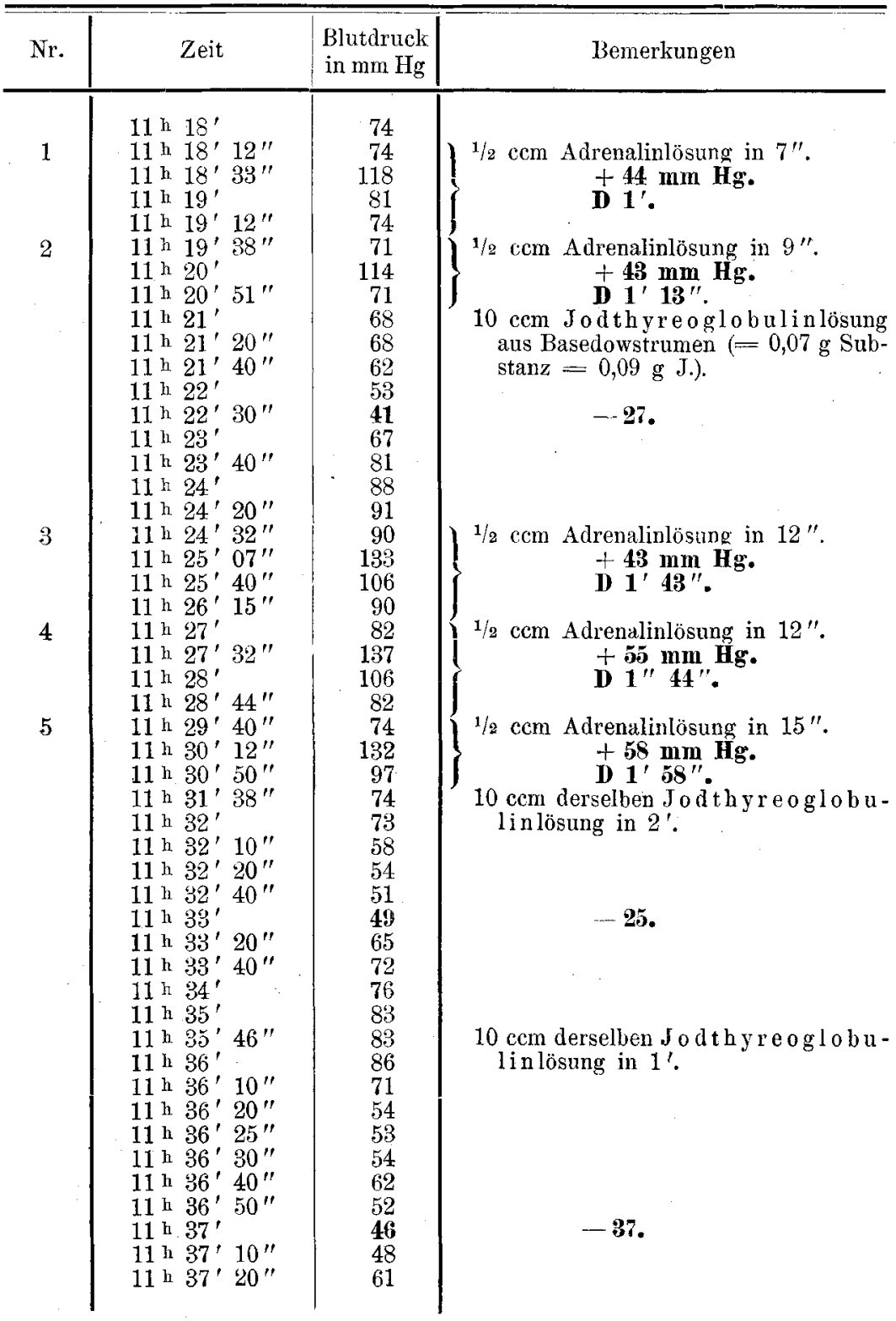


Tabelle 43 (Fortsetzung).

\begin{tabular}{|c|c|c|c|}
\hline $\mathrm{Nr}$ & Zeit & $\begin{array}{l}\text { Blutdruck } \\
\text { in } \mathrm{mm} \mathrm{Hg}\end{array}$ & Bemerkungen \\
\hline 6 & 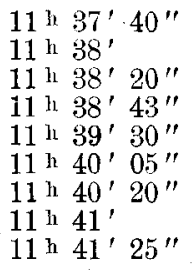 & $\begin{array}{r}75 \\
80 \\
83 \\
86 \\
129 \\
115 \\
110 \\
86 \\
84\end{array}$ & $\begin{array}{l}\text { 1/2 ccm Adrenalinlösung in } 20 " . \\
+\mathbf{4 3} \mathbf{~ m m} \mathbf{H g} \text {. } \\
\text { D } \mathbf{2}^{\prime} \mathbf{1 7}{ }^{\prime \prime}\end{array}$ \\
\hline
\end{tabular}

verursachte Blutdruckdepression sehr ausgesprochen ist. Aus diesem einen Versuch darf natürlich kein genereller Schluss gezogen werden. Die Tatsache soll denn auch nur registriert und weitere Beobachtungen abgewartet werden. Das gleiche Präparat bewirkte vor der Adrenalinwirkung keine Blutdruckveränderung.

\section{Zusammenfassende Betrachtung der. gewonnenen Resultate.}

Die im vorhergehenden niedergelegten Beobachtungen sind im Verein mit anderen, noch gleich zu erwähnenden Erhebungen geeignet, uns ein Bild über die physiologisehe Bedeutung der Schilddrüse zu geben. Es geht aus dem Mitgeteilten hervor, dass sie ein Organ ist, dessen Sekret die Ansprechbarkeit des Vagus, des Depressors und des Splanchnicus für den faradischen Strom und, was letzteren Nerven anbelangt, auch für ein pharmakodynamisches Mittel (das Adrenalin) steigert. Daraus köunen wir schliessen dass der Tonus dieser Nerven durch das Jodthyreoglobulin erhöht wird. Diese Schlussfolgerung ergibt sich zwar nicht unmittelbar aus den erwähnten Beobachtungen, indem auch bloss die Erfolgsorgane stärker ansprechen könnten. Doch haben wir Grund, namentlich mit Rücksicht auf die gleich anzuführenden Beobachtungen am Zentralnervensystem, eine Erhöhung des Nerventonus anzunehmen. Wie- sich übrigens aus einer späteren Mitteilung ergeben wird, werden auch andere Effekte des Adrenalins, so zum Beispiel seine thermische Wirkung in förderndem Sinne beeinflusst ${ }^{1}$ ). Wir können somit sagen, dass das Jodthyreoglobulin den Tonus ausgedehnter Gebietedes autonomen (parasympathischen)

1) Diese Befunde sollen den Gegenstand einer anderen Mitteilang bilden. 
wie des sympathischen Nervensystemserhöht. Nun tun Beobachtungen anderer Art, die ich an anderem Orte ausführlich geschildert habe ${ }^{1}$ ), ausserdem dar, dass nicht nur das vegetative, sonclern auch das animale (Zentral-) Nervensystem durch das Jodthyreoglobulin ansprechbarer gemacht wird. Das kommt zum Ausdruck durch ehronische Zufuhr von Schilddrüsensubstanz bzw. Jodthyreoglobulin, mitunter aber auch bei besonders disponierten Individuen nach nur wenigen und geringen Dosen davon. Solche Menschen bekommen nach Jodthyreoglobulinzufuhr allgemeine Nervosität, motorische Unruhe, gesteigerte Reflexerregbarkeit, Insomnie, Tremor der Hände, der Zunge, psychische Depression usw., kurz, Zeichen erhöhter Irritabilität des zerebrospinalen Nervensystems. Auch von Tieren ist das bekannt. Wir sind somit zu der Behauptung berechtigt, dass das Jodthyre oglobulin eineden Nerventonus im allgemeinen in exquisiter Weise erhöhende Substanz darstellt. Da die Schilddrüse unter dem Einfluss des Nervensystems steht und von ihm aus auf dem Wege der Nn. laryngei ihre Impulse zur Sekretion erhält, so ergibt sich des weiteren, dass sie, physiologisch gesprochen, ein dem Nervensystem interpoliertes Organ darstellt, das dem Zwecke dient, gewissen von ihm ausgehenden Reizen mehr Nachachtung zu verschaffen. Denn sie empfängt von ihm Reize, und durch Vermittlung ihres Sekretes ermöglicht sie dem Nervensystem seine Reizwirkungen zu verstärken. Sie spielt somit die Rolle eines Multiplikators der Nervenreize.

Die Schilddrüse übt, wie wir schon lange wissen, einen ausgesprochenen Einfluss im Sinne einer Steigerung auf die Stoffwechselvorgänge aus. Der Sauerstoffverbrauch, die Kohlensäureproduktion und die Stickstoffausscheidung werden vermehrt. Sie bewirkt einen Körpergewichtsverlust. Wie ich an der Hand von Beobachtungen an Menschen, die an Hyperthyreoidismus litten, dargetan habe ${ }^{1}$, ist nun dieser Einfluss in besonderem Maasse ausgeprägt bei Individuen mit leicht ansprecbbarem Nervensystem. Dort kann die Abmagerung in der kürzesten

1) A. Oswald, Über die Gefahren der Jodmedikation. Deutsch. Arch. f. klin. Med. Bd. 117 S. 551. 1915. - A. Os wald, Über die Rolle des Nervensystems in der Genese der Stoffwechsel- und Konstitutionskrankheiten. Neurol. Zentralbl. 1915 Nr. 19. 
Zeit ausserordentliche Dimensionen annehmen, es erfolgt rapider Fett- und auch Eiweisszerfall. Es kann in 14 Tagen ein Körpergewichtsverlust von $20 \mathrm{~kg}$ eintreten bei unveränderter, ja gesteigerter Nahrungszufuhr, während Menschen mit nicht geschwächtem Nervensystem in dieser Zeit von der gleichen Dosis Schilddrüsensekret einen kaum merklichen Gewichtsverlust aufweisen. Das drängt natürlich die Frage auf die Lippen, ob nicht auch die Oxydationen und im weiteren auch die übrigen Stoffwechselvorgänge weit mehr unter dem Einfluss des Nervensystems stehen, als man gemeiniglich annimmt, und ob somit die Wirkung des Schilddrüsensekretes auf den Stoffwechsel nicht ibren Weg über das Nervensystem nimmt. Damit würde unsere Vorstellung über die physiologische Arbeit der Schilddrüse an Einheitlichkeit gewinnen. Sie würde sich ganz allgemein erweisen als bestehend in einer Förderung des Nervensystems (des animalen und vegetativen) im Sinne einer Steigerung seiner Ansprechbarkeit gegen äussere und innere Reize.

Eine Abhängigkeit der Stoffwechselvorgänge vom Nervensystem tritt uns bekanntlich als Postulat auch aus Beobachtungen der menschlichen Pathologie entgegen.

Eine besondere Empfindlichkeit gegenüber dem Jodthy reoglobulin findet sich bei manchen Menschen nicht nur bezüglich der Stoffwechselvorgänge; die gleichen Individuen sind äuch bezüglich ihrer Kreislauforgane resp. der dieselben versorgenden Nerven (wie überhaupt des gesamten Nervensystems) ausserordentlich empfindlich. Damit komme ich auf einen Punkt zu sprechen, der für die Beurteilung der Befunde mancher Experimentatoren von Bedeutung ist. Wir haben gesehen, dass manche Experimentatoren nach Schilddrüsenzufuhr im Gegensatz zu den meisten anderen Pulsbeschleunigung und Blutdruckerhöhung beobachtet haben. Prägnante Beispiele sind die von Hein $\operatorname{tz}^{1}$ ). Da diese Abhandlung russisch verfasst ist, dürfte sie den meisten Lesern deutscher Zunge im Original nicht zugänglich sein. Ich zitiere darum einen besonders instruktiven Passus daraus, den ich einer Arbeit Georgiewsky's²) entnehme. Er lautet: "Bei allen Hunden ohne Ausnahme hob sich der Blutdruck merklich, unmittelbar nach jeder Injektion, war ziemlich lange erhöht und

1) Heinatz, Altes und Neues über die Schilddrüse. Inaug.-Diss. (Russ.) 1894.

2) loc. cit. 
sank dann langsam bis zur Norm herab. Der Erhöhung des Blutdruckes ging eine bedeutende Beschleunigung der Herzkontraktionen voraus, welche einige Zeit andauerte und verschwand, bevor das Sinken des Blutdruckes eintrat. Diese beiden Erscheinungen fallen nie zusammen, sondern immer geht die Beschleunigung des Pulses der Erhöhung des Blutdruckes voran und hört früher auf als diese."

Der Autor führt folgenden Versuch an: "Vor der Injektion ist der Blutdruck $80 \mathrm{~mm}$, der Puls 96 in der Minute. 11/2 Stunden nach einer Injektion von $10 \mathrm{ccm}$ Schilddrüsensaft stieg der Blutdruck auf $152 \mathrm{~mm}$, und die Zabl der Herzkontraktionen erreichte 224 in der Minute. Noch $1 / 2$ Stunde später stieg der Blutdruck his $164 \mathrm{~mm}$, die Zahl der Kontraktionen aber begann zu fallen und betrug 150 in der Minute; allmählich fing auch der Blutdruck zu sinken an. Die Durchschneidung der Nn. vagi hatte keinen Einfluss auf den Ausgang des Experimentes. Beschleunigung des Pulses und Erhöhung des Blutdruckes traten auch nach der Durchschneidung derselben ein ..."

Mit diesem Befund stellt sich Heinatz in Gegensatz zu den meisten anderen Experimentatoren (vgl, weiter oben die entsprechenden Abschnitte). Das will jedoch keineswegs besagen, dass wir an dessen Richtigkeit zweifeln sollen. Die Angaben sind zu bestimmt dazu. Die Erklärung liegt vielmehr anderswo.

Wir haben gesehen, dass manche Menschen gegenüber dem Jodthyreoglobulin sehr empfindlich sind. Solche Individuen bekommen nach Genuss von Schilddrüsensubstanz, oft auch schon nach geringen Dosen und nur einige Male wiederholter Zufuhr oder auch durch eine irgendwie bewirkte rasche Verkleinerung der Schilddrüse (des Kropfes) - durch Jodbehandlung oder Röntgenbestrahlung - die klinischen Symptome des Hyperthyreoidismus ${ }^{1}$ ). Die Symptome seitens des Kreislaufs, die uns hier ausschliesslich interessieren, sind Tachykardie, Herzklopfen, Aktionspulse, Dermographismus. Die Ursache des Auftretens dieser Symptome liegt in einer grossen Labilität des Nervensystems, speziell des viszeralen, denn Menschen mit gesunden Nerven zeigen sie nicht, selbst nach dem Genuss relativ grosser Dosen von Schildrüsensubstanz resp. Jodthyreoglobulin ${ }^{2}$ ).

1) Siehe weiter oben.

2) Adolf Oswald, Zur Theorie des Basedow. Münchener med. Wochenschrift 1915 Nr. 27. 
Wenn nun bei Tieren die Mehrzabl auf Schilddrüsensubstanz resp. Jodthyreoglobulin, besonders im akuten Experiment, von dem hier ausschliesslich die Rede ist, nicht besonders stark anspricht und keine Pulsbeschleunigung und Blutdruckerhöhung bekommt, wohl aber die Minderzahl, so liegt es nahe, auch da eine besondere Labilität des Nervensystems anzunehmen. Daran ändert die Tatsache nichts, dass ganze Tierrassen eine grössere Ansprechbarkeit zeigen, unter den Hunden zum Beispiel die Foxterriers: wissen wir ja, dass gerade diese Rasse sehr "nervös" ist. Dieser Umstand spricht also eher für als gegen diese Auffassung.

Eine Stütze für die Richtigkeit dieser Vorstellung geben uns die Versuche Klose's ${ }^{1}$ ). Dieser Experimentator beobachtete bei seinen Hunden nach Eingabe ganz kleiner Dosen von Jod (als Jodalkali), Pulsbeschleunigung neben den anderen Symptomen des Hyperthyeroidismus. Das Zustandekommen derselben ist so zu erklären, dass Jod einen vermehrten Übergang von Jodthyreoglobulin aus der Thyreoidea in den Kreislauf bewirkt. Alle Hunde reagieren nun in dieser Weise nicht auf Jodeingabe, denn andere Experimentatoren konnten die Versuche Klose's nicht bestätigen. Das rührt daher, dass Klose, wie er selbst angibt, durch Inzucht stark degenerierte Foxterriers verwendete, während seine Nachprüfer andere Hundesorten gebrauchten. Eine bessere Stütze für das Zustandekommen des Hyperthyreoidismus kann man sich wohl nicht denken. Klose's Versuche sind das vollkommene Analogon zu den Fällen von Hyperthyreoidismus durch Jodgebrauch (Jodhyperthyreoidismus) beim Menschen ${ }^{2}$ ).

Es liegt nun nichts der Erklärung im Wege, dass die Hunde Heinatz' auch Tiere mit leicht ansprechendem Nervensystem waren. Leider ist aus dem oben angeführten Passus seiner Arbeit nichts über die Rassen angegeben.

Genau das gleiche ist von den Hunden Livon's und Patta's ${ }^{3}$ ) zu sagen, welche beide bei ihren Tieren mitunter Pulsbeschleunigung und Blutdruckerhöhung beobachtet haben (vgl. Abschnitt 3). Es muss in Zukunft mehr auf die Rasse geachtet werden.

1) H. Klose, A. Lampé und R. Lisegang, Die Basedow'sche Krankheit. Eine chirurgisch - experimentelle und biologische Studie. Beitr. z. klin. Chirurgie Bd. 77 S. 601. 1912.

2) Siehe weiter oben.

3) loc. cit. 
Auf die Verwendung verschieden empfindlicher Tiere dürften die am Eingang dieser Mitteilung erwähnten Differenzen in den Angaben der Autoren über den Einfluss der Schilddrüse zurückzuführen sein. Es erklären sich danach auf einfache Weise die vielfachen Widersprüche, welche die experimentelle Schilddrüsenlitteratur so lange verwirrt haben.

Die starke Emptindlichkeit der einen (Menschen und Tiere) und die Nichtempfindlichkeit der anderen beruht darauf, dass ein normales, stabiles Nervensystem ungestraft eine geringe Erhöhung seines Tonus erfahren kann, nicht aber ein labiles. Ein solches wird bei Erhöhung seines Tonus schon auf Reize reagieren, welche bei einem gesunden Nervensystem noch keinen Effekt baben.

Die in dieser Abhandlung niedergelegten Beobachtungen geben uns, wie sich aus dem soeben Gesagten bereits ersehen lässt, wertvolle Aufschlusse über die Pathologie der Schilddrüse. Sie erklären uns viele klinische Symptome, die mit einem Plus und einem Minus der funktionellen Tätigkeit der Thyreoidea einhergehen. Wir begreifen nunmehr die verlangsamte Herztätigkeit der Myxödematösen und Kretinen, ihre verminderte Reaktionsfähigkeit auf Reize sowohl im Gebiete des Kreislaufs, um die es sich ja hier in erster Linie handelt, wie überhaupt im Bereich der gesamten zerebrospinalen (und auch Gemüts-) Sphäre. Wir begreifen das Zurückgehen dieser Symptome nach Thyreoidalbehandlung. Auf der anderen Seite wird uns das klinische Bild des Hyperthyreoidismus und des Basedow in vielfacher Beziehung klar. Wir verstehen die vermehrte Ansprechbarkeit des vaskulären Nervenapparates - wie überhaupt nach dem Gesagten des gesamten Nervensystems - die grosse Labilität in dessen Bereich, die Tachykardie, das Herzklopfen, das Auftreten von Aktionspulsen, den Dermographismus, um nur die vaskulären Symptome hervorzuheben. Ich will auf die Schilderung dieser Erscheinungen nicht näher eingehen, ich habe sie andernorts ${ }^{1}$ ) ausfühlich dargelegt und begründet und verweise denjenigen, der sich näher dafür interessiert, auf die dortigen Auseinandersetzungen. Nur das sei hervorgehoben, dass die Eigenschaften des Jodthyreoglobulins das Auftreten dieser Symptome unmittelbar wohl erklärt, dass jedoch ein vermehrter Jodthyreoglobulingehalt der Körpersäfte

1) loc. cit. 
582 A. Oswald: Über die Wirkung der Schilddrüse aưf den Blutkreislauf.

(also eine vermehrte Tätigkeit der Thyreoidea) nicht etwa einen ausreichenden Grund für das Auftreten von Hyperthyreoidismus bzw. Basedow abgibt, sondern dass, wie sich das aus dem Gesagten bereits ergibt, dafür ein anderer wesentlicher Faktor in Betracht kommen muss: eine gesteigerte Ansprechbarkeit des Nervensystems.

\section{Schlusssätze.}

Ich fasse die Hauptergebnisse dieser Untersuchungen in folgenden Schlusssätzen zusammen :

1. Jodthyreoglobulin erhöht in ausgesprochener Weise die Ansprechbarkeit der Vagusendigungen, des Depressors und des Splanchnicus gegenüber dem faradischen Strome.

2. Es verstärkt den hämodynamischen Adrenalineffekt, was sowohl die Höhe wie die Dauer desselben anbelangt.

3. Diese Eigenschaften steigern sich mit dem Jodgehalt der Präparate.

4. Jod allein ist jedoch nicht maassgebend, da ionisiertes Jod, Jodkasein, Jodtyrosin diese Eigenschaften nicht besitzen.

5. Das Baumann sche Jodothyrin besitzt die gleichen Eigenschaften wie das Jodthyreoglobulin, jedoch in vermindertem Maasse.

6. Jodthyreoglobulin aus Kröpfen hat die gleichen physiologischen Eigenschaften wie das aus gesunden Schilddrüsen, jedoch bei geringerem Jodgehalt in entsprechend geringerem Grade.

7. Jodthyreoglobulin aus Basedowkröpfen verhält sich wie das aus anderen Kröpfen bzw. aus gesunden Schilddrüsen.

8. Das Jodthyreoglobulin besitzt die Eigenschaft, den Tonus des animalen und vegetativen Nervensystems in exquisiter Weise zu erhöhen.

9. Die physiologischen Eigenschaften des Jodthyreoglobulins erklären die Mehrzahl der klinischen Symtome sowohl der Hypowie der Hyperthyreosen, (Myxödem, Hyperthyreoidismus, Basedow). 
$\frac{\bar{\Phi}}{\mathscr{E}}$
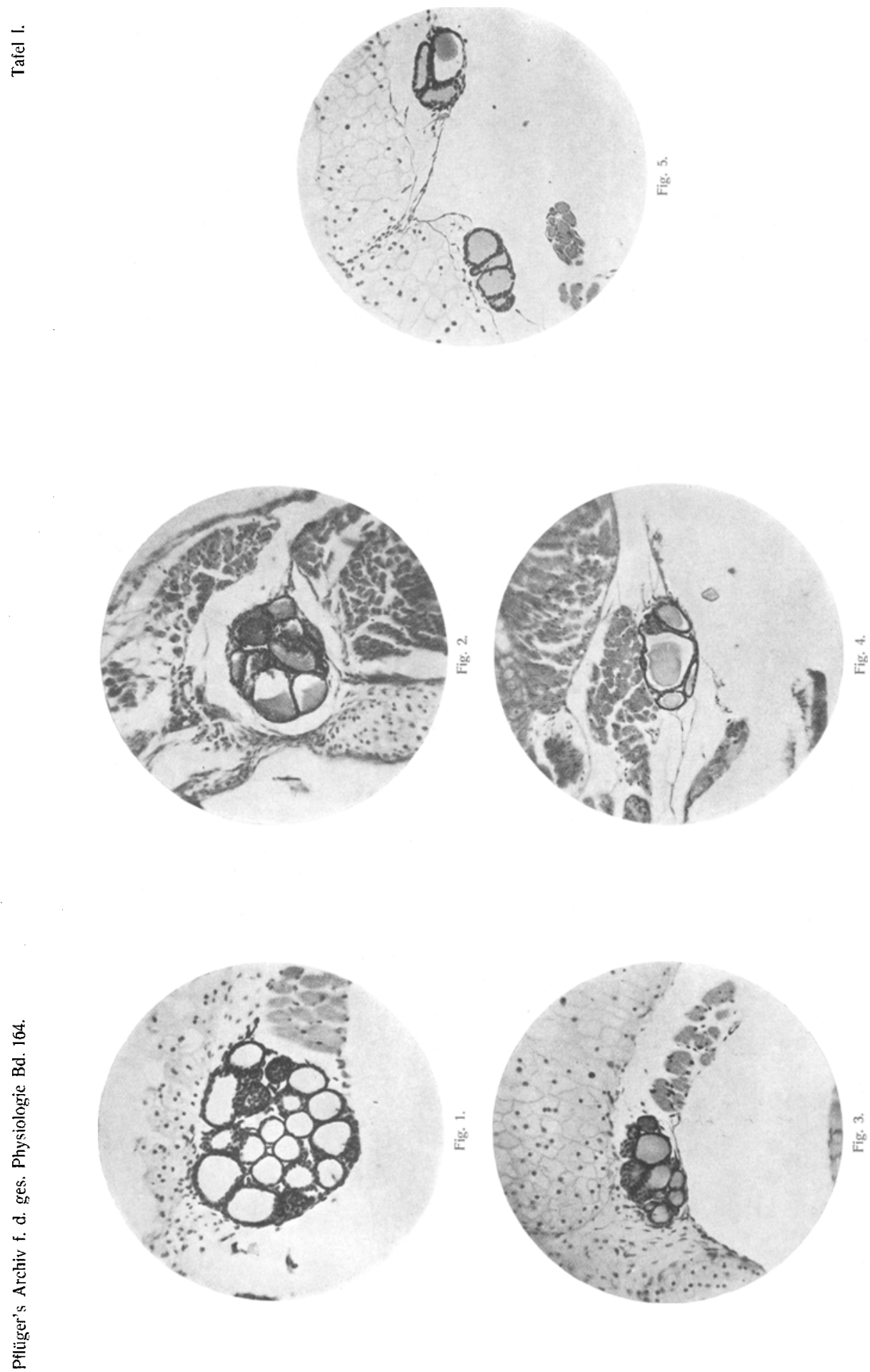

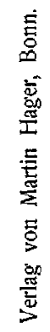



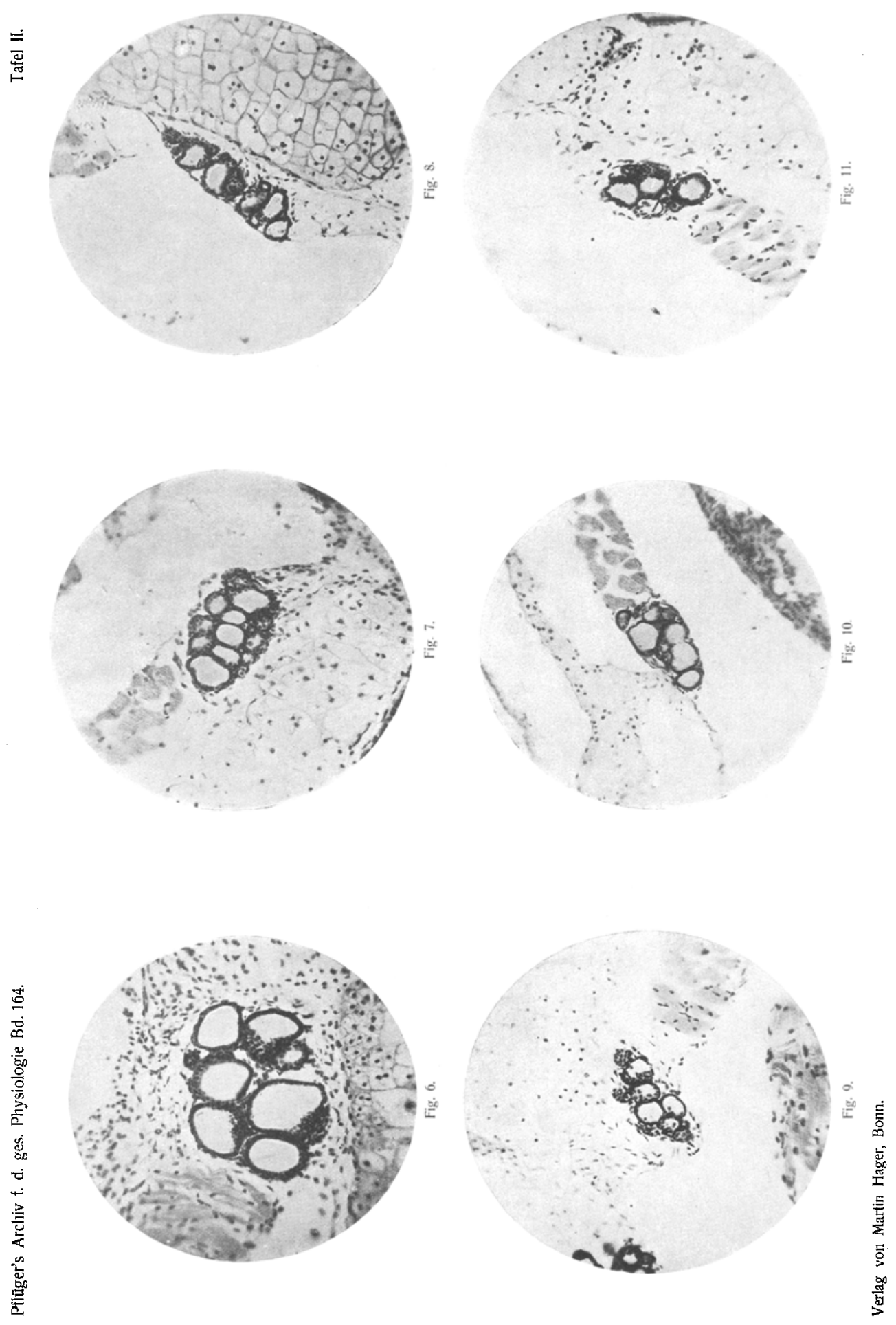


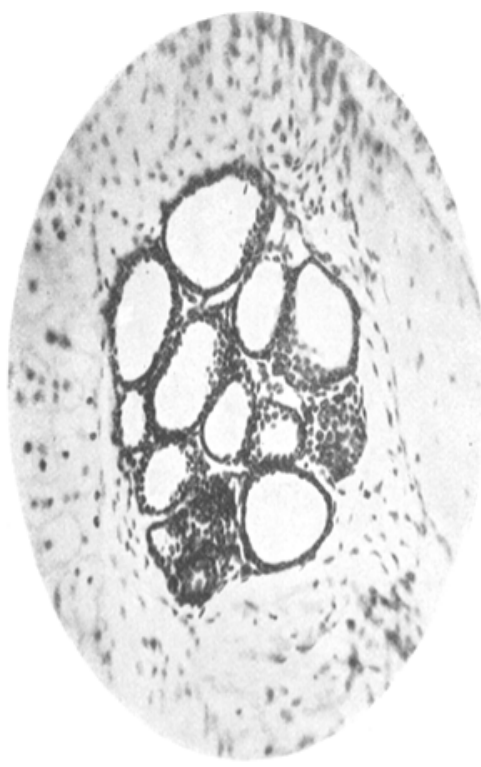

Fig. 12.

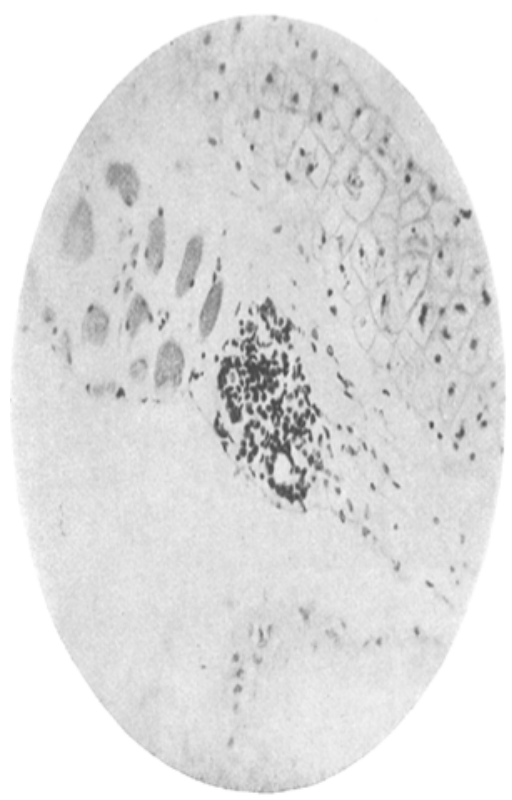

Fig. 14.

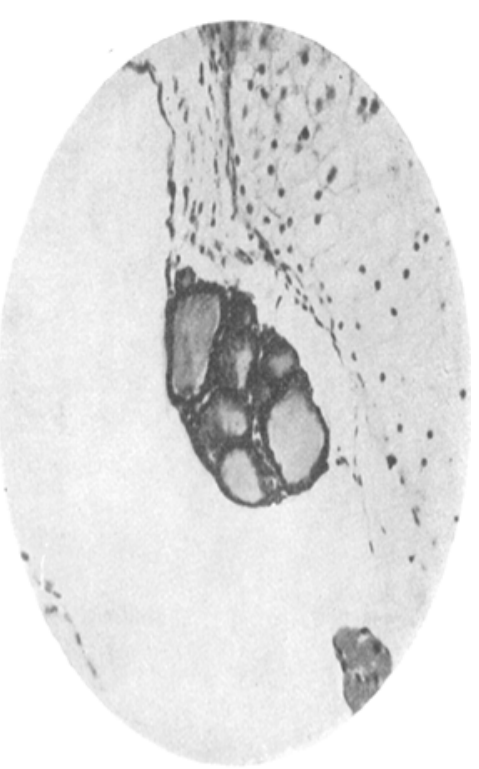

Fig. 13.

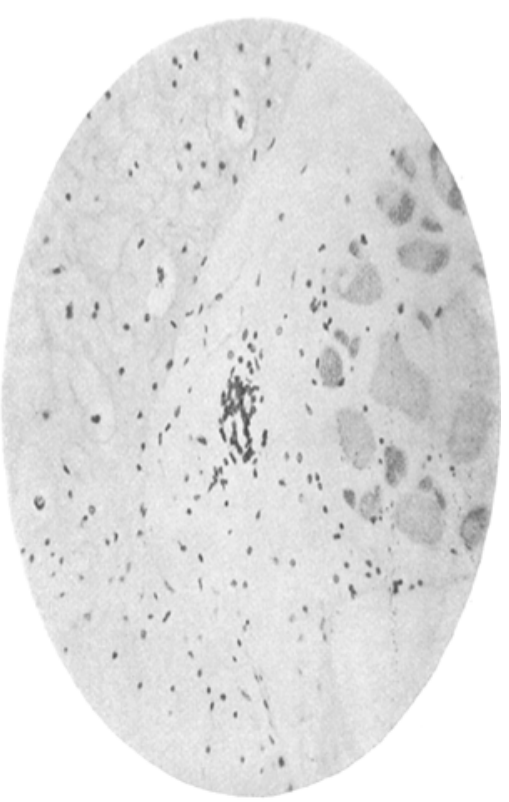

Fig. 15.

Verlag von Martin Hager, Bonn. 

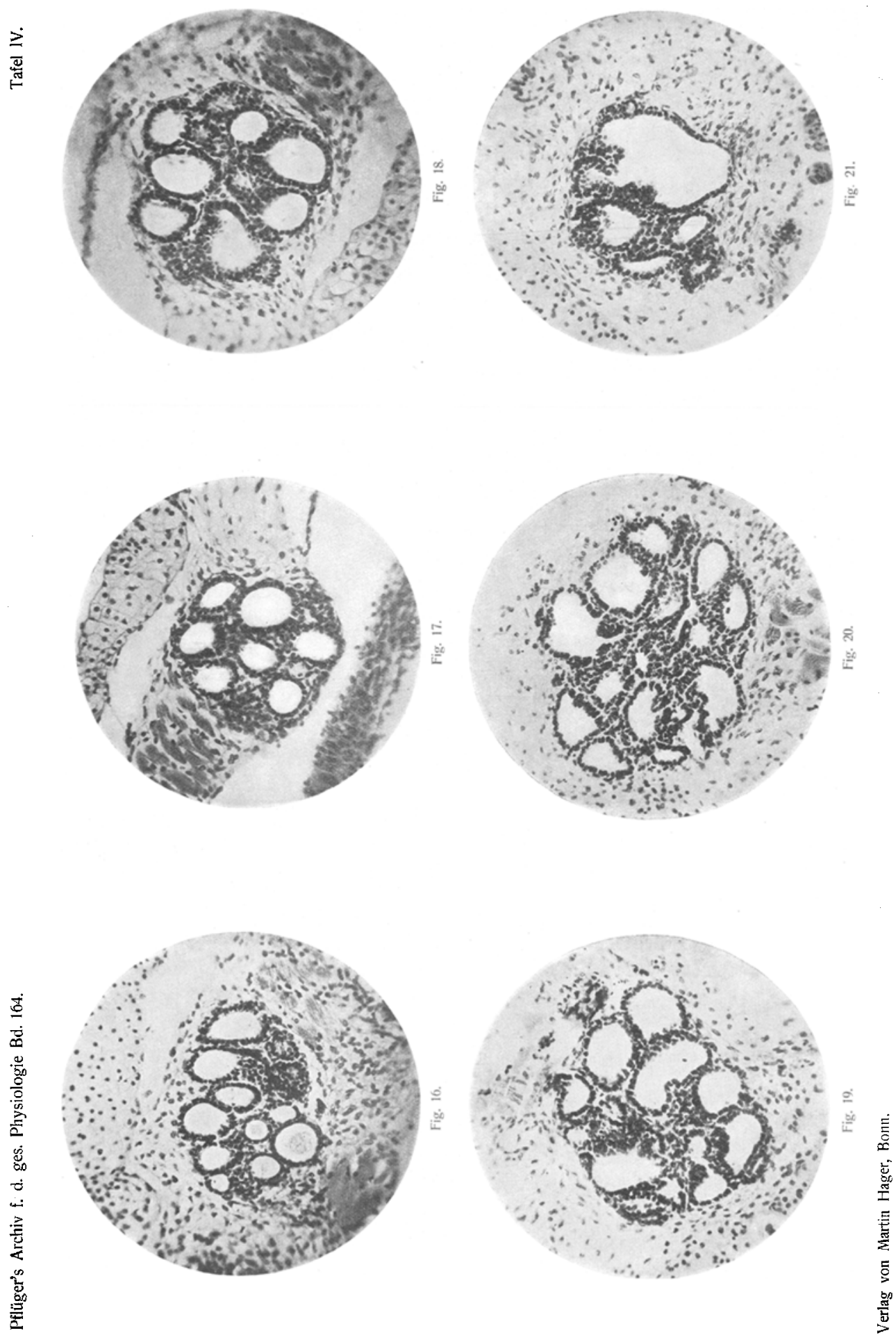
离
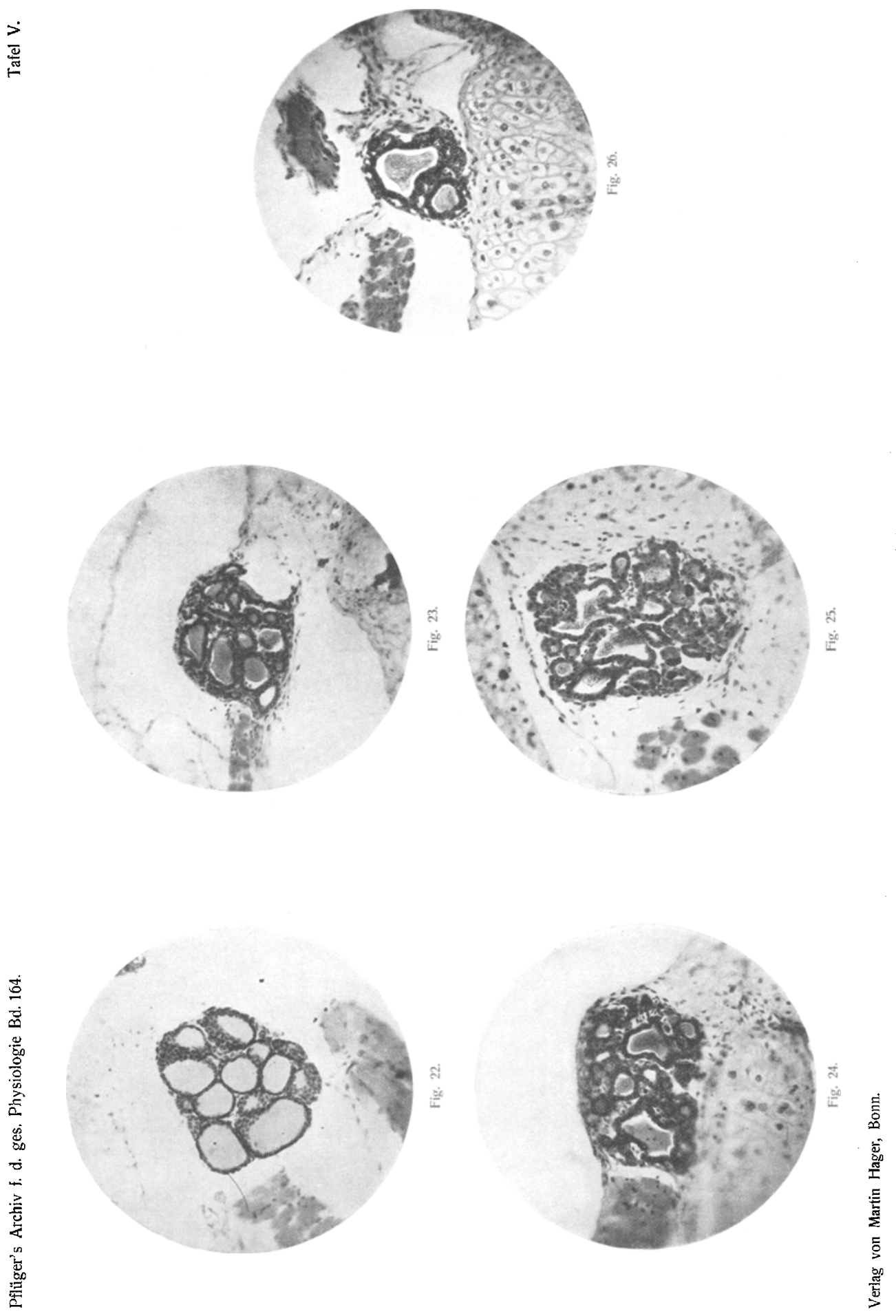

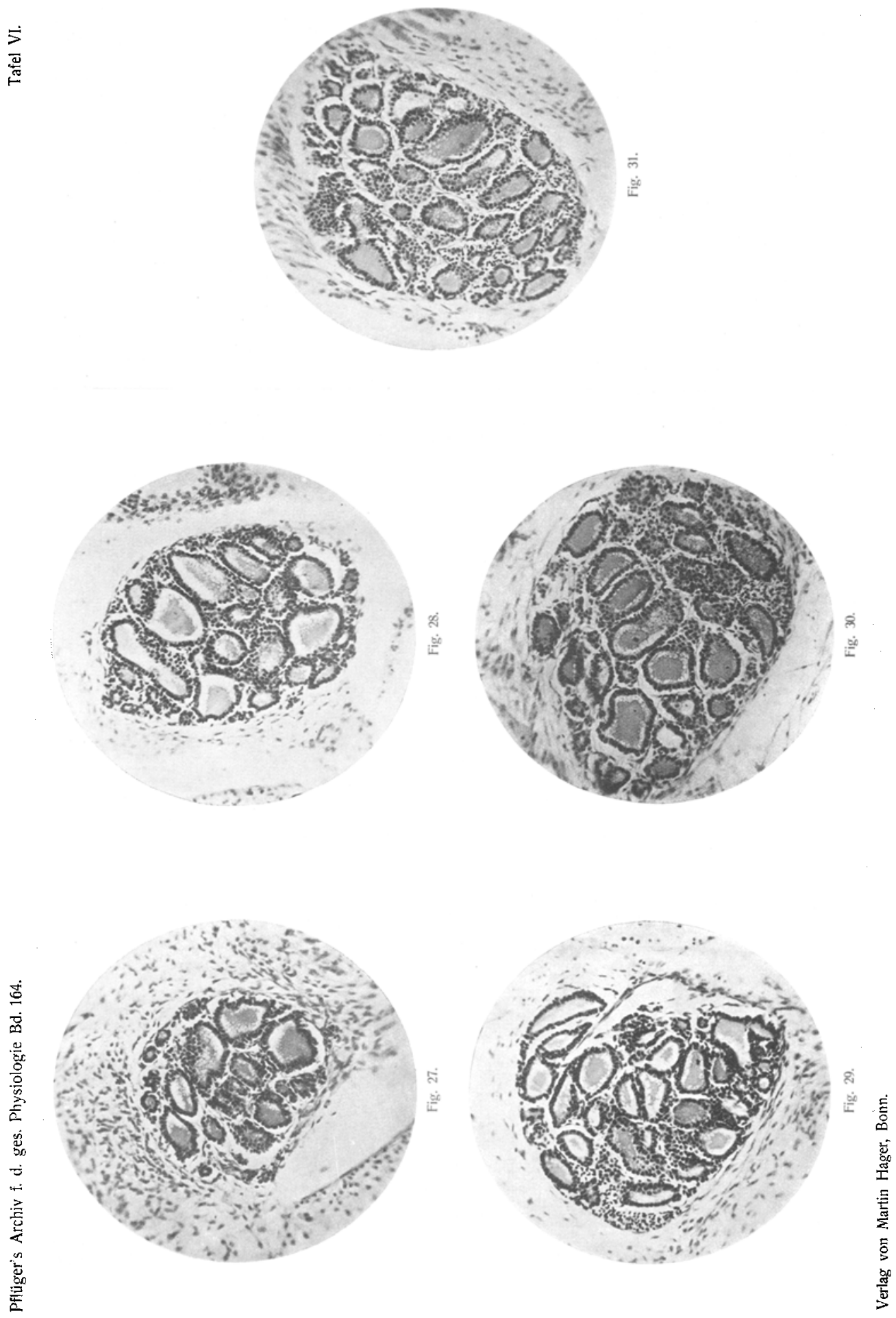
Pflüger's Archiv f. d. ges. Physiologie Bd. 164.

Tafel VII.

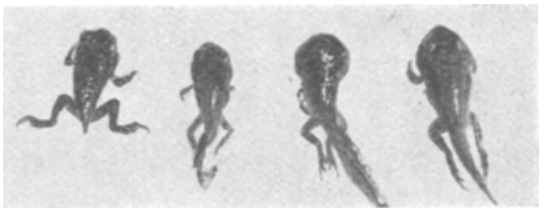

Fig. 32.

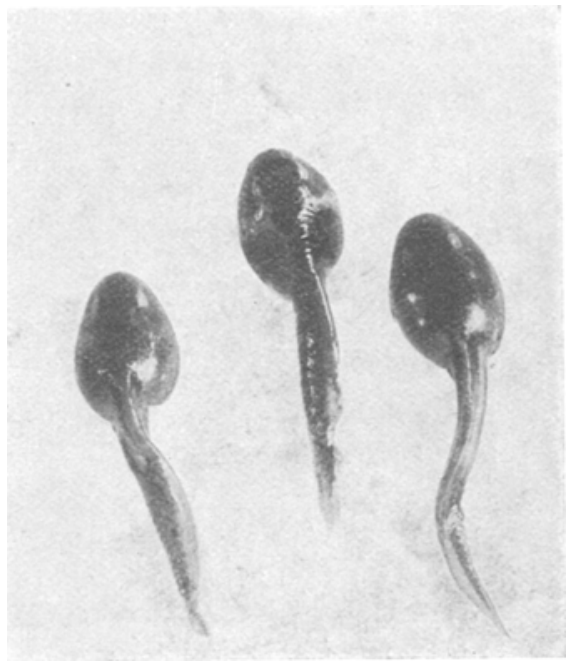

Fig. 33.

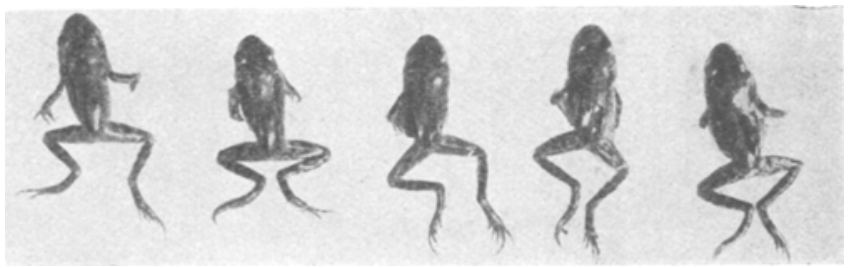

Fig. 34 .

\footnotetext{
V erlag von Martin Hager, Bonn.
} 


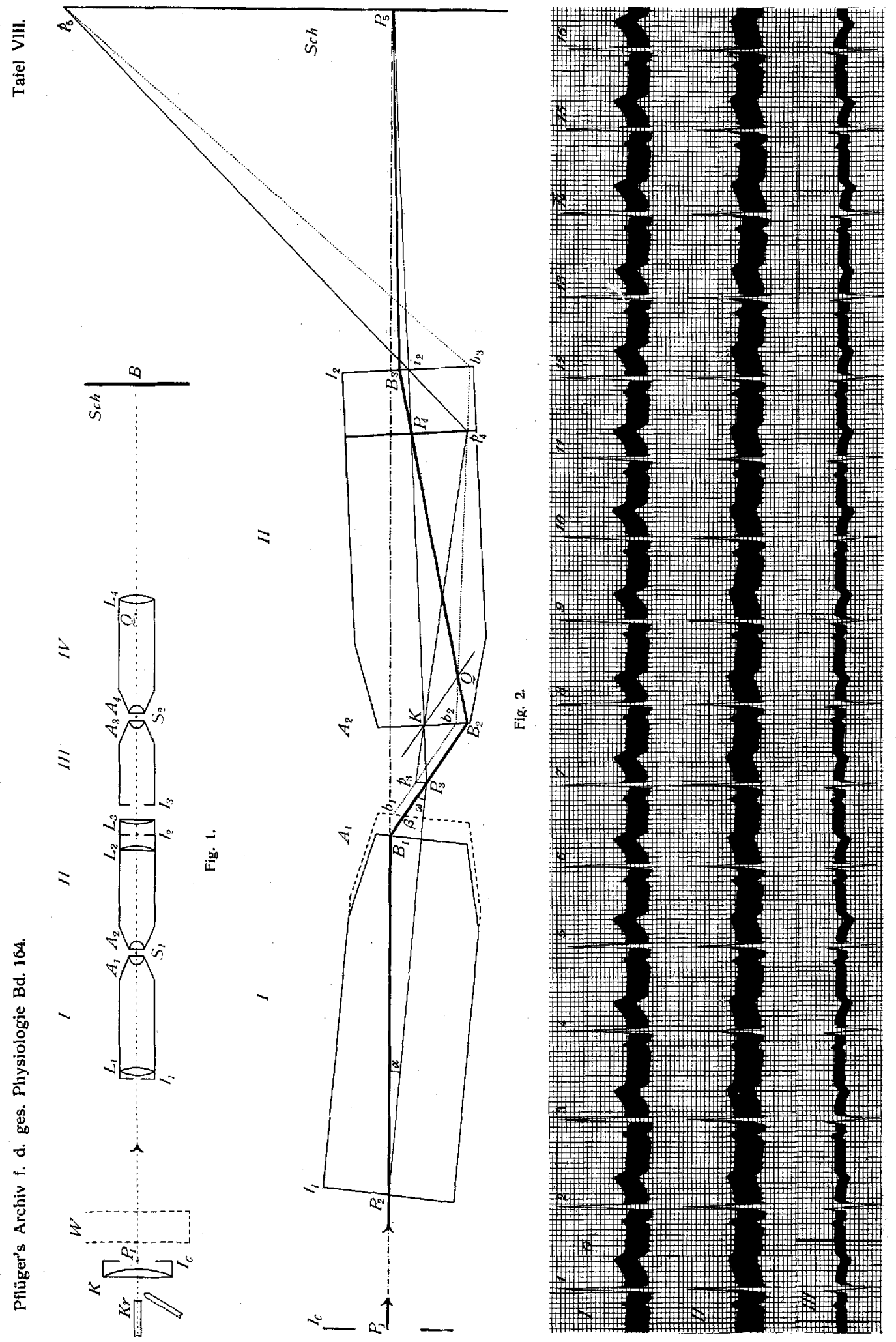

i 



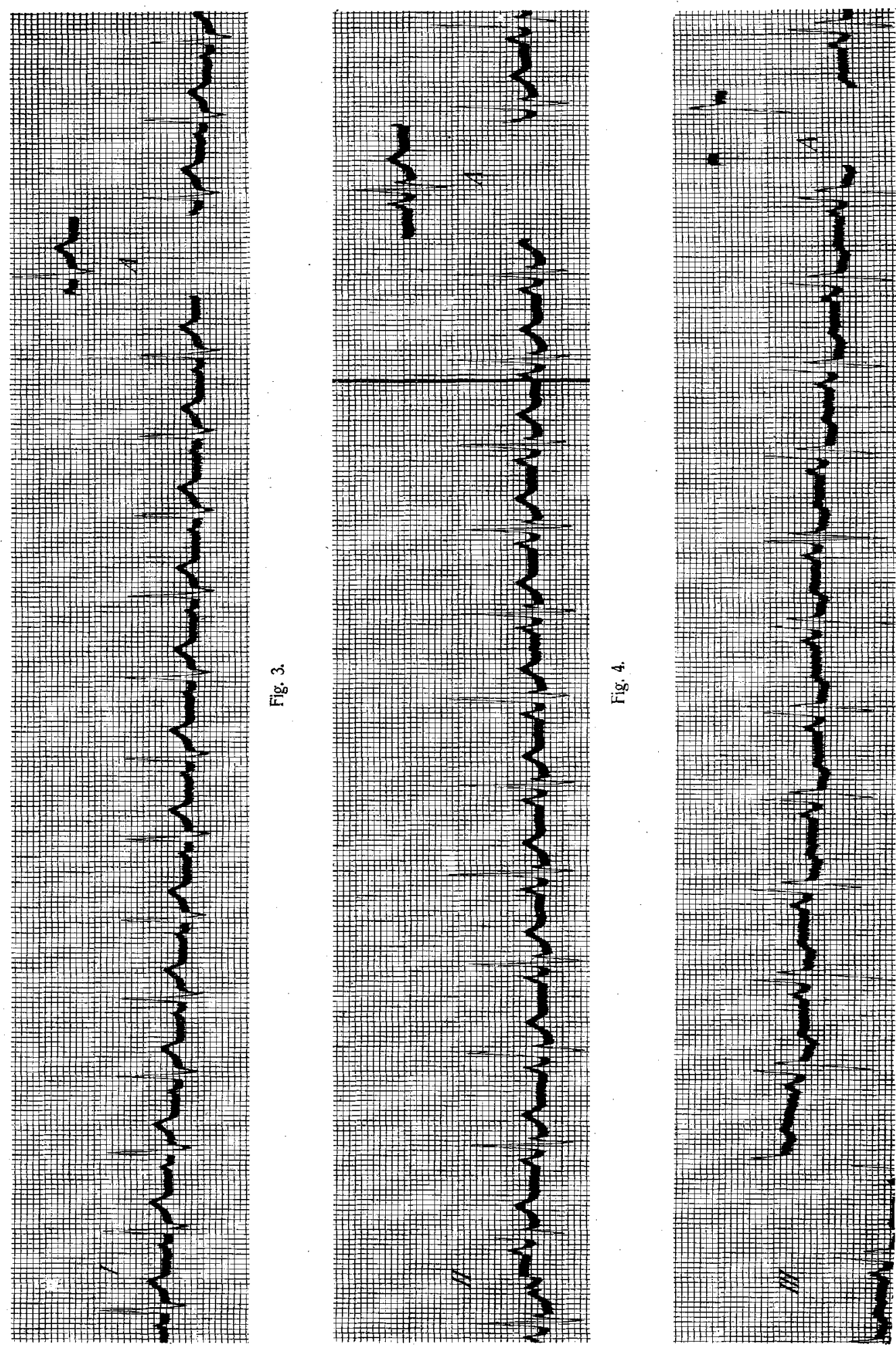

(

¿11

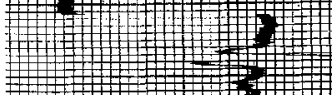

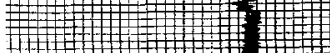

¿

¿

¿

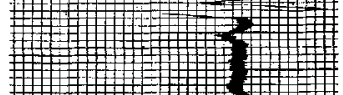

弗

¿

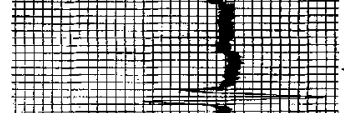

1111
1

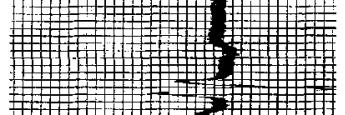

$\rightarrow$

is

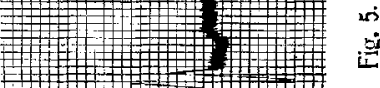

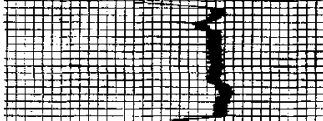

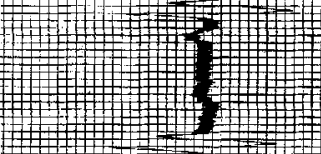

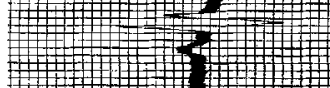

\begin{tabular}{l}
$\square$ \\
\hline
\end{tabular}

¿

(

¿ \#

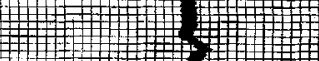

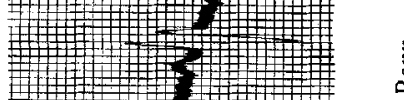

巨ั

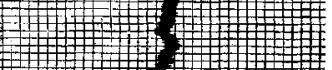

¿

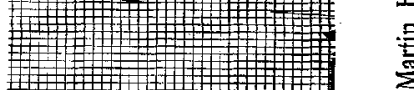

(1)

进肃

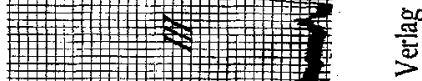

¿

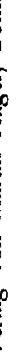



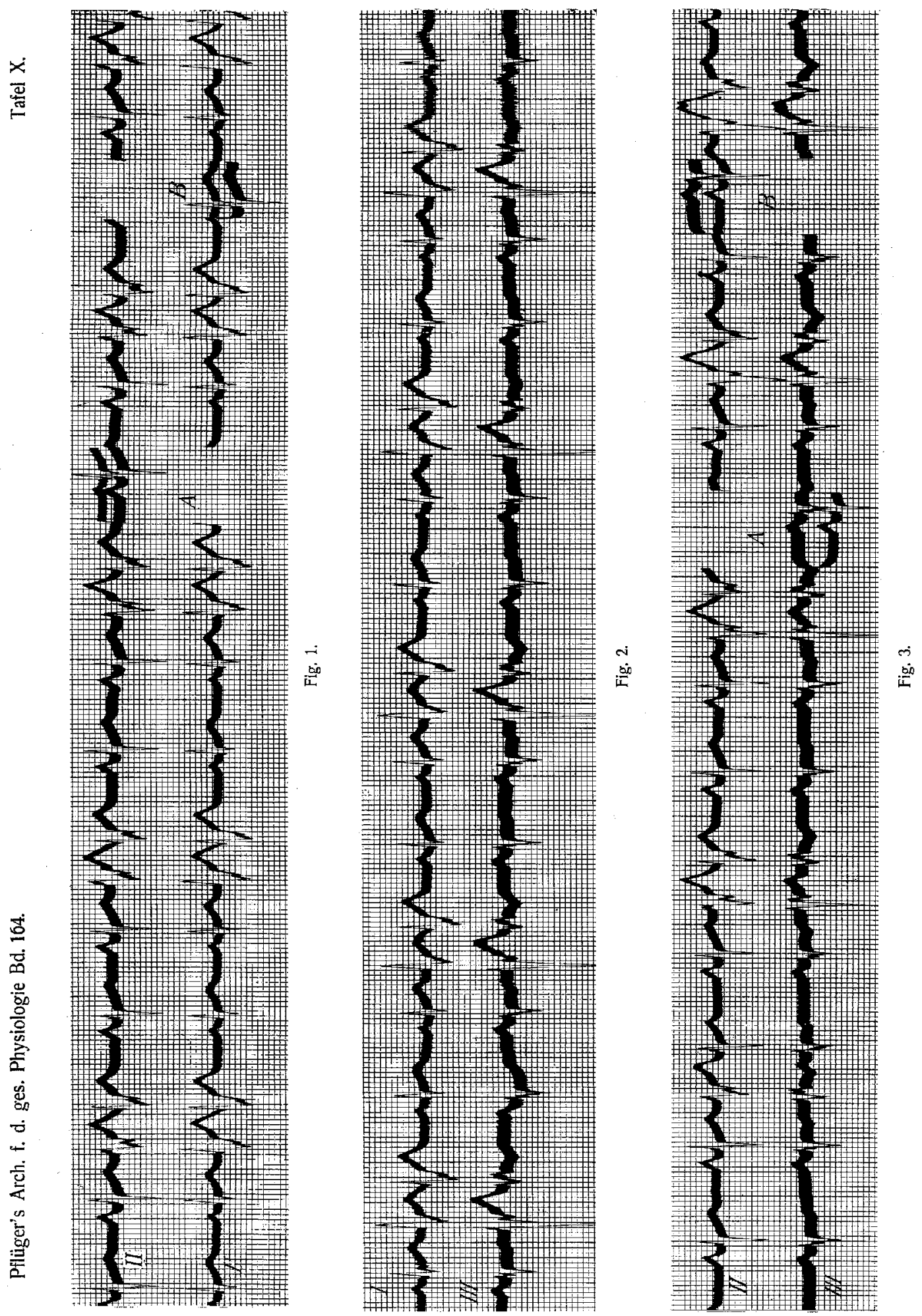


$x$
4
4

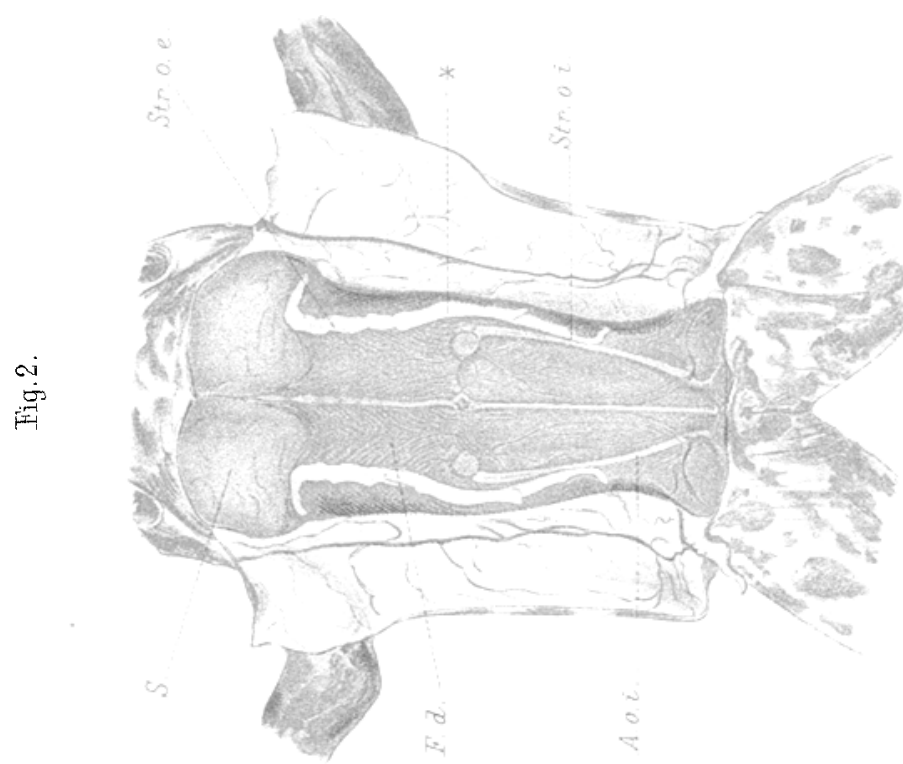

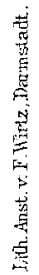

苻

夏

.

马

贾

至

离

D

4

प्र

密
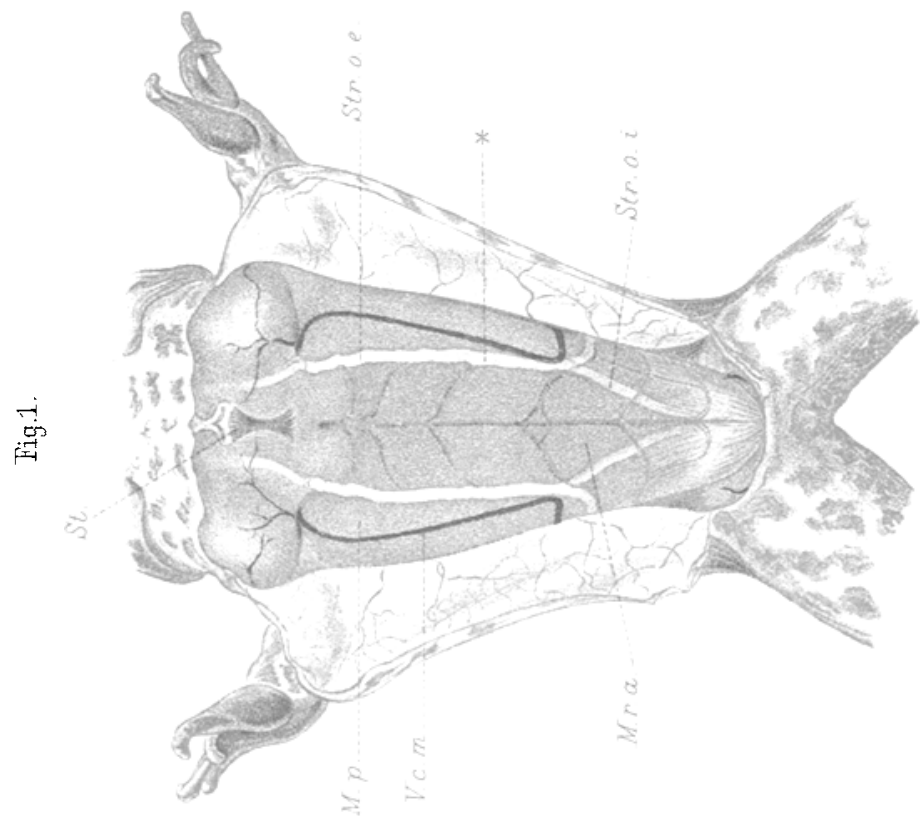